Research paper

\title{
Synthesis of ticagrelor analogues belonging to 1,2,3-triazolo[4,5-d] pyrimidines and study of their antiplatelet and antibacterial activity
}

\author{
Eric Goffin ${ }^{\mathrm{a}, 1}$, Nicolas Jacques ${ }^{\mathrm{b}, 1}$, Lucia Musumeci $^{\mathrm{b}}$, Alain Nchimi ${ }^{\mathrm{b}}$, Cécile Oury ${ }^{\mathrm{b}}$, \\ Patrizio Lancellotti ${ }^{b}$, Bernard Pirotte ${ }^{\text {a, * }}$ \\ a Laboratory of Medicinal Chemistry, Center for Interdisciplinary Research on Medicines (CIRM), University of Liege, CHU Sart Tilman, Liege, Belgium \\ ${ }^{\mathrm{b}}$ Laboratory of Cardiology, GIGA Cardiovascular Sciences, University of Liege, Department of Cardiology, University of Liege Hospital, CHU Sart Tilman, \\ Liege, Belgium
}

\section{A R T I C L E I N F O}

Article history:

Received 27 May 2020

Received in revised form

13 August 2020

Accepted 15 August 2020

Available online 23 August 2020

\section{Keywords:}

Ticagrelor

Antiplatelet agents

Antibiotics

1,2,3-triazolo[4,5-d]pyrimidines

8-Azapurines

\begin{abstract}
A B S T R A C T
Based on the recent observation that the antiplatelet agent ticagrelor and one of its metabolite exert bactericidal activity against gram-positive bacteria, a series of 1,2,3-triazolo[4,5-d]pyrimidines structurally related to ticagrelor were synthesized and examined as putative antiplatelet and antibacterial agents. The aim was to assess the possibility of dissociating the two biological properties and to find novel 1,2,3-triazolo[4,5-d]pyrimidines expressing antiplatelet activity and devoid of in vitro antibacterial activity. The new compounds synthesized were known metabolites of ticagrelor as well as structurally simplified analogues. Some of them were found to express antiplatelet activity and to lose the antibacterial activity, supporting the view that the two activities were not necessarily linked.
\end{abstract}

() 2020 Elsevier Masson SAS. All rights reserved.

\section{Introduction}

Ticagrelor (1; Fig. 1) is an orally active antiplatelet drug belonging to 1,2,3-triazolo[4,5-d]pyrimidines (8-azapurines), which acts by reversibly inhibiting the platelet P2Y12 receptor for ADP in a noncompetitive manner [1,2]. This drug used in human medicine was developed to improve the efficacy and circumvent the limitations of the first-generation P2Y12 inhibitors [3,4]. Although ticagrelor doesn't need to be metabolized to be active in vivo, this drug is known to be extensively metabolized via cytochrome P450 3A4 and 3A5 to form a main metabolite known as AR-C124910, representing approximately one third of ticagrelor in the circulation [5]. Ticagrelor and its main metabolite inhibit P2Y12 receptors with equivalent potency [5]. Recently, we observed that ticagrelor and its active metabolite AR-124910 also exert in vitro bactericidal activity against Gram-positive strains, including antibiotic-resistant strains, such as Staphylococcus aureus, Staphylococcus epidermidis and Enterococcus faecalis [6]. As a result, ticagrelor might prove superior to other P2Y12 inhibitors in patients

\footnotetext{
* Corresponding author.

E-mail address: b.pirotte@uliege.be (B. Pirotte).

1 These authors (E.G., N.J.) contributed equally.
}

with cardiovascular disease at risk for gram-positive bacterial infections such as infective endocarditis [6]. Another well-known additional benefit of ticagrelor compared to other clinically used P2Y12 inhibitors is also its ability to exert anti-inflammatory activity, notably by decreasing inflammatory cytokines such as interleukin 6 and tumor necrosis factor alpha as well as plateletleukocyte aggregates or by acting directly on endothelial cells, independently of the P2Y12 receptor [7-11].

$N^{7}$-substituted 7-amino-5-alkylsulfanyl-1,2,3-triazolo[4,5-d]pyrimidines, among which the antiplatelet agent ticagrelor, are relatively poorly described in the literature. Besides examples of P2Y12 receptor antagonists structurally related to ticagrelor [1,12], recent publications also reported compounds of this chemical class expressing antiproliferative activity [i.e. compound 2 [13]; compound 3 [14]; compound 4 [15]; compound 5 [16]; i.e. compound $\mathbf{6}$, identified as a lysine specific demethylase 1 (LSD1) inhibitor [17]; Fig. 1]. No indication of antibacterial activity was reported for compounds of this class until the recent discovery of the antibiotic effect of ticagrelor on several Gram-positive bacterial strains.

The present work aims at establishing structure-activity relationships in order to identify the structural elements responsible, on the one hand, for the antiplatelet activity, and, on the other hand, for the antibacterial activity of ticagrelor analogues i.e. some 


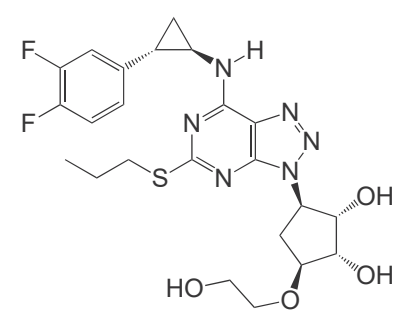

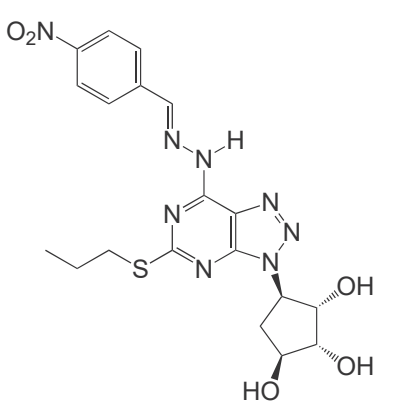

2

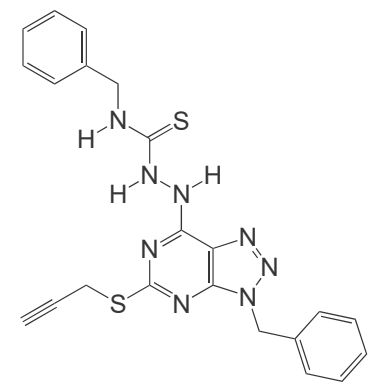<smiles>C/C(=N\Nc1nc(SCc2ccccc2)nc2c1nnn2Cc1ccccc1)c1ccccc1O</smiles>

3

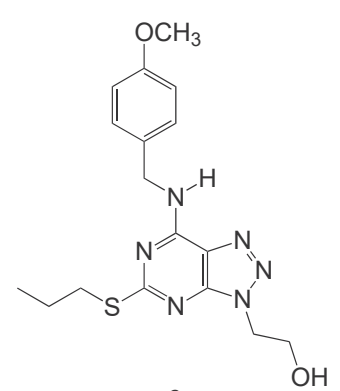

Fig. 1. Examples of $N^{7}$-substituted 7-amino-5-alkylsulfanyl-1,2,3-triazolo[4,5-d]pyrimidines described in the literature (1: ticagrelor).

of its metabolites as well as simplified structures (general formula, see 7; Fig. 2).

\section{Results and discussion}

\subsection{Chemistry}

The synthesis of the target compounds (7; Fig. 2) is described in Scheme 1. Several steps were adaptations of previously described processes [12]. Starting from thiobarbituric acid (8), alkylation in aqueous alkaline medium using an appropriate alkyl halide led to the 2-alkylsulfanyl-substituted derivatives of general formula $\mathbf{9}$, which in turn were converted into the corresponding 5-nitrosubstituted derivatives $\mathbf{1 0}$ after reaction with nitric acid in glacial acetic acid. The latter compounds reacted with phosphorus oxychloride in the presence of 2,6-lutidine to provide the corresponding 4,6-dichloro-substituted compounds 11b. These intermediates $\mathbf{1 1} \mathbf{b}$, as well as the commercially available 2,4,6trichloro-substituted analogue 11a, were easily converted into the corresponding 5-amino-substituted compounds 12a and 12b after<smiles>[R]Nc1nc([X])nc2c1nnn2[R]</smiles>

7

$$
\begin{gathered}
\mathrm{X}=\mathrm{R}^{1} \mathrm{~S}: \mathbf{7} \mathbf{a - t} \\
\mathrm{X}=\mathrm{H}: \mathbf{7 u} \\
\mathrm{X}=\mathrm{Cl}: \mathbf{7 v}
\end{gathered}
$$

Fig. 2. General formula of the newly synthesized compounds. reduction in the presence of iron powder in a mixture of acetic acid and methanol. The appropriate primary amine $\left[\mathrm{R}^{3}-\mathrm{NH}_{2}\right.$ : methylamine, ethylamine, cyclopentylamine, $(3 \mathrm{a} R, 4 S, 6 R, 6 \mathrm{a} S)-6$-amino2,2-dimethyltetrahydrocyclopenta[d][1,3]dioxol-4-ol and 2(((3aR,4S,6R,6aS)-6-amino-2,2-dimethyltetrahydro-3aH-cyclopenta[d][1,3]dioxol-4-yl)oxy)ethanol] was introduced at the 3position of intermediates 12a-b after nucleophilic substitution in methanol of one chlorine atom linked to the ortho position of the amine function. Ring closure reaction occurred after diazotization in the presence of sodium nitrite and acetic acid to provide the triazolo[4,5-d]pyrimidine intermediates 14a-c. Due to the increased sensitivity of the 7-position of triazolo[4,5- $d$ ]pyrimidines to nucleophilic substitution as a probable result of the vicinity of the triazole ring, the chlorine atom at this position was easily substituted with the appropriate alkyl/aralkylamine $\left[\mathrm{R}^{2}-\mathrm{NH}_{2}\right.$; cyclopropylamine, 3,4-difluorophenethylamine, $\quad(1 R, 2 S)-2-$ phenylcyclopropylamine or $(1 R, 2 S)-2-(3,4$-difluorophenyl)cyclopropylamine] in acetonitrile in the presence of triethylamine, leading to intermediates 15a-c. Strong nucleophiles such as thiols were able to displace the last chlorine atom linked at the 5-position of the triazolo[4,5-d]pyrimidine ring system giving access to compounds of general formula 7. The final compounds bearing a polyhydroxypentane group (compounds $\mathbf{7 h - t}$ ) at the 3-position were obtained after removal of the acetonide protecting group under strong acidic conditions.

An interesting observation was made with the target compounds bearing an aralkylamino group at the 7-position of the 1,2,3-triazolo[4,5-d]pyrimidine core structure. The ${ }^{1} \mathrm{H}$ NMR spectral data of these drugs recorded in DMSO- $d_{6}$ always revealed the presence of two groups of signals. This was particularly evident for the $\mathrm{N}-\mathrm{H}$ proton linked at the 7-position of the triazolopyrimidine ring and for the $\mathrm{C}-\mathrm{H}$ proton linked at the first carbon atom of the cyclopropyl ring of (3,4-difluorophenyl)cyclopropylaminosubstituted compounds (Table 2). Such an observation is in accordance with the existence of an equilibrium between two 
<smiles>Oc1cc(O)nc(S)n1</smiles><smiles>C1CC2CCCC2C1</smiles><smiles>[R1]Sc1nc(O)cc(O)n1</smiles><smiles>C1=CC=C1</smiles>
9

9.1: $\mathrm{R}^{1}=\mathrm{CH}_{3}$ 9.2: $\mathrm{R}^{1}=\mathrm{CH}_{2} \mathrm{CH}_{3}$<smiles>[R]Sc1nc(O)c([N+](=O)[O-])c(O)n1</smiles>

10

10.1: $\mathrm{R}^{1}=\mathrm{CH}_{3}$ 10.2: $\mathrm{R}^{1}=\mathrm{CH}_{2} \mathrm{CH}_{3}$<smiles>C1=CCCC1</smiles><smiles>[X]c1nc(Cl)c([N+](=O)[O-])c(Cl)n1</smiles>

11

11a: $\mathrm{X}=\mathrm{Cl}$

11b.1: $\mathrm{X}=\mathrm{CH}_{3} \mathrm{~S}$

11b.2: $\mathrm{X}=\mathrm{CH}_{3} \mathrm{CH}_{2} \mathrm{~S}$<smiles>C1C[Te]C1</smiles><smiles>[R]Nc1nc([X])nc(Cl)c1N</smiles>

13

13a.1-2: $\mathrm{X}=\mathrm{Cl}$

13b.1-9: $X=R^{1} S$

13c.1: $X=H$<smiles>[X]c1nc(Cl)c(N)c(Cl)n1</smiles>

12

12a: $\mathrm{X}=\mathrm{Cl}$

12b.1: $\mathrm{X}=\mathrm{CH}_{3} \mathrm{~S}$

12b.2: $X=\mathrm{CH}_{3} \mathrm{CH}_{2} \mathrm{~S}$

12b.3: $\mathrm{X}=\mathrm{CH}_{3} \mathrm{CH}_{2} \mathrm{CH}_{2} \mathrm{~S}$

12c: $X=H$<smiles>[R]Nc1nc(Cl)nc2c1nnn2[R]</smiles>

15a.1

15a.3<smiles>[R]Nc1nc(S[R])nc2c1nnn2[R]</smiles>

$16 \mathrm{~h}-\mathrm{n}$<smiles>[R]Nc1nc([X])nc2c1nnn2[C@@H]1CC([R20])[C@H]2OC(C)(C)O[C@H]21</smiles>

16a-n: $X=R^{1} S$

15a.2: $X=\mathrm{Cl}$

15c.1: $X=H$

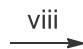<smiles>[R]Nc1nc([X])nc2c1nnn2[C@@H]1C[C@H](O[R20])[C@@H](O)[C@H]1O</smiles>

7h-v

Scheme 1. Synthesis of Ticagrelor Analogues Belonging to 1,2,3-Triazolo[4,5-d]pyrimidines

Reagents and conditions: (i) $\mathrm{R}^{1} \mathrm{X}, \mathrm{KOH}$, water, $80^{\circ} \mathrm{C}$, sealed tube, $2 \mathrm{~h}$; (ii) nitric acid, acetic acid, $0{ }^{\circ} \mathrm{C}$ to r.t., $1 \mathrm{~h}$; (iii) $\mathrm{POCl}, 2,6$-lutidine, $0{ }^{\circ} \mathrm{C}-80{ }^{\circ} \mathrm{C}, 2 \mathrm{~h}$; (iv) iron powder, acetic acid, methanol, r.t., $2 \mathrm{~h}$; (v) $\mathrm{R}^{3} \mathrm{NH}_{2}$, methanol, $110^{\circ} \mathrm{C}$, sealed tube, $1 \mathrm{~h}$; (vi) $\mathrm{NaNO}_{2}$, acetic acid, $0{ }^{\circ} \mathrm{C}$ to r.t., $2 \mathrm{~h}$; (vii) $\mathrm{R}^{2} \mathrm{NH}_{2}, \mathrm{TEA}$, acetonitrile, $80{ }^{\circ} \mathrm{C}, 1-4 \mathrm{~h}$; (viii) $\mathrm{R}^{1} \mathrm{SH}$, $\mathrm{K}_{2} \mathrm{CO}{ }_{3}$, acetonitrile, $60-110^{\circ} \mathrm{C}, 3 \mathrm{~h}$; (ix) $\mathrm{HCl}$, methanol, r.t., $30 \mathrm{~min}$.

conformational isomers resulting from the rotation around the $\mathrm{C}^{8}$ $\mathrm{N}^{1}$ bond (Fig. 3) (also reported for azaadenine derivatives [18]), the former isomers being the two preferred conformations assuming an optimal delocalization of electrons in the planar "amidine" system (numbering $\mathrm{N}^{7}-\mathrm{C}^{8}-\mathrm{N}^{1}$ in Fig. 3). In accordance with the planarity of the system, and for steric reasons, conformer $\mathrm{B}$ is expected to be the major form (estimation from NMR data in DMSO- $d_{6}$ at r.t.: $80 \%$ of conformer B versus $20 \%$ of conformer $A$ ). The preference of the $\mathrm{B}$ conformation for ticagrelor (and for related analogues) is confirmed by crystallographic data obtained with the antiplatelet agent (alone or in co-crystalisation) revealing the presence of conformer $B$ in the crystal lattices [19-21]. The planarity of the "amidine" system was also confirmed from the crystallographic data obtained with ticagrelor alone providing a torsion angle $\mathrm{C}^{9}-\mathrm{C}^{8}-\mathrm{N}^{1}-\mathrm{C}^{1}$ of $178.6^{\circ}$, thus not far fom the planarity $\left(180.0^{\circ}\right)[20]$.

The attribution of the chemical shifts reported in Table 2 is deduced from the influence of the proximity of the $\mathrm{N}^{1}$ nitrogen atom, which is expected to induce a greater deshielding of the $\mathrm{C}-\mathrm{H}$ proton in the case of conformer $\mathrm{A}$, and a greater deshielding of the $\mathrm{N}-\mathrm{H}$ proton in the case of conformer $\mathrm{B}$. 
<smiles></smiles>

\section{Conformer A}<smiles></smiles>

Conformer B

Fig. 3. Conformational isomerism resulting from the rotation around the $\mathrm{C}^{8}-\mathrm{N}^{1}$ bond of $N^{7}$-substituted 7-amino-1,2,3-triazolo[4,5-d]pyrimidines.

\subsection{Biological evaluation}

Table 1 reports the biological results (antiplatelet and antibacterial activity) obtained with ticagrelor (1), several of its metabo-

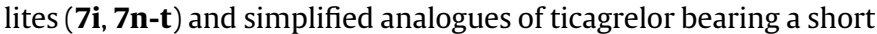
alkyl group at the 3-position and/or a short alkylsulfanyl group at the 5-position and/or a simplified aralkylamino group at the 7position (7a-h, $\mathbf{7 j}-\mathbf{m}, \mathbf{7} \mathbf{u}-\mathbf{v})$. The aim was to examine the antiplatelet versus antibacterial activity of other known ticagrelor metabolites as well as a series of original simplified analogues.

The antiplatelet activity of the tested molecules was analyzed by light transmission aggregometry upon platelet stimulation with ADP in citrated platelet-rich-plasma using a drug concentration

Table 2

${ }^{1} \mathrm{H}$ NMR Chemical Shifts (in ppm) Attributed to the $\mathrm{C}-\mathrm{H}$ Proton $\left(\mathrm{H}^{\mathrm{a}}\right.$ or $\left.\mathrm{H}^{\mathrm{a}}\right)$ Linked to the First Cyclopropyl Carbon Atom and to the $\mathrm{N}-\mathrm{H}$ Proton $\left(\mathrm{H}^{\mathrm{b}}\right.$ or $\left.\mathrm{H}^{\mathrm{b}}\right)$ at the 7-Position of the 1,2,3-Triazolo[4,5-d]pyrimidine Ring.<smiles></smiles>

Minor $(\sim 20 \%)$

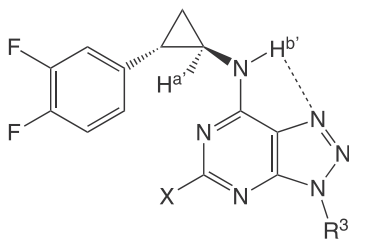

Major $(\sim 80 \%)$

\begin{tabular}{|c|c|c|c|c|c|c|}
\hline \multirow[t]{2}{*}{ Cmpd } & \multirow[t]{2}{*}{$-\mathrm{X}$} & \multirow[t]{2}{*}{$-\mathrm{R}^{3}$} & \multicolumn{2}{|l|}{$\mathrm{C}-\mathrm{H}$} & \multicolumn{2}{|l|}{$\mathrm{N}-\mathrm{H}$} \\
\hline & & & $\mathrm{H}^{\mathrm{a}}$ & $\mathrm{H}^{\mathrm{a}^{\prime}}$ & $\mathrm{H}^{\mathrm{b}}$ & $\mathrm{H}^{\mathrm{b}^{\prime}}$ \\
\hline $7 a$ & $-\mathrm{SCH}_{3}$ & $-\mathrm{CH}_{3}$ & 3.76 & 3.15 & 8.96 & 9.34 \\
\hline 7d & $-\mathrm{SCH}_{2} \mathrm{CH}_{3}$ & $-\mathrm{CH}_{2} \mathrm{CH}_{3}$ & 3.77 & 3.16 & 8.96 & 9.37 \\
\hline $7 \mathbf{i}$ & $-\mathrm{SCH}_{2} \mathrm{CH}_{2} \mathrm{CH}_{3}$ & $-\mathrm{C}_{5} \mathrm{H}_{6}(\mathrm{OH})_{3}$ & 3.78 & 3.16 & 8.93 & 9.35 \\
\hline 1 & $-\mathrm{SCH}_{2} \mathrm{CH}_{2} \mathrm{CH}_{3}$ & $-\mathrm{C}_{5} \mathrm{H}_{6}(\mathrm{OH})_{2} \mathrm{OCH}_{2} \mathrm{CH}_{2} \mathrm{OH}$ & 3.79 & 3.16 & 8.94 & 9.36 \\
\hline $7 \mathbf{u}$ & $-\mathrm{H}$ & $-\mathrm{C}_{5} \mathrm{H}_{6}(\mathrm{OH})_{3}$ & 3.82 & 3.29 & 9.04 & 9.34 \\
\hline $7 v$ & $-\mathrm{Cl}$ & $-\mathrm{C}_{5} \mathrm{H}_{6}(\mathrm{OH})_{3}$ & 3.82 & 3.14 & 9.53 & 9.81 \\
\hline
\end{tabular}

equal to ticagrelor $\mathrm{IC}_{50}(1.8 \mu \mathrm{M})$ (Fig. 4). The antibacterial activity against methicillin-resistant Staphylococcus aureus (MRSA, ATCC BAA-1556) was determined by the broth microdilution method as recommended by EUCAST guidelines.

Only ticagrelor (1) and its main metabolite AR-C124910 (7i) showed antibacterial activity against MRSA. The other compounds revealed absence of activity against bacteria up to a concentration

Table 1

Antiplatelet activity of 1,2,3-triazolo[4,5-d]pyrimidines structurally related to ticagrelor; antibacterial activity established on methicillin-resistant S. aureus.

$$
\text { (N) }
$$

\begin{tabular}{|c|c|c|c|c|c|}
\hline Cpd & $-\mathrm{X}$ & $-R^{2}$ & $-\mathrm{R}^{3}$ & Antiplatelet activity (Fold-inhibition vs vehicle ${ }^{a}$ ) & Antibacterial activity $\left(\mathrm{MIC}^{\mathrm{b}}, \mu \mathrm{M}\right)$ \\
\hline $7 a$ & $-\mathrm{SCH}_{3}$ & $-\mathrm{CH}\left(\mathrm{CH}_{2}\right) \mathrm{CH}-\mathrm{C}_{6} \mathrm{H}_{3} \mathrm{~F}_{2}$ & $-\mathrm{CH}_{3}$ & $0.94 \pm 0.09$ & $>200$ \\
\hline 7b & $-\mathrm{SCH}_{3}$ & $-\mathrm{CH}\left(\mathrm{CH}_{2}\right) \mathrm{CH}-\mathrm{C}_{6} \mathrm{H}_{3} \mathrm{~F}_{2}$ & $-\mathrm{CH}_{2} \mathrm{CH}_{3}$ & $0.87 \pm 0.04$ & $>200$ \\
\hline 7c & $-\mathrm{SCH}_{2} \mathrm{CH}_{3}$ & $-\mathrm{CH}\left(\mathrm{CH}_{2}\right) \mathrm{CH}-\mathrm{C}_{6} \mathrm{H}_{3} \mathrm{~F}_{2}$ & $-\mathrm{CH}_{3}$ & $0.97 \pm 0.08$ & $>200$ \\
\hline 7d & $-\mathrm{SCH}_{2} \mathrm{CH}_{3}$ & $-\mathrm{CH}\left(\mathrm{CH}_{2}\right) \mathrm{CH}-\mathrm{C}_{6} \mathrm{H}_{3} \mathrm{~F}_{2}$ & $-\mathrm{CH}_{2} \mathrm{CH}_{3}$ & $0.91 \pm 0.15$ & $>200$ \\
\hline 7e & $-\mathrm{SCH}_{2} \mathrm{CH}_{2} \mathrm{CH}_{3}$ & $-\mathrm{CH}\left(\mathrm{CH}_{2}\right) \mathrm{CH}-\mathrm{C}_{6} \mathrm{H}_{3} \mathrm{~F}_{2}$ & $-\mathrm{CH}_{3}$ & $1.08 \pm 0.05$ & $>200$ \\
\hline $7 f$ & $-\mathrm{SCH}_{2} \mathrm{CH}_{2} \mathrm{CH}_{3}$ & $-\mathrm{CH}\left(\mathrm{CH}_{2}\right) \mathrm{CH}-\mathrm{C}_{6} \mathrm{H}_{3} \mathrm{~F}_{2}$ & $-\mathrm{CH}_{2} \mathrm{CH}_{3}$ & $0.97 \pm 0.06$ & $>200$ \\
\hline $7 \mathrm{~g}$ & $-\mathrm{SCH}_{2} \mathrm{CH}_{2} \mathrm{CH}_{3}$ & $-\mathrm{CH}\left(\mathrm{CH}_{2}\right) \mathrm{CH}-\mathrm{C}_{6} \mathrm{H}_{3} \mathrm{~F}_{2}$ & $-\mathrm{C}_{5} \mathrm{H}_{9}$ & $1.00 \pm 0.09$ & $>200$ \\
\hline $7 \mathrm{~h}$ & $-\mathrm{SCH}_{2} \mathrm{CH}_{2} \mathrm{CH}_{3}$ & $\mathrm{H}$ & $-\mathrm{C}_{5} \mathrm{H}_{6}(\mathrm{OH})_{3}$ & $1.10 \pm 0.09$ & $>200$ \\
\hline $7 \mathbf{i}$ & $-\mathrm{SCH}_{2} \mathrm{CH}_{2} \mathrm{CH}_{3}$ & $-\mathrm{CH}\left(\mathrm{CH}_{2}\right) \mathrm{CH}-\mathrm{C}_{6} \mathrm{H}_{3} \mathrm{~F}_{2}$ & $-\mathrm{C}_{5} \mathrm{H}_{6}(\mathrm{OH})_{3}$ & $4.80 \pm 2.50 \#$ & 25 \\
\hline $7 \mathbf{j}$ & $-\mathrm{SCH}_{2} \mathrm{CH}_{2} \mathrm{CH}_{3}$ & $\mathrm{H}$ & $-\mathrm{C}_{5} \mathrm{H}_{6}(\mathrm{OH})_{2} \mathrm{OCH}_{2} \mathrm{CH}_{2} \mathrm{OH}$ & $1.10 \pm 0.13$ & $>200$ \\
\hline $7 \mathbf{k}$ & $-\mathrm{SCH}_{2} \mathrm{CH}_{2} \mathrm{CH}_{3}$ & $-\mathrm{CH}\left(\mathrm{CH}_{2}\right)_{2}$ & $-\mathrm{C}_{5} \mathrm{H}_{6}(\mathrm{OH})_{2} \mathrm{OCH}_{2} \mathrm{CH}_{2} \mathrm{OH}$ & $2.60 \pm 0.65$ & $>200$ \\
\hline 71 & $-\mathrm{SCH}_{2} \mathrm{CH}_{2} \mathrm{CH}_{3}$ & $-\mathrm{CH}\left(\mathrm{CH}_{2}\right) \mathrm{CH}-\mathrm{C}_{6} \mathrm{H}_{5}$ & $-\mathrm{C}_{5} \mathrm{H}_{6}(\mathrm{OH})_{2} \mathrm{OCH}_{2} \mathrm{CH}_{2} \mathrm{OH}$ & $3.10 \pm 0.65 \#$ & $>200$ \\
\hline $7 \mathrm{~m}$ & $-\mathrm{SCH}_{2} \mathrm{CH}_{2} \mathrm{CH}_{3}$ & $-\mathrm{CH}_{2} \mathrm{CH}_{2}-\mathrm{C}_{6} \mathrm{H}_{3} \mathrm{~F}_{2}$ & $-\mathrm{C}_{5} \mathrm{H}_{6}(\mathrm{OH})_{2} \mathrm{OCH}_{2} \mathrm{CH}_{2} \mathrm{OH}$ & $1.10 \pm 0.07$ & $>200$ \\
\hline 1 & $-\mathrm{SCH}_{2} \mathrm{CH}_{2} \mathrm{CH}_{3}$ & $-\mathrm{CH}\left(\mathrm{CH}_{2}\right) \mathrm{CH}-\mathrm{C}_{6} \mathrm{H}_{3} \mathrm{~F}_{2}$ & $-\mathrm{C}_{5} \mathrm{H}_{6}(\mathrm{OH})_{2} \mathrm{OCH}_{2} \mathrm{CH}_{2} \mathrm{OH}$ & $4.30 \pm 2.20 \#$ & 25 \\
\hline $7 n$ & $-\mathrm{SCH}_{2} \mathrm{CH}(\mathrm{OH}) \mathrm{CH}_{3}$ & $\mathrm{H}$ & $-\mathrm{C}_{5} \mathrm{H}_{6}(\mathrm{OH})_{3}$ & $1.00 \pm 0.17$ & $>200$ \\
\hline 70 & $-\mathrm{SCH}_{2} \mathrm{CH}_{2} \mathrm{CH}_{2} \mathrm{OH}$ & $\mathrm{H}$ & $-\mathrm{C}_{5} \mathrm{H}_{6}(\mathrm{OH})_{3}$ & $1.00 \pm 0.09$ & $>200$ \\
\hline $7 p$ & $-\mathrm{SCH}_{2} \mathrm{CH}_{2} \mathrm{CH}_{2} \mathrm{OH}$ & $-\mathrm{CH}\left(\mathrm{CH}_{2}\right) \mathrm{CH}-\mathrm{C}_{6} \mathrm{H}_{3} \mathrm{~F}_{2}$ & $-\mathrm{C}_{5} \mathrm{H}_{6}(\mathrm{OH})_{3}$ & $2.70 \pm 0.99 * \#$ & $>200$ \\
\hline $7 q$ & $R-\mathrm{SCH}_{2} \mathrm{CH}(\mathrm{OH}) \mathrm{CH}_{3}$ & $-\mathrm{CH}\left(\mathrm{CH}_{2}\right) \mathrm{CH}-\mathrm{C}_{6} \mathrm{H}_{3} \mathrm{~F}_{2}$ & $-\mathrm{C}_{5} \mathrm{H}_{6}(\mathrm{OH})_{3}$ & $6.20 \pm 3.70 \#$ & $>200$ \\
\hline $7 \mathbf{r}$ & $\mathrm{S}-\mathrm{SCH}_{2} \mathrm{CH}(\mathrm{OH}) \mathrm{CH}_{3}$ & $-\mathrm{CH}\left(\mathrm{CH}_{2}\right) \mathrm{CH}-\mathrm{C}_{6} \mathrm{H}_{3} \mathrm{~F}_{2}$ & $-\mathrm{C}_{5} \mathrm{H}_{6}(\mathrm{OH})_{3}$ & $2.00 \pm 0.72 \#$ & $>200$ \\
\hline $7 \mathrm{~s}$ & $-\mathrm{SCH}_{2} \mathrm{CH}(\mathrm{OH}) \mathrm{CH}_{3}$ & $-\mathrm{CH}\left(\mathrm{CH}_{2}\right) \mathrm{CH}-\mathrm{C}_{6} \mathrm{H}_{3} \mathrm{~F}_{2}$ & $-\mathrm{C}_{5} \mathrm{H}_{6}(\mathrm{OH})_{2} \mathrm{OCH}_{2} \mathrm{CH}_{2} \mathrm{OH}$ & $1.70 \pm 0.24$ & $>200$ \\
\hline $7 t$ & $-\mathrm{SCH}_{2} \mathrm{CH}_{2} \mathrm{CH}_{2} \mathrm{OH}$ & $-\mathrm{CH}\left(\mathrm{CH}_{2}\right) \mathrm{CH}-\mathrm{C}_{6} \mathrm{H}_{3} \mathrm{~F}_{2}$ & $-\mathrm{C}_{5} \mathrm{H}_{6}(\mathrm{OH})_{2} \mathrm{OCH}_{2} \mathrm{CH}_{2} \mathrm{OH}$ & $1.00 \pm 0.02$ & $>200$ \\
\hline $7 u$ & $-\mathrm{H}$ & $-\mathrm{CH}\left(\mathrm{CH}_{2}\right) \mathrm{CH}-\mathrm{C}_{6} \mathrm{H}_{3} \mathrm{~F}_{2}$ & $-\mathrm{C}_{5} \mathrm{H}_{6}(\mathrm{OH})_{3}$ & $1.00 \pm 0.04$ & $>200$ \\
\hline $7 v$ & $-\mathrm{Cl}$ & $-\mathrm{CH}\left(\mathrm{CH}_{2}\right) \mathrm{CH}-\mathrm{C}_{6} \mathrm{H}_{3} \mathrm{~F}_{2}$ & $-\mathrm{C}_{5} \mathrm{H}_{6}(\mathrm{OH})_{3}$ & $1.10 \pm 0.12$ & $>200$ \\
\hline
\end{tabular}

\footnotetext{
a Vehicle: $1 \%$ DMSO.
}

b MIC: minimal inhibitory concentration. Antiplatelet activity is presented as fold-inhibition vs. vehicle of area under the curve values of ADP-induced platelet aggregation (mean \pm S.D., $\mathrm{n}=4-21$ ). \# indicates $P<0.05$ vs. vehicle; * indicates $P<0.05$ vs. antiplatelet activity of compound 1 (wilcoxon matched-pairs signed rank test). 


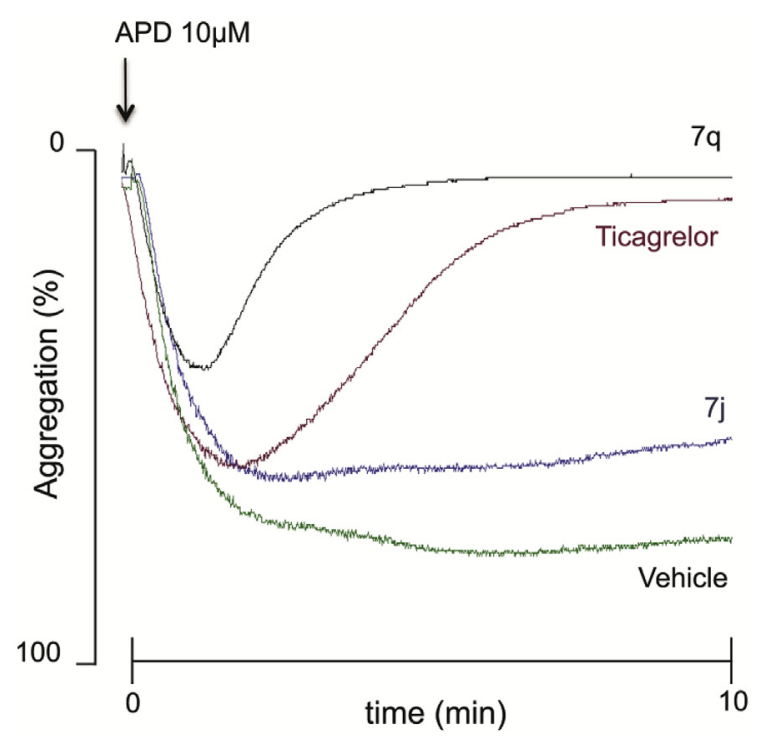

Fig. 4. Representative ADP-induced platelet aggregation curves performed in human citrated PRP after 10-min pre-incubation with the indicated molecules $(1.8 \mu \mathrm{M})$ or vehicle ( $1 \%$ DMSO). The arrow depicts the time of agonist addition.

of $200 \mu \mathrm{M}$. On the contrary, five ticagrelor analogues (7i, 71, 7p, 7q, 7r), among which the active metabolite AR-C124910 (7i), displayed antiplatelet activity. The antiplatelet activity of molecules $\mathbf{7 i}, \mathbf{7 1}, \mathbf{7 q}$ and $7 \mathbf{r}$ was similar to that of the reference compound $\mathbf{1}$ while it was weaker than the reference compound for molecule $7 \mathbf{p}(P=0.016)$.

Interestingly, the antiplatelet activity of the R-isomer $\mathbf{7 q}$ was found to be more pronounced than that of its counterpart, the Sisomer $7 \mathbf{r}(P=0.03)$, both of them being possible metabolites of ticagrelor and resulting from the hydroxylation at the 2-position of the propylsulfanyl side chain of the active metabolite 7i [22]. Hydroxylation at the 3-position of the same side chain of $\mathbf{7 i}$ also provided another known metabolite [22], compound 7p, which also showed antiplatelet activity.

The antiplatelet activity, but not the antibacterial activity, was maintained when the nature of the aralkylamino side chain at the 7-position was simplified. The ticagrelor analogue $\mathbf{7 1}$ devoid of the two fluorine atoms at the 3,4-positions of the phenyl ring was equipotent to the reference compound $\mathbf{1}$. The presence of a single cyclopropyl chain instead of a phenylcyclopropyl moiety (compound 7k) was sufficient to maintain a marked antiplatelet activity. Surprisingly, the replacement of the cyclopropyl group of $\mathbf{1}$ with an ethylene moiety (compound $\mathbf{7 m}$ ) dramatically suppressed the antiplatelet activity. The absence of the aralkyl group on the nitrogen atom at the 7-position of $\mathbf{1}$ (providing compound $\mathbf{7 j}$ ) also generated the similar results.

\section{Conclusion}

A series of 1,2,3-triazolo[4,5-d]pyrimidines structurally related to ticagrelor were synthesized and examined as putative antiplatelet and antibacterial agents. Slight modifications of the structure of ticagrelor dramatically led to a loss of the antibacterial activity against methicillin-resistant Staphylococcus aureus, while the antiplatelet activity was maintained with some simplified ticagrelor analogues. Our results indicated that the antiplatelet and antibacterial activity of 1,2,3-triazolo[4,5-d]pyrimidines were not necessarily linked supporting the view that the observed effects involve distinct mechanisms and unrelated biological targets.

\section{Experimental section}

\subsection{General procedures}

Melting points were determined on a Stuart SMP3 capillary apparatus and are uncorrected. The ${ }^{1} \mathrm{H}$ and ${ }^{13} \mathrm{C}$ NMR spectra were recorded on Bruker instruments equipped with a TCI cryoprobe (Bruker Avance HD $500 \mathrm{MHz}$ for ${ }^{1} \mathrm{H} ; 125 \mathrm{MHz}$ for ${ }^{13} \mathrm{C}$ /Bruker Avance III HD $700 \mathrm{MHz}$ for ${ }^{1} \mathrm{H}$; $176 \mathrm{MHz}$ for ${ }^{13} \mathrm{C}$ ) using deuterated dimethyl sulfoxide (DMSO- $\left.d_{6}\right)$ or deuterated chloroform $\left(\mathrm{CDCl}_{3}\right)$ as the solvent with tetramethylsilane (TMS) as an internal standard; chemical shifts are reported in $\delta$ values (ppm) relative to that of internal TMS. The abbreviations $\mathrm{s}=$ singlet, $\mathrm{d}=$ doublet, $\mathrm{t}=$ triplet, $\mathrm{q}=$ quadruplet, $\mathrm{p}=$ pentuplet, $\mathrm{h}=$ hexuplet, $\mathrm{m}=$ multiplet, $\mathrm{dd}=$ doublet of doublet, $\mathrm{td}=$ triplet of doublet, $\mathrm{qd}=$ quadruplet of doublet, $\mathrm{dt}=$ doublet of triplet, $\mathrm{dq}=$ doublet of quadruplet, $\mathrm{tt}=$ triplet of triplet, $\mathrm{tq}=$ triplet of quadruplet, $\mathrm{ddd}=$ doublet of doublet of doublets, ddt $=$ doublet of doublet of triplets and $\mathrm{bs}=$ broad singlet are used throughout. The compounds bearing an aralkylamino group at the 7-position shows existence of two isomeric forms, resulting in the duplication of several proton signals described as major and minor in the NMR data. Elemental analyses (C, H, N, S) were realized on a Thermo Scientific Flash EA 1112 elemental analyzer and were within $\pm 0.4 \%$ of the theoretical values for carbon, hydrogen and nitrogen; a higher tolerance $( \pm 0.75 \%)$ was admitted for sulfur, considering its corresponding peak shape. This analytical method certified a purity of $\geq 95 \%$ for each tested compound. All reactions were routinely checked by TLC on silica gel Merck 60 F254.

\subsection{Materials}

2-Thiobarbituric acid 8, 4,6-dichloro-2-(propylthio)pyrimidin5-amine $\mathbf{1 2 b} .3$ ( $\mathrm{R}^{1}=$ propyl), 4,6-dichloropyrimidin-5-amine 12c, 2,4,6-trichloro-5-nitropyrimidine 11a, $(1 R, 2 S)-2-(3,4-$ difluorophenyl)cyclopropanamine and 2-(( $3 \mathrm{a} R, 4 S, 6 R, 6 \mathrm{a} S)-6$-amino-2,2dimethyltetrahydro- $\quad 3 \mathrm{aH}$-cyclopenta[d][1,3]dioxol-4-yl)oxy) ethanol were purchased from Fluorochem. (3aR,4S,6R,6aS)-6Amino-2,2-dimethyltetrahydrocyclopenta[d][1,3]dioxol-4-ol was purchased from Spirochem. 3-mercapto-1-propanol and 1mercaptopropan-2-ol were purchased from Aldrich. Both enantiomers of 1-mercaptopropan-2-ol were purchased from Chemspace.

\subsection{2-(Methylthio)pyrimidine-4,6-diol (9.1)}

2-Thiobarbituric acid $\mathbf{8}$ (2,50 g, $17.4 \mathrm{mmol})$ was dissolved in $\mathrm{KOH}$ $10 \%(25 \mathrm{~mL})$ and supplemented with methyl iodide $(1.25 \mathrm{~mL}$, $20.0 \mathrm{mmol}$ ). The reaction mixture was introduced in a sealed vessel and heated at $80^{\circ} \mathrm{C}$ for $1 \mathrm{~h}$. After cooling on an ice bath to $5^{\circ} \mathrm{C}$, the mixture was acidified by addition of hydrochloric acid $6 \mathrm{~N}$ and the resulting precipitate was filtered off and washed with diethyl ether to give 9.1 (2,10 g, 77\% yield, m.p.: $\left.>300{ }^{\circ} \mathrm{C}\right) .{ }^{1} \mathrm{H}$ NMR (DMSO- $\left.d_{6}\right)$ : $\delta 2.46(\mathrm{~s}, 3 \mathrm{H}, \mathrm{SCH} 3), 5.13(\mathrm{~s}, 1 \mathrm{H}, \mathrm{CH}), 11.71(\mathrm{bs}, 2 \mathrm{H}, \mathrm{OH}) .{ }^{13} \mathrm{C}$ NMR (DMSO- $\left.d_{6}\right): \delta$ 12.7, 85.5, 158.6, 163.5.

\subsection{2-(Ethylthio)pyrimidine-4,6-diol (9.2)}

2-Thiobarbituric acid 8 (2.50 g, $17.4 \mathrm{mmol})$ was dissolved in $\mathrm{KOH}$ $10 \%(25 \mathrm{~mL})$ and supplemented with ethyl iodide $(1.63 \mathrm{~mL}$, $20.0 \mathrm{mmol}$ ). The reaction mixture was introduced in a sealed vessel and heated at $80^{\circ} \mathrm{C}$ for $1 \mathrm{~h}$. After cooling on an ice bath to $5^{\circ} \mathrm{C}$, the mixture was acidified by addition of hydrochloric acid $6 \mathrm{~N}$ and the resulting precipitate was filtered off and washed with diethyl ether to give 9.2 (2.05 g, 69\% yield, m.p.: $>300{ }^{\circ} \mathrm{C}$ ). ${ }^{1} \mathrm{H}$ NMR (DMSO-d 6 ): 
$\delta 1.28\left(\mathrm{t}, J=7.3 \mathrm{~Hz}, 3 \mathrm{H}, \mathrm{CH}_{3}\right), 3.08(\mathrm{q}, J=7.3 \mathrm{~Hz}, 2 \mathrm{H}, \mathrm{SCH} 2), 5.12(\mathrm{~s}$, $1 \mathrm{H}, \mathrm{CH}), 11.68$ (bs, $2 \mathrm{H}, \mathrm{OH}) .{ }^{13} \mathrm{C}$ NMR (DMSO- $\left.d_{6}\right): \delta 14.6,24.0,85.6$, $158.1,162.8$.

\subsection{2-(Methylthio)-5-nitropyrimidine-4,6-diol (10.1)}

To $6 \mathrm{~mL}$ of acetic acid cooled at $5^{\circ} \mathrm{C}$ on an ice bath were added fuming nitric acid (2.5 mL) and (9.1) $(2.0 \mathrm{~g}, 12.6 \mathrm{mmol})$. After $1 \mathrm{~h}$ stirring at room temperature, the mixture was cooled at $5{ }^{\circ} \mathrm{C}$ on an ice bath, water $(50 \mathrm{~mL})$ was added and the resulting precipitate was filtered off to give $\mathbf{1 0 . 1}\left(1,72 \mathrm{~g}, 67 \%\right.$ yield, m.p.: $220-221^{\circ} \mathrm{C}$ (dec.)). ${ }^{1} \mathrm{H}$ NMR $\left(\right.$ DMSO- $\left._{6}\right) \delta 2.56$ (s, 3H, SCH 3). ${ }^{13} \mathrm{C}$ NMR (DMSO-d $\left.d_{6}\right) \delta 13.2$, 117.4, 158.7, 164.6.

\subsection{2-(Ethylthio)-5-nitropyrimidine-4,6-diol (10.2)}

To $6 \mathrm{~mL}$ of acetic acid cooled at $5{ }^{\circ} \mathrm{C}$ on an ice bath were added fuming nitric acid $(2.5 \mathrm{~mL})$ and $(\mathbf{9 . 2})(1.80 \mathrm{~g}, 10.5 \mathrm{mmol})$. After $1 \mathrm{~h}$ stirring at room temperature, the mixture was cooled at $5{ }^{\circ} \mathrm{C}$ on an ice bath, water $(50 \mathrm{~mL})$ was added and the resulting precipitate was filtered off to give $\mathbf{1 0 . 2}\left(1.57 \mathrm{~g}, 69 \%\right.$ yield, m.p.: $210-213{ }^{\circ} \mathrm{C}$ (dec.)). ${ }^{1} \mathrm{H}$ NMR (DMSO- $\left.d_{6}\right): \delta 1.31\left(\mathrm{t}, J=7.3 \mathrm{~Hz}, 3 \mathrm{H}, \mathrm{CH}_{3}\right), 3.17(\mathrm{q}, J=7.3 \mathrm{~Hz}$, $2 \mathrm{H}, \mathrm{SCH} 2) .{ }^{13} \mathrm{C}$ NMR (DMSO-d $)$ ): $\delta 14.4,24.7,117.4,158.9,164.0$.

\subsection{4,6-Dichloro-2-(methylthio)-5-nitropyrimidine (11b.1)}

To a solution of (10.1) $(1.5 \mathrm{~g}, 7.4 \mathrm{mmol})$ in $\mathrm{POCl}_{3}(10 \mathrm{~mL})$ cooled at $5{ }^{\circ} \mathrm{C}$ on an ice bath was added dropwise 2,6-lutidine $(2.5 \mathrm{~mL})$. After $2 \mathrm{~h}$ stirring at $80^{\circ} \mathrm{C}$, the mixture was poured on crushed ice and the resulting precipitate was filtered off to give $\mathbf{1 1 b . 1}$ ( $1.63 \mathrm{~g}, 92 \%$ yield, m.p.: $\left.63-64{ }^{\circ} \mathrm{C}\right) .{ }^{1} \mathrm{H}$ NMR (DMSO- $\left.d_{6}\right): \delta 2.56$ (s, 3H, SCH 3). ${ }^{13} \mathrm{C}$ NMR (DMSO- $\left.d_{6}\right): \delta 13.5,149.0,154.5,166.1$.

\subsection{4,6-Dichloro-2-(ethylthio)-5-nitropyrimidine (11b.2)}

To a solution of $(\mathbf{1 0 . 2})(1.5 \mathrm{~g}, 6.9 \mathrm{mmol})$ in $\mathrm{POCl}_{3}(10 \mathrm{~mL})$ cooled at $5{ }^{\circ} \mathrm{C}$ on an ice bath was added dropwise 2,6-lutidine $(2.5 \mathrm{~mL})$. After $2 \mathrm{~h}$ stirring at $80^{\circ} \mathrm{C}$, the mixture was poured on crushed ice and extracted with ethyl acetate $(3 \times 50 \mathrm{~mL})$. The organic layers were washed with water and with an aqueous saturated solution of sodium hydrogenocarbonate and ethyl acetate was evaporated to dryness under vacuum. The resulting oily residue $\mathbf{1 1 b . 2}(1.76 \mathrm{~g}, 85 \%$ yield) was used without further purification in the next step (12b.2). ${ }^{1} \mathrm{H}$ NMR (DMSO-d $\left.d_{6}\right) \delta 1.35\left(\mathrm{t}, J=7.3 \mathrm{~Hz}, 3 \mathrm{H}, \mathrm{CH}_{3}\right), 3.18$ (q, $J=7.3 \mathrm{~Hz}, 2 \mathrm{H}, \mathrm{SCH} 2) .{ }^{13} \mathrm{C}$ NMR (DMSO-d $\left.d_{6}\right) \delta 14.0,25.3,149.1,154.5$, 165.4 .

\subsection{2,4,6-Trichloropyrimidin-5-amine (12a)}

To a solution of 2,4,6-trichloro-5-nitropyrimidine 11a (1.0 g, $4.38 \mathrm{mmol})$ in methanol $(10 \mathrm{~mL})$ and acetic acid $(4 \mathrm{~mL})$ was added iron powder $(1.0 \mathrm{~g}, 17.9 \mathrm{mmol})$ under vigorous agitation. After $1 \mathrm{~h}$ at room temperature, the mixture was diluted with methanol $(30 \mathrm{~mL})$ and filtered. The precipitate was washed with methanol $(30 \mathrm{~mL})$ and the filtrate was then concentrated under reduced pressure. The residue was taken up with water $(50 \mathrm{~mL})$ and extracted with dichloromethane $(3 \times 50 \mathrm{~mL})$. The combined organic layers were dried over $\mathrm{MgSO}_{4}$, evaporated and purified by column chromatography on silica gel using hexane/ethyl acetate gradient to give 12a as a white solid $\left(0.77 \mathrm{~g}, 89 \%\right.$ yield, m.p.: $\left.107-109{ }^{\circ} \mathrm{C}\right) .{ }^{1} \mathrm{H}$ NMR $\left(\mathrm{CDCl}_{3}\right): \delta 4.51$ (bs, $\left.2 \mathrm{H}, \mathrm{NH}_{2}\right) .{ }^{13} \mathrm{C} \mathrm{NMR}\left(\mathrm{CDCl}_{3}\right): \delta 134.7,145.1,145.4$.

\subsection{4,6-Dichloro-2-(methylthio)pyrimidin-5-amine (12b.1)}

To a solution of (11b.1) (1.0 g, $4.2 \mathrm{mmol})$ in methanol (10 mL) and acetic acid ( $4 \mathrm{~mL}$ ) was added iron powder (1,07 g, $19.5 \mathrm{mmol})$. After $1 \mathrm{~h}$ stirring at room temperature, ethyl acetate $(50 \mathrm{~mL})$ was added and the suspension was filtered. The filtrate was washed with water and with an aqueous saturated solution of sodium hydrogenocarbonate and the organic layer was evaporated to dryness under vacuum. Water was added on the residue and the resulting precipitate was filtered off to give $\mathbf{1 2 b . 1}(0.83 \mathrm{~g}, 95 \%$ yield, m.p.: $105-108^{\circ} \mathrm{C}$ ). ${ }^{1} \mathrm{H}$ NMR (DMSO- $\left.d_{6}\right): \delta 2.45$ (s, 3H, SCH 3), 5.90 (s, $2 \mathrm{H}, \mathrm{NH}_{2}$ ). ${ }^{13} \mathrm{C}$ NMR (DMSO-d $\left.d_{6}\right): \delta 13.8,133.4,143.7,154.4$.

\subsection{4,6-Dichloro-2-(ethylthio)pyrimidin-5-amine (12b.2.)}

To a solution of (11b.2) $(1.0 \mathrm{~g}, 3.9 \mathrm{mmol})$ in methanol $(10 \mathrm{~mL})$ and acetic acid $(4 \mathrm{~mL})$ was added iron powder $(1.07 \mathrm{~g}, 19.5 \mathrm{mmol})$. After $1 \mathrm{~h}$ stirring at room temperature, ethyl acetate $(50 \mathrm{~mL})$ was added and the suspension was filtered. The filtrate was washed with water and with an aqueous saturated solution of sodium hydrogenocarbonate and the organic layer was evaporated to dryness under vacuum. Water was added on the residue and the resulting precipitate was filtered off to give $\mathbf{1 2 b . 2}(0.76 \mathrm{~g}, 86 \%$ yield, m.p.: $\left.48-50{ }^{\circ} \mathrm{C}\right) .{ }^{1} \mathrm{H}$ NMR (DMSO- $\left.d_{6}\right): \delta 1.28\left(\mathrm{t}, J=7.3 \mathrm{~Hz}, 3 \mathrm{H}, \mathrm{CH}_{3}\right)$, $3.02(\mathrm{q}, J=7.3 \mathrm{~Hz}, 2 \mathrm{H}, \mathrm{SCH} 2), 5.90\left(\mathrm{~s}, 2 \mathrm{H}, \mathrm{NH}_{2}\right) .{ }^{13} \mathrm{C} \mathrm{NMR}$ (DMSO- $\left.d_{6}\right): \delta 14.3,24.9,133.5,143.7,153.8$.

4.12. (3aR,4S,6R,6aS)-6-[(5-amino-6-chloropyrimidin-4-yl)amino]2,2-dimethyltetrahydro-3aH-cyclopenta[d][1,3]dioxol-4-ol (13c.1)

The mixture of 4,6-dichloropyrimidin-5-amine $(1.0 \mathrm{~g}$, $6.1 \mathrm{mmol}), \quad(3 \mathrm{a} R, 4 S, 6 R, 6 \mathrm{a} S)-6$-amino-2,2-dimethyltetrahydro cyclopenta[d][1,3]dioxol-4-ol (1.34 g, $7.8 \mathrm{mmol})$ and triethylamine $(0.85 \mathrm{~mL}, 6.1 \mathrm{mmol})$ in acetonitrile $(10 \mathrm{~mL})$ was placed in a sealed vessel and heated overnight at $110{ }^{\circ} \mathrm{C}$. After evaporation of the solvent, the residue was purified by column chromatography on silica gel using $100 \%$ ethyl acetate to give $\mathbf{1 3 c . 1}$ as a white solid (1.43 g, 78\% yield). ${ }^{1} \mathrm{H}$ NMR (DMSO- $\left.d_{6}\right): \delta 1.21\left(\mathrm{~s}, 3 \mathrm{H}, \mathrm{CH}_{3}\right), 1.36$ (s, $\left.3 \mathrm{H}, \mathrm{CH}_{3}\right), 1.70\left(\mathrm{~d}, J=13.7 \mathrm{~Hz}, 1 \mathrm{H}, 5^{\prime}-\mathrm{Ha}\right), 2.22\left(\mathrm{~m}, 1 \mathrm{H}, 5^{\prime}-\mathrm{Hb}\right), 4.07(\mathrm{~m}$, $\left.1 \mathrm{H}, 4^{\prime}-H\right), 4.29\left(\mathrm{t}, J=5.9 \mathrm{~Hz}, 1 \mathrm{H}, 6^{\prime}-H\right), 4.41\left(\mathrm{~d}, J=6.0 \mathrm{~Hz}, 1 \mathrm{H}, 3 \mathrm{a}^{\prime}-H\right)$, $4.51\left(\mathrm{~d}, J=6.0 \mathrm{~Hz}, 1 \mathrm{H}, 6 \mathrm{a}^{\prime}-H\right), 4.97\left(\mathrm{~s}, 2 \mathrm{H}, \mathrm{NH}_{2}\right), 5.25(\mathrm{~d}, J=3.1 \mathrm{~Hz}$, $1 \mathrm{H}, \mathrm{OH}), 6.49(\mathrm{~d}, J=7.2 \mathrm{~Hz}, 1 \mathrm{H}, \mathrm{NH}), 7.79(\mathrm{~s}, 1 \mathrm{H}, 2-\mathrm{H}) .{ }^{13} \mathrm{C}$ NMR (DMSO- $\left.d_{6}\right)$ : $\delta$ 24.1, 26.5, 36.0, 57.0, 75.3, 84.4, 85.7, 109.7, 123.5, 137.6, 146.1, 151.7 .

4.13. (3aR,4S,6R,6aS)-6-((5-amino-2,6-dichloropyrimidin-4-yl) amino)-2,2-dimethyltetrahydro-3aH-cyclopenta[d][1,3]dioxol-4-ol (13a.1)

The mixture of 2,4,6-trichloropyrimidin-5-amine 12a (1.0 g, $5.0 \quad \mathrm{mmol}), \quad(3 \mathrm{a} R, 4 S, 6 R, 6 \mathrm{aS})-6-\quad$ amino-2,2dimethyltetrahydrocyclopenta[d][1,3]dioxol-4-ol (1.1 g, $6.4 \mathrm{mmol})$ and triethylamine $(0.7 \mathrm{~mL}, 5.0 \mathrm{mmol})$ in acetonitrile $(10 \mathrm{~mL})$ was placed in a sealed vessel and heated overnight at $110{ }^{\circ} \mathrm{C}$. After evaporation of the solvent, the residue was purified by column chromatography on silica gel using $100 \%$ ethyl acetate to give $13 a .1$ as a white solid (1.48 g, 88\% yield, m.p.: $105{ }^{\circ} \mathrm{C}$ (dec.)). ${ }^{1} \mathrm{H}$ NMR $\left(\mathrm{CDCl}_{3}\right): \delta 1.29\left(\mathrm{~s}, 3 \mathrm{H}, \mathrm{CH}_{3}\right), 1.45\left(\mathrm{~s}, 3 \mathrm{H}, \mathrm{CH}_{3}\right), 1.82(\mathrm{~d}, J=14.5 \mathrm{~Hz}, 1 \mathrm{H}$, $\left.5^{\prime}-\mathrm{Ha}\right), 2.17(\mathrm{~d}, J=2.0 \mathrm{~Hz}, 1 \mathrm{H}, \mathrm{OH}), 2.36(\mathrm{dt}, J=14.5 \mathrm{~Hz} / 5.2 \mathrm{~Hz}, 1 \mathrm{H}$, $\left.5^{\prime}-\mathrm{Hb}\right), 3.28\left(\mathrm{~s}, 2 \mathrm{H}, \mathrm{NH}_{2}\right), 4.40\left(\mathrm{~d}, J=2.0 \mathrm{~Hz}, 1 \mathrm{H}, 4^{\prime}-\mathrm{H}\right), 4.54(\mathrm{~d}$, $\left.J=5.3 \mathrm{~Hz}, 1 \mathrm{H}, 3 \mathrm{a}^{\prime}-H\right), 4.57\left(\mathrm{~d}, J=5.3 \mathrm{~Hz}, 1 \mathrm{H}, 6 \mathrm{a}^{\prime}-H\right), 4.71(\mathrm{dd}$, $\left.J=8.4 \mathrm{~Hz} / 6.6 \mathrm{~Hz}, 1 \mathrm{H}, 6^{\prime}-\mathrm{H}\right), 6.11(\mathrm{~d}, J=8.7 \mathrm{~Hz}, 1 \mathrm{H}, \mathrm{NH}) .{ }^{13} \mathrm{C} \mathrm{NMR}$ $\left(\mathrm{CDCl}_{3}\right): \delta 23.8,26.2,35.0,57.0,78.1,85.2,86.1,110.6,120.3,142.8$, 150.2, 155.1 . 
4.14. 2-(((3aR,4S,6R,6aS)-6-((5-amino-2,6-dichloropyrimidin-4-yl) amino)-2,2-dimethyltetrahydro-3aH-cyclopenta[d][1,3]dioxol-4-yl) oxy)ethanol (13a.2)

The mixture of 2,4,6-trichloropyrimidin-5-amine 12a (1.0 g, $5.0 \mathrm{mmol}), 2-(((3 \mathrm{a} R, 4 S, 6 R, 6 \mathrm{a} S)-6$-amino-2,2-dimethyltetrahydro$3 \mathrm{aH}$-cyclopenta[d][1,3]dioxol-4-yl)oxy)ethanol (1.4 g, $6.4 \mathrm{mmol})$ and triethylamine $(0.7 \mathrm{~mL}, 5.0 \mathrm{mmol})$ in acetonitrile $(10 \mathrm{~mL})$ was placed in a sealed vessel and heated overnight at $110{ }^{\circ} \mathrm{C}$. After evaporation of the solvent, the residue was purified by column chromatography on silica gel using $100 \%$ ethyl acetate to give $13 a .2$ as an oily residue ( $1.59 \mathrm{~g}, 83 \%$ yield). ${ }^{1} \mathrm{H}$ NMR (DMSO- $\left.d_{6}\right): \delta 1.23(\mathrm{~s}$, $\left.3 \mathrm{H}, \mathrm{CH}_{3}\right), 1.39\left(\mathrm{~s}, 3 \mathrm{H}, \mathrm{CH}_{3}\right), 1.86\left(\mathrm{~d}, \mathrm{~J}=14.0 \mathrm{~Hz}, 1 \mathrm{H}, 5^{\prime}-\mathrm{Ha}\right), 2.22(\mathrm{~m}$, $\left.1 \mathrm{H}, 5^{\prime}-\mathrm{Hb}\right), 3.51\left(\mathrm{~m}, 4 \mathrm{H}, \mathrm{OCH}_{2} \mathrm{CH}_{2} \mathrm{OH}\right), 3.90\left(\mathrm{~s}, 1 \mathrm{H}, 4^{\prime}-\mathrm{H}\right), 4.30(\mathrm{~s}, 1 \mathrm{H}$, $\left.6^{\prime}-H\right), 4.50\left(\mathrm{~s}, 1 \mathrm{H}, 6 \mathrm{a}^{\prime}-\mathrm{H}\right), 4.54\left(\mathrm{~s}, 1 \mathrm{H}, 3 \mathrm{a}^{\prime}-\mathrm{H}\right), 5.04\left(\mathrm{~s}, 1 \mathrm{H}, \mathrm{OCH}_{2-}\right.$ $\left.\mathrm{CH}_{2} \mathrm{OH}\right), 5.17\left(\mathrm{~s}, 2 \mathrm{H}, \mathrm{NH}_{2}\right), 6.83(\mathrm{~d}, J=6.7 \mathrm{~Hz}, 1 \mathrm{H}, \mathrm{NH}) .{ }^{13} \mathrm{C}$ NMR (DMSO- $d_{6}$ ): $\delta 24.1,26.3,32.9,56.6,60.3,70.3,83.2,83.5,83.9,110.2$, $112.8,136.5,144.6,152.7$.

\subsection{6-Chloro- $N^{4}$-methyl-2-(methylthio)pyrimidine-4,5-diamine} (13b.1)

4,6-Dichloro-2-(methylthio)pyrimidin-5-amine (12b.1) (0.5 g, $2.4 \mathrm{mmol}$ ) was dissolved in methanol $(2 \mathrm{~mL})$ and supplemented with a solution of methylamine $33 \% \mathrm{w} / \mathrm{w}$ in methanol $(0.87 \mathrm{~mL}$, $7.2 \mathrm{mmol}$ ). The reaction mixture was introduced in a sealed vessel and heated at $100{ }^{\circ} \mathrm{C}$ for $1 \mathrm{~h}$. After concentration of the reaction mixture to dryness under vacuum, the residue was purified by silica gel column chromatography to give $\mathbf{1 3 b . 1}$ ( $0.4 \mathrm{~g}, 82 \%$ yield, m.p.: 141-143 ${ }^{\circ} \mathrm{C}$ ). ${ }^{1} \mathrm{H}$ NMR (DMSO-d $\left.d_{6}\right): \delta 2.38$ (s, 3H, SCH 3), 2.88 (d, $\left.J=3.0 \mathrm{~Hz}, 3 \mathrm{H}, \mathrm{NHCH}_{3}\right), 4.70\left(\mathrm{~s}, 2 \mathrm{H}, \mathrm{NH}_{2}\right), 7.01(\mathrm{~s}, 1 \mathrm{H}, \mathrm{NH}) .{ }^{13} \mathrm{C} \mathrm{NMR}$ (DMSO- $\left.d_{6}\right): \delta 13.5,27.8,120.1,137.2,153.3,155.9$.

\subsection{6-Chloro- $\mathrm{N}^{4}$-ethyl-2-(methylthio)pyrimidine-4,5-diamine} (13b.2)

4,6-Dichloro-2-(methylthio)pyrimidin-5-amine (12b.1) (0.5 g, $2.4 \mathrm{mmol}$ ) was dissolved in a solution of ethylamine $2.0 \mathrm{M}$ in methanol ( $3.6 \mathrm{~mL}, 7.2 \mathrm{mmol})$. The reaction mixture was introduced in a sealed vessel and heated at $100{ }^{\circ} \mathrm{C}$ for $1 \mathrm{~h}$. After concentration of the reaction mixture to dryness under vacuum, the residue was purified by silica gel column chromatography to give $\mathbf{1 3 b . 2}(0.45 \mathrm{~g}$, 86\% yield, m.p.: $120-122{ }^{\circ} \mathrm{C}$ ). ${ }^{1} \mathrm{H}$ NMR (DMSO- $\left.d_{6}\right): \delta 1.16(\mathrm{t}$, $\left.J=7.2 \mathrm{~Hz}, 3 \mathrm{H}, \mathrm{NHCH}_{2} \mathrm{CH}_{3}\right), 2.37$ (s, 3H, SCH 3), 3.38 (qd, $J=7.2 \mathrm{~Hz}$ ) $\left.5.3 \mathrm{~Hz}, 2 \mathrm{H}, \mathrm{NHCH}_{2} \mathrm{CH}_{3}\right), 4.76\left(\mathrm{~s}, 2 \mathrm{H}, \mathrm{NH}_{2}\right), 6.94(\mathrm{t}, J=4.8 \mathrm{~Hz}, 1 \mathrm{H}, \mathrm{NH})$. ${ }^{13} \mathrm{C}$ NMR (DMSO- $d_{6}$ ): $\delta$ 13.5, 14.3, 35.7, 119.8, 137.2, 152.4, 155.7.

\subsection{6-Chloro-2-(ethylthio)- $\mathrm{N}^{4}$-methylpyrimidine-4,5-diamine} (13b.3)

4,6-Dichloro-2-(ethylthio)pyrimidin-5-amine (12b.2) (0.5 g, $2.2 \mathrm{mmol})$ was dissolved in methanol $(2 \mathrm{~mL})$ and supplemented with a solution of methylamine $33 \% \mathrm{w} / \mathrm{w}$ in methanol $(0.80 \mathrm{~mL}$, $6.6 \mathrm{mmol}$ ). The reaction mixture was introduced in a sealed vessel and heated at $100{ }^{\circ} \mathrm{C}$ for $1 \mathrm{~h}$. After concentration of the reaction mixture to dryness under vacuum, the residue was purified by silica gel column chromatography to give $\mathbf{1 3 b . 3}(0.43 \mathrm{~g}, 87 \%$ yield, m.p.: $112-114{ }^{\circ} \mathrm{C}$ ). ${ }^{1} \mathrm{H}$ NMR (DMSO- $\left.d_{6}\right): \delta 1.27\left(\mathrm{t}, J=7.2 \mathrm{~Hz}, 3 \mathrm{H}, \mathrm{SCH}_{2} \mathrm{CH}_{3}\right)$, $2.87\left(\mathrm{~d}, J=3.8 \mathrm{~Hz}, 3 \mathrm{H}, \mathrm{NHCH}_{3}\right), 2.97$ (q, $J=7.2 \mathrm{~Hz}, 2 \mathrm{H}, \mathrm{SCH}_{2} \mathrm{CH}_{3}$ ), $4.70\left(\mathrm{~s}, 2 \mathrm{H}, \mathrm{NH}_{2}\right), 7.00(\mathrm{~s}, 1 \mathrm{H}, \mathrm{NH}) .{ }^{13} \mathrm{C}$ NMR (DMSO-d $\left.\mathrm{d}_{6}\right): \delta 14.9,24.5$, 27.8, 120.1, 137.2, 153.3, 155.4 .

\subsection{6-Chloro- $N^{4}$-ethyl-2-(ethylthio)pyrimidine-4,5-diamine (13b.4)}

4,6-Dichloro-2-(ethylthio)pyrimidin-5-amine (12b.2) (0.5 g, $2.2 \mathrm{mmol}$ ) was dissolved in a solution of ethylamine $2.0 \mathrm{M}$ in methanol (3.3 $\mathrm{mL}, 6.6 \mathrm{mmol})$. The reaction mixture was introduced in a sealed vessel and heated at $100{ }^{\circ} \mathrm{C}$ for $1 \mathrm{~h}$. After concentration of the reaction mixture to dryness under vacuum, the residue was purified by silica gel column chromatography to give $\mathbf{1 3 b . 4}(0.47 \mathrm{~g}$, 91\% yield, m.p.: $93-95^{\circ} \mathrm{C}$ ). ${ }^{1} \mathrm{H}$ NMR (DMSO- $\left.d_{6}\right): \delta 1.16(\mathrm{t}, J=7.2 \mathrm{~Hz}$, $\left.3 \mathrm{H}, \mathrm{NHCH}_{2} \mathrm{CH}_{3}\right), 1.27\left(\mathrm{t}, J=7.2 \mathrm{~Hz}, 3 \mathrm{H}, \mathrm{SCH}_{2} \mathrm{CH}_{3}\right), 2.96(\mathrm{q}, J=7.2 \mathrm{~Hz}$, $2 \mathrm{H}, \mathrm{SCH}_{2} \mathrm{CH}_{3}$ ), 3.38 (p, $\left.J=6.1 \mathrm{~Hz}, 2 \mathrm{H}, \mathrm{NHCH}_{2} \mathrm{CH}_{3}\right), 4.74\left(\mathrm{~s}, 2 \mathrm{H}, \mathrm{NH}_{2}\right.$ ), $6.93(\mathrm{~s}, 1 \mathrm{H}, \mathrm{NH}) .{ }^{13} \mathrm{C}$ NMR (DMSO- $\left.d_{6}\right): \delta 14.4,15.0,24.5,35.8,119.9$, 137.3, 152.6, 155.2.

\subsection{6-Chloro- $N^{4}$-methyl-2-(propylthio)pyrimidine-4,5-diamine (13b.5)}

4,6-Dichloro-2-(propylthio)pyrimidin-5-amine $\mathbf{1 2 b} .3$ (0.5 g, $2.1 \mathrm{mmol}$ ) was dissolved in methanol $(2 \mathrm{~mL})$ and supplemented with a solution of methylamine $33 \% \mathrm{w} / \mathrm{w}$ in methanol $(0.76 \mathrm{~mL}$, $6.3 \mathrm{mmol}$ ). The reaction mixture was introduced in a sealed vessel and heated at $100{ }^{\circ} \mathrm{C}$ for $1 \mathrm{~h}$. After concentration of the reaction mixture to dryness under vacuum, the residue was purified by silica gel column chromatography to give $\mathbf{1 3 b . 5}$ (0.47 g, 96\% yield, m.p.: 119-121 ${ }^{\circ} \mathrm{C}$ ). ${ }^{1} \mathrm{H}$ NMR (DMSO- $d_{6}$ ): $\delta 0.95(\mathrm{t}, J=7.4 \mathrm{~Hz}, 3 \mathrm{H}$, $\mathrm{SCH}_{2} \mathrm{CH}_{2} \mathrm{CH}_{3}$ ), 1.64 (h, $J=7.3 \mathrm{~Hz}, 2 \mathrm{H}, \mathrm{SCH}_{2} \mathrm{CH}_{2} \mathrm{CH}_{3}$ ), 2.87 (d, $\left.J=4.5 \mathrm{~Hz}, 3 \mathrm{H}, \mathrm{NHCH}_{3}\right), 2.96\left(\mathrm{t}, J=7.2 \mathrm{~Hz}, 2 \mathrm{H}, \mathrm{SCH}_{2} \mathrm{CH}_{2} \mathrm{CH}_{3}\right), 4.71(\mathrm{~s}$, $2 \mathrm{H}, \mathrm{NH}_{2}$ ), 7.01 (q, $\left.J=4.4 \mathrm{~Hz}, 1 \mathrm{H}, \mathrm{NHCH}_{3}\right) .{ }^{13} \mathrm{C}$ NMR (DMSO- $d_{6}$ ): $\delta$ 13.3, 22.6, 27.8, 32.1, 120.0, 137.1, 153.2, 155.4.

\subsection{6-Chloro- $\mathrm{N}^{4}$-ethyl-2-(propylthio)pyrimidine-4,5-diamine} (13b.6)

4,6-Dichloro-2-(propylthio)pyrimidin-5-amine 12b.3 (0.5 g, $2.1 \mathrm{mmol}$ ) was dissolved in a solution of ethylamine $2.0 \mathrm{M}$ in methanol (3.2 mL, $6.4 \mathrm{mmol}$ ). The reaction mixture was introduced in a sealed vessel and heated at $100{ }^{\circ} \mathrm{C}$ for $1 \mathrm{~h}$. After concentration of the reaction mixture to dryness under vacuum, the residue was purified by silica gel column chromatography to give $13 \mathbf{b . 6}(0.4 \mathrm{~g}$, 77\% yield, m.p.: $96-98{ }^{\circ} \mathrm{C}$ ). ${ }^{1} \mathrm{H}$ NMR (DMSO- $\left.d_{6}\right): \delta 0.95(\mathrm{t}, J=7.4 \mathrm{~Hz}$, $\left.3 \mathrm{H}, \mathrm{SCH}_{2} \mathrm{CH}_{2} \mathrm{CH}_{3}\right), 1.16$ (t, $\left.J=7.2 \mathrm{~Hz}, 3 \mathrm{H}, \mathrm{NHCH}_{2} \mathrm{CH}_{3}\right), 1.63$ (h, $\left.J=7.3 \mathrm{~Hz}, 2 \mathrm{H}, \mathrm{SCH}_{2} \mathrm{CH}_{2} \mathrm{CH}_{3}\right), 2.94\left(\mathrm{t}, J=7.2 \mathrm{~Hz}, 2 \mathrm{H}, \mathrm{SCH}_{2} \mathrm{CH}_{2} \mathrm{CH}_{3}\right)$, $3.37\left(\mathrm{~m}, 2 \mathrm{H}, \mathrm{NHCH}_{2} \mathrm{CH}_{3}\right), 4.75\left(\mathrm{~s}, 2 \mathrm{H}, \mathrm{NH}_{2}\right), 6.95(\mathrm{t}, J=4.8 \mathrm{~Hz}, 1 \mathrm{H}$, $\left.\mathrm{NHCH}_{2} \mathrm{CH}_{3}\right) .{ }^{13} \mathrm{C}$ NMR (DMSO-d $): \delta 13.3,14.3,22.7,32.1,35.7,119.8$, 137.3, 152.5, 155.3.

\subsection{6-Chloro- $N^{4}$-cyclopentyl-2-(propylthio)pyrimidine-4,5- diamine (13b.7)}

4,6-Dichloro-2-(propylthio)pyrimidin-5-amine $\mathbf{1 2 b} .3$ (0.5 g, $2.1 \mathrm{mmol})$ was dissolved in methanol $(2 \mathrm{~mL})$ and supplemented with cyclopentylamine $(536.0 \mathrm{mg}, 6.3 \mathrm{mmol})$. The reaction mixture was introduced in a sealed vessel and heated at $100^{\circ} \mathrm{C}$ for $2 \mathrm{~h}$. After concentration of the reaction mixture to dryness under vacuum, the residue was purified by silica gel column chromatography to give $13 \mathrm{~b} .7$ (0.57 g, 95\% yield, oil). ${ }^{1} \mathrm{H}$ NMR (DMSO- $\left.d_{6}\right): \delta 0.95(\mathrm{t}$, $\left.J=7.4 \mathrm{~Hz}, 3 \mathrm{H}, \mathrm{SCH}_{2} \mathrm{CH}_{2} \mathrm{CH}_{3}\right), 1.49\left(\mathrm{~m}, 2 \mathrm{H}, 2^{\prime}-\mathrm{Ha} / 5^{\prime}-\mathrm{Ha}\right), 1.55(\mathrm{~m}, 2 \mathrm{H}$, $\left.3^{\prime}-\mathrm{Ha} / 4^{\prime}-\mathrm{Ha}\right), 1.64\left(\mathrm{~m}, 2 \mathrm{H}, \mathrm{SCH}_{2} \mathrm{CH}_{2} \mathrm{CH}_{3}\right), 1.70\left(\mathrm{~m}, 2 \mathrm{H}, 3^{\prime}-\mathrm{Hb} / 4^{\prime}-\mathrm{Hb}\right)$, $1.96\left(\mathrm{~m}, 2 \mathrm{H}, 2^{\prime}-\mathrm{Hb} / 5^{\prime}-\mathrm{Hb}\right), 2.94\left(\mathrm{t}, J=7.2 \mathrm{~Hz}, 2 \mathrm{H}, \mathrm{SCH}_{2} \mathrm{CH}_{2} \mathrm{CH}_{3}\right), 4.25$ $\left(\mathrm{h}, J=6.7 \mathrm{~Hz}, 1 \mathrm{H}, 1^{\prime}-H\right), 4.83\left(\mathrm{~s}, 2 \mathrm{H}, \mathrm{NH}_{2}\right), 6.76(\mathrm{~d}, J=6.3 \mathrm{~Hz}, 1 \mathrm{H}, \mathrm{NH})$. ${ }^{13} \mathrm{C}$ NMR (DMSO- $\left.d_{6}\right): \delta 13.3,22.9,23.5,32.1,32.2,52.7,119.9,137.2$, 152.0, 155.0. 
4.22. (3aR,4S,6R,6aS)-6-((5-amino-6-chloro-2-(propylthio) pyrimidin-4-yl)amino)-2,2-dimethyltetrahydro-3aH-cyclopenta[d] [1,3]dioxol-4-ol (13b.8)

The mixture of 4,6-dichloro-2-(propylthio)pyrimidin-5-amine 12b.3 (1.0 g, $4.2 \mathrm{mmol}), \quad(3 \mathrm{a} R, 4 S, 6 R, 6 \mathrm{a} S)-6$-amino-2,2dimethyltetrahydrocyclopenta[ $d][1,3]$ dioxol-4-ol $\quad(0.93 \quad \mathrm{~g}$, $5.4 \mathrm{mmol})$ and triethylamine $(0.6 \mathrm{~mL}, 4.2 \mathrm{mmol})$ in acetonitrile $(10 \mathrm{~mL})$ was introduced in a sealed vessel and heated overnight at $110^{\circ} \mathrm{C}$. After evaporation of the solvent, the residue was purified by column chromatography on silica gel using $100 \%$ ethyl acetate to give 13b.8 as a brown oil (1.40 g, $89 \%$ yield, oil). ${ }^{1} \mathrm{H}$ NMR $\left(\mathrm{CDCl}_{3}\right)$ : $\delta 1.03\left(\mathrm{t}, J=7.4 \mathrm{~Hz}, 3 \mathrm{H}, \mathrm{SCH}_{2} \mathrm{CH}_{2} \mathrm{CH}_{3}\right), 1.26\left(\mathrm{~s}, 3 \mathrm{H}, \mathrm{CH}_{3}\right), 1.43(\mathrm{~s}, 3 \mathrm{H}$, $\left.\mathrm{CH}_{3}\right), 1.76\left(\mathrm{~m}, 2 \mathrm{H}, \mathrm{SCH}_{2} \mathrm{CH}_{2} \mathrm{CH}_{3}\right), 1.83\left(\mathrm{~d}, \mathrm{~J}=14.5 \mathrm{~Hz}, 1 \mathrm{H}, 5^{\prime}-\mathrm{Ha}\right), 2.14$ (s, $1 \mathrm{H}, \mathrm{OH}$ ), $2.36\left(\mathrm{~m}, 1 \mathrm{H}, 5^{\prime}-H b\right), 3.01$ (ddd, $J=13.4 \mathrm{~Hz} / 8.3 \mathrm{~Hz} / 6.4 \mathrm{~Hz}$, $1 \mathrm{H}, \mathrm{SCHa}$ ), 3.09 (s, $2 \mathrm{H}, \mathrm{NH}_{2}$ ), 3.13 (ddd, $J=13.5 \mathrm{~Hz} / 8.3 \mathrm{~Hz} / 6.4 \mathrm{~Hz}, 1 \mathrm{H}$, $\mathrm{SCHb}), 4.38$ (s, 1H, $\left.4^{\prime}-H\right), 4.51$ (dd, $\left.J=5.4 \mathrm{~Hz} / 1.7 \mathrm{~Hz}, 1 \mathrm{H}, 3 \mathrm{a}^{\prime}-H\right), 4.57$ (dd, $\left.J=5.4 \mathrm{~Hz} / 1.2 \mathrm{~Hz}, 1 \mathrm{H}, 6 \mathrm{a}^{\prime}-H\right), 4.62\left(\mathrm{t}, J=7.4 \mathrm{~Hz}, 1 \mathrm{H}, 6^{\prime}-H\right), 5.93$ $(\mathrm{d}, J=8.4 \mathrm{~Hz}, 1 \mathrm{H}, \mathrm{NH}) .{ }^{13} \mathrm{C} \mathrm{NMR}\left(\mathrm{CDCl}_{3}\right): \delta 13.5,23.2,23.8,26.2,33.2$, 35.0, 57.2, 78.1, 85.2, 86.2, 110.3, 117.2, 144.4, 154.5, 162.0.

\subsection{2-(((3aR,4S,6R,6aS)-6-((5-amino-6-chloro-2-(propylthio)} pyrimidin-4-yl)amino)-2,2-dimethyltetrahydro-3aH-cyclopenta[d] [1,3]dioxol-4-yl)oxy)ethanol (13b.9)

The mixture of 4,6-dichloro-2-(propylthio)pyrimidin-5-amine $12 \mathrm{~b} .3 \quad(1.0 \mathrm{~g}, \quad 4.2 \mathrm{mmol}), \quad 2-(((3 \mathrm{a} R, 4 S, 6 R, 6 \mathrm{a} S)-6$-amino-2,2dimethyltetrahydro-3aH-cyclopenta[ $d][1,3]$ dioxol-4-yl)oxy) ethanol ( $1.17 \mathrm{~g}, 5.4 \mathrm{mmol})$ and triethylamine $(0.6 \mathrm{~mL}, 4.2 \mathrm{mmol})$ in acetonitrile $(10 \mathrm{~mL})$ was introduced in a sealed vessel and heated overnight at $110{ }^{\circ} \mathrm{C}$. After evaporation of the solvent, the residue was purified by column chromatography on silica gel using $100 \%$ ethyl acetate to give 13b.9 (1.36 g, 77\% yield, m.p.: $\left.112-114{ }^{\circ} \mathrm{C}\right) .{ }^{1} \mathrm{H}$ $\operatorname{NMR}\left(\mathrm{CDCl}_{3}\right) \delta 1.03\left(\mathrm{t}, J=7.4 \mathrm{~Hz}, 3 \mathrm{H}, \mathrm{SCH}_{2} \mathrm{CH}_{2} \mathrm{CH}_{3}\right), 1.26\left(\mathrm{~s}, 3 \mathrm{H}, \mathrm{CH}_{3}\right)$, $1.43\left(\mathrm{~s}, 3 \mathrm{H}, \mathrm{CH}_{3}\right), 1.75\left(\mathrm{~m}, 2 \mathrm{H}, \mathrm{SCH}_{2} \mathrm{CH}_{2} \mathrm{CH}_{3}\right), 1.92(\mathrm{~d}, J=14.5 \mathrm{~Hz}, 1 \mathrm{H}$, $5^{\prime}-\mathrm{Ha}$ ), 2.28 (ddd, $J=14.5 \mathrm{~Hz} / 5.9 \mathrm{~Hz} / 4.4 \mathrm{~Hz}, 1 \mathrm{H}, 5^{\prime}-\mathrm{Hb}$ ), 2.59 (bs, $1 \mathrm{H}$, $\mathrm{OH}$ ), 2.99 (ddd, $J=13.4 \mathrm{~Hz} / 8.2 \mathrm{~Hz} / 6.4 \mathrm{~Hz}, 1 \mathrm{H}, \mathrm{SCHa}$ ), 3.14 (ddd, $J=13.5 \mathrm{~Hz} / 8.2 \mathrm{~Hz} / 6.4 \mathrm{~Hz}, 1 \mathrm{H}, \mathrm{SCHb}$ ), 3.38 (bs, $2 \mathrm{H}, \mathrm{NH} \mathrm{H}_{2}$ ), 3.60 (ddd, $J=9.9 \mathrm{~Hz} / 6.1 \mathrm{~Hz} / 2.6 \mathrm{~Hz}, 1 \mathrm{H}, \mathrm{OCHa}$ ), 3.70 (ddd, $J=9.9 \mathrm{~Hz} / 5.8 \mathrm{~Hz} /$ $2.5 \mathrm{~Hz}, 1 \mathrm{H}, \mathrm{OCHb}), 3.79\left(\mathrm{~m}, 2 \mathrm{H}, \mathrm{OCH}_{2} \mathrm{CH}_{2} \mathrm{OH}\right), 3.97(\mathrm{~d}, J=4.1 \mathrm{~Hz}, 1 \mathrm{H}$, $\left.4^{\prime}-H\right), 4.53$ (dd, $\left.J=5.4 \mathrm{~Hz} / 1.2 \mathrm{~Hz}, 1 \mathrm{H}, 3 \mathrm{a}^{\prime}-H\right), 4.59\left(\mathrm{~m}, 1 \mathrm{H}, 6^{\prime}-H\right), 4.61$ $\left(\mathrm{dd}, J=5.5 \mathrm{~Hz} / 1.8 \mathrm{~Hz}, 1 \mathrm{H}, 6 \mathrm{a}^{\prime}-\mathrm{H}\right), 6.17(\mathrm{~d}, J=8.4 \mathrm{~Hz}, 1 \mathrm{H}, \mathrm{NH}) .{ }^{13} \mathrm{C}$ $\operatorname{NMR}\left(\mathrm{CDCl}_{3}\right) \delta 13.5,23.2,23.8,26.2,32.5,33.2,56.8,61.9,70.4,82.8$, $84.5,85.3,110.3,116.9,144.5,154.4,162.0$.

\subsection{4. (3aR,4S,6R,6aS)-6-(7-chloro-3H-[1,2,3]triazolo[4,5-d]} pyrimidin-3-yl)-2,2-dimethyltetrahydro-3aH-cyclopenta[d][1,3] dioxol-4-ol (14c.1)

To a solution of 13c.1 $(1.0 \mathrm{~g}, 3.1 \mathrm{mmol})$ in acetic acid $(10 \mathrm{~mL})$ cooled on an ice bath was added $\mathrm{NaNO}_{2}(275 \mathrm{mg}, 4.0 \mathrm{mmol})$. The mixture was allowed to reach room temperature within $1 \mathrm{~h}$ and water $(40 \mathrm{~mL})$ was then added. The resulting mixture was extracted with ethyl acetate $(3 \times 50 \mathrm{~mL})$ and the combined organic layers were dried over $\mathrm{MgSO}_{4}$ and evaporated to give $\mathbf{1 4 c . 1}$ as an oily residue $\left(0.95 \mathrm{~g}, 92 \%\right.$ yield). ${ }^{1} \mathrm{H}$ NMR (DMSO-d $\left.d_{6}\right) \delta 1.23\left(\mathrm{~s}, 3 \mathrm{H}, \mathrm{CH}_{3}\right)$, $1.48\left(\mathrm{~s}, 3 \mathrm{H}, \mathrm{CH}_{3}\right), 2.59\left(\mathrm{~m}, 2 \mathrm{H}, 5^{\prime}-\mathrm{H}\right), 4.17\left(\mathrm{td}, J=6.1 \mathrm{~Hz} / 2.6 \mathrm{~Hz}, 1 \mathrm{H}, 4^{\prime}-\right.$ $H), 4.61\left(\mathrm{dd}, J=6.8 \mathrm{~Hz} / 2.6 \mathrm{~Hz}, 1 \mathrm{H}, 3 \mathrm{a}^{\prime}-H\right), 5.20(\mathrm{bs}, 1 \mathrm{H}, \mathrm{OH}), 5.25$ (dd, $\left.J=7.3 \mathrm{~Hz} / 3.6 \mathrm{~Hz}, 1 \mathrm{H}, 6^{\prime}-H\right), 5.42\left(\mathrm{dd}, J=6.8 \mathrm{~Hz} / 3.6 \mathrm{~Hz}, 1 \mathrm{H}, 6 \mathrm{a}^{\prime}-H\right)$, $9.11(\mathrm{~s}, 1 \mathrm{H}, 2-H) .{ }^{13} \mathrm{C}$ NMR (DMSO) $\delta 24.7,26.9,38.1,62.1,74.2,82.3$, 86.0, 112.1, 129.9, 148.4, 149.6, 155.3.
4.25. (3aR,4S,6R,6aS)-6-(5,7-dichloro-3H-[1,2,3]triazolo[4,5-d] pyrimidin-3-yl)-2,2-dimethyltetrahydro-3aH-cyclopenta[d][1,3] dioxol-4-ol (14a.1)

To a solution of 13a.1 (1.0 g, $3.0 \mathrm{mmol})$ in acetic acid $(10 \mathrm{~mL})$ cooled on an ice bath was added $\mathrm{NaNO}_{2}(275 \mathrm{mg}, 4.0 \mathrm{mmol})$. The mixture was allowed to reach room temperature within $1 \mathrm{~h}$ and water $(40 \mathrm{~mL})$ was then added. The precipitate was filtered and washed with water to give $\mathbf{1 4 a . 1}$ as a white solid $(0.61 \mathrm{~g}, 59 \%$ yield, decomposition at $\left.280{ }^{\circ} \mathrm{C}\right) .{ }^{1} \mathrm{H}$ NMR $\left(\mathrm{CDCl}_{3}\right) \delta 1.34\left(\mathrm{~s}, 3 \mathrm{H}, \mathrm{CH}_{3}\right), 1.54$ $\left(\mathrm{s}, 3 \mathrm{H}, \mathrm{CH}_{3}\right), 2.41\left(\mathrm{~d}, J=15.2 \mathrm{~Hz}, 1 \mathrm{H}, 5^{\prime}-\mathrm{Ha}\right), 2.90$ (ddd, $J=15.1 \mathrm{~Hz} /$ $\left.8.0 \mathrm{~Hz} / 5.7 \mathrm{~Hz}, 1 \mathrm{H}, 5^{\prime}-\mathrm{Hb}\right), 3.23$ (d, J = 5.2 Hz, 1H, OH), 4.46 (bs, $1 \mathrm{H}, 4^{\prime}-$ $H), 4.81\left(\mathrm{~d}, J=5.7 \mathrm{~Hz}, 1 \mathrm{H}, 3 \mathrm{a}^{\prime}-H\right), 5.17\left(\mathrm{~d}, J=5.7 \mathrm{~Hz}, 1 \mathrm{H}, 6 \mathrm{a}^{\prime}-H\right), 5.39$ $\left(\mathrm{d}, J=7.2 \mathrm{~Hz}, 1 \mathrm{H}, 6^{\prime}-H\right) .{ }^{13} \mathrm{C} \mathrm{NMR}\left(\mathrm{CDCl}_{3}\right) \delta 24.2,26.6,37.2,64.7,76.6$, 84.9, 87.3, 112.0, 133.6, 150.8, 155.6, 157.9 .

4.26. 2-(((3aR,4S,6R,6aS)-6-(5,7-dichloro-3H-[1,2,3]triazolo[4,5-d] pyrimidin-3-yl)-2,2-dimethyltetrahydro-3aH-cyclopenta[d][1,3] dioxol-4-yl)oxy)ethanol (14a.2)

To a solution of 13a.2 $(1.0 \mathrm{~g}, 2.6 \mathrm{mmol})$ in acetic acid $(10 \mathrm{~mL})$ cooled on an ice bath was added $\mathrm{NaNO}_{2}(240 \mathrm{mg}, 3.5 \mathrm{mmol})$. The mixture was allowed to reach room temperature within $1 \mathrm{~h}$ and water $(40 \mathrm{~mL})$ was then added. The resulting mixture was extracted with ethyl acetate $(3 \times 50 \mathrm{~mL})$ and the combined organic layers were dried over $\mathrm{MgSO}_{4}$ and evaporated to give 14a.2 as an oily residue $\left(0.92 \mathrm{~g}, 89 \%\right.$ yield). ${ }^{1} \mathrm{H}$ NMR (DMSO- $\left.d_{6}\right) \delta 1.27\left(\mathrm{~s}, 3 \mathrm{H}, \mathrm{CH}_{3}\right)$, $1.48\left(\mathrm{~s}, 3 \mathrm{H}, \mathrm{CH}_{3}\right), 2.44\left(\mathrm{~m}, 1 \mathrm{H}, 5^{\prime}-\mathrm{Ha}\right), 2.90(\mathrm{dt}, J=13.1 \mathrm{~Hz} / 6.0 \mathrm{~Hz}, 1 \mathrm{H}$, $\left.5^{\prime}-\mathrm{Hb}\right), 3.39-3.51\left(\mathrm{~m}, 4 \mathrm{H}, \mathrm{OCH}_{2} \mathrm{CH}_{2} \mathrm{OH}\right), 4.01\left(\mathrm{~m}, 1 \mathrm{H}, 4^{\prime}-\mathrm{H}\right), 4.32$ (bs, $12 \mathrm{H}, \mathrm{OCH}_{2} \mathrm{CH}_{2} \mathrm{OH} / \mathrm{H}_{2} \mathrm{O}$ ), 4.69 (dd, $\left.J=7.2 \mathrm{~Hz} / 2.9 \mathrm{~Hz}, 1 \mathrm{H}, 3 \mathrm{a}^{\prime}-\mathrm{H}\right), 5.01$ $\left(\mathrm{m}, 1 \mathrm{H}, 6^{\prime}-H\right), 5.17$ (dd, $\left.J=7.1 \mathrm{~Hz} / 4.7 \mathrm{~Hz}, 1 \mathrm{H}, 6 \mathrm{a}^{\prime}-H\right) .{ }^{13} \mathrm{C}$ NMR $\left(\right.$ DMSO- $d_{6}$ ) $\delta$ 24.7, 26.8, 35.8, 60.0, 61.8, 70.8, 82.0, 82.1, 83.7, 112.6, $129.3,148.3,155.4$.

\subsection{7-Chloro-3-methyl-5-(methylthio)-3H-[1,2,3]triazolo[4,5-d] pyrimidine (14b.1)}

To a solution of $\mathbf{1 3 b . 1}(1.0 \mathrm{~g}, 4.9 \mathrm{mmol})$ in acetic acid $(10 \mathrm{~mL})$ cooled on an ice bath was added $\mathrm{NaNO}_{2}(450 \mathrm{mg}, 6.5 \mathrm{mmol})$. The mixture was allowed to reach room temperature within $1 \mathrm{~h}$ and water $(40 \mathrm{~mL})$ was then added. The precipitate was filtered and washed with water to give $\mathbf{1 4 b . 1}$ as a beige solid (1.0 g, 95\% yield). ${ }^{1} \mathrm{H}$ NMR (DMSO-d $\left.d_{6}\right) \delta 2.66(\mathrm{~s}, 3 \mathrm{H}, \mathrm{SCH} 3), 4.23\left(\mathrm{~s}, 3 \mathrm{H}, \mathrm{NCH}_{3}\right) .{ }^{13} \mathrm{C}$ NMR (DMSO- $\left.d_{6}\right) \delta 14.2,33.3,131.2,150.8,151.7,170.3$.

\subsection{7-Chloro-3-ethyl-5-(methylthio)-3H-[1,2,3]triazolo[4,5-d] pyrimidine (14b.2)}

To a solution of $\mathbf{1 3 b . 2}(1.0 \mathrm{~g}, 4.6 \mathrm{mmol})$ in acetic acid $(10 \mathrm{~mL})$ cooled on an ice bath was added $\mathrm{NaNO}_{2}(415 \mathrm{mg}, 6.0 \mathrm{mmol})$. The mixture was allowed to reach room temperature within $1 \mathrm{~h}$ and water $(40 \mathrm{~mL})$ was then added. The precipitate was filtered and washed with water to give $14 \mathbf{b} .2$ as a beige solid $(0.92 \mathrm{~g}, 87 \%$ yield $)$. ${ }^{1} \mathrm{H}$ NMR (DMSO- $\left.d_{6}\right) \delta 1.56\left(\mathrm{t}, J=7.3 \mathrm{~Hz}, 3 \mathrm{H}, \mathrm{NCH}_{2} \mathrm{CH}_{3}\right), 2.65(\mathrm{~s}, 3 \mathrm{H}$, $\mathrm{SCH} 3$ ), 4.66 (q, $J=7.3 \mathrm{~Hz}, 2 \mathrm{H}, \mathrm{NCH}_{2} \mathrm{CH}_{3}$ ). ${ }^{13} \mathrm{C}$ NMR (DMSO- $\left.d_{6}\right) \delta 14.2$, $42.4,131.4,150.4,151.7,170.2$.

\subsection{7-Chloro-5-(ethylthio)-3-methyl-3H-[1,2,3]triazolo[4,5-d] pyrimidine (14b.3)}

To a solution of $\mathbf{1 3 b} . \mathbf{3}(1.0 \mathrm{~g}, 4.6 \mathrm{mmol})$ in acetic acid $(10 \mathrm{~mL})$ cooled on an ice bath was added $\mathrm{NaNO}_{2}(415 \mathrm{mg}, 6.0 \mathrm{mmol})$. The mixture was allowed to reach room temperature within $1 \mathrm{~h}$ and water $(40 \mathrm{~mL})$ was then added. The precipitate was filtered and washed with water to give $\mathbf{1 4 b . 3}$ as a beige solid ( $0.70 \mathrm{~g}, 87 \%$ yield $)$. 
${ }^{1} \mathrm{H}$ NMR (DMSO- $\left.d_{6}\right) \delta 1.39\left(\mathrm{t}, J=7.3 \mathrm{~Hz}, 3 \mathrm{H}, \mathrm{SCH}_{2} \mathrm{CH}_{3}\right.$ ), 3.25 (q, $\left.J=7.3 \mathrm{~Hz}, 2 \mathrm{H}, \mathrm{SCH}_{2} \mathrm{CH}_{3}\right), 4.22\left(\mathrm{~s}, 3 \mathrm{H}, \mathrm{NCH}_{3}\right) .{ }^{13} \mathrm{C}$ NMR (DMSO-d $)$ $\delta$ 14.0, 25.5, 33.3, 131.3, 150.8, 151.8, 169.7.

4.30. 7-Chloro-3-ethyl-5-(ethylthio)-3H-[1,2,3]triazolo[4,5-d] pyrimidine (14b.4)

To a solution of $\mathbf{1 3 b} .4(1.0 \mathrm{~g}, 4.3 \mathrm{mmol})$ in acetic acid $(10 \mathrm{~mL})$ cooled on an ice bath was added $\mathrm{NaNO}_{2}(395 \mathrm{mg}, 5.7 \mathrm{mmol})$. The mixture was allowed to reach room temperature within $1 \mathrm{~h}$ and water $(40 \mathrm{~mL})$ was then added. The precipitate was filtered and washed with water to give $\mathbf{1 4 b . 4}$ as a beige solid ( $0.88 \mathrm{~g}, 87 \%$ yield). ${ }^{1} \mathrm{H}$ NMR (DMSO- $\left.d_{6}\right) \delta 1.39\left(\mathrm{t}, J=7.3 \mathrm{~Hz}, 3 \mathrm{H}, \mathrm{SCH}_{2} \mathrm{CH}_{3}\right), 1.56(\mathrm{t}$, $\left.J=7.3 \mathrm{~Hz}, 3 \mathrm{H}, \mathrm{NCH}_{2} \mathrm{CH}_{3}\right), 3.24\left(\mathrm{q}, J=7.3 \mathrm{~Hz}, 2 \mathrm{H}, \mathrm{SCH}_{2} \mathrm{CH}_{3}\right), 4.66(\mathrm{q}$, $\left.J=7.3 \mathrm{~Hz}, 2 \mathrm{H}, \mathrm{NCH}_{2} \mathrm{CH}_{3}\right) .{ }^{13} \mathrm{C}$ NMR (DMSO-d $\left.{ }_{6}\right) \delta 13.9,14.2,25.5,42.5$, 131.5, 150.4, 151.8, 169.6.

\subsection{7-Chloro-3-methyl-5-(propylthio)-3H-[1,2,3]triazolo[4,5-d] pyrimidine (14b.5)}

To a solution of $\mathbf{1 3 b . 5}(1.0 \mathrm{~g}, 4.3 \mathrm{mmol})$ in acetic acid $(10 \mathrm{~mL})$ cooled on an ice bath was added $\mathrm{NaNO}_{2}(395 \mathrm{mg}, 5.7 \mathrm{mmol})$. The mixture was allowed to reach room temperature within $1 \mathrm{~h}$ and water $(40 \mathrm{~mL})$ was then added. The precipitate was filtered and washed with water to give $\mathbf{1 4 b . 5}$ as an orange solid $(0.87 \mathrm{~g}, 84 \%$ yield). ${ }^{1} \mathrm{H}$ NMR (DMSO- $\left.d_{6}\right) \delta 1.03\left(\mathrm{t}, J=7.1 \mathrm{~Hz}, 3 \mathrm{H}, \mathrm{SCH}_{2} \mathrm{CH}_{2} \mathrm{CH}_{3}\right.$ ), 1.76 (h, $J=7.0 \mathrm{~Hz}, 2 \mathrm{H}, \mathrm{SCH}_{2} \mathrm{CH}_{2} \mathrm{CH}_{3}$ ), 3.23 (t, $J=7.1 \mathrm{~Hz}, 2 \mathrm{H}$, $\left.\mathrm{SCH}_{2} \mathrm{CH}_{2} \mathrm{CH}_{3}\right), 4.22\left(\mathrm{~s}, 3 \mathrm{H}, \mathrm{NCH}_{3}\right) .{ }^{13} \mathrm{C}$ NMR (DMSO-d $\left.d_{6}\right) \delta 13.2,21.7$, $32.8,33.2,131.3,150.8,151.7,169.8$.

\subsection{7-Chloro-3-ethyl-5-(propylthio)-3H-[1,2,3]triazolo[4,5-d] pyrimidine (14b.6)}

To a solution of $\mathbf{1 3 b . 6}$ (1.0 g, $4.0 \mathrm{mmol})$ in acetic acid $(10 \mathrm{~mL})$ cooled on an ice bath was added $\mathrm{NaNO}_{2}(375 \mathrm{mg}, 5.4 \mathrm{mmol})$. The mixture was allowed to reach room temperature within $1 \mathrm{~h}$ and water $(40 \mathrm{~mL})$ was then added. The precipitate was filtered and washed with water to give $\mathbf{1 4 b . 6}$ as an orange solid $(0.90 \mathrm{~g}, 86 \%$ yield). ${ }^{1} \mathrm{H}$ NMR (DMSO-d $\left.{ }_{6}\right) \delta 1.02\left(\mathrm{t}, J=7.4 \mathrm{~Hz}, 3 \mathrm{H}, \mathrm{SCH}_{2} \mathrm{CH}_{2} \mathrm{CH}_{3}\right.$ ), $1.56\left(\mathrm{t}, J=7.3 \mathrm{~Hz}, 3 \mathrm{H}, \mathrm{NCH}_{2} \mathrm{CH}_{3}\right), 1.76(\mathrm{~h}, J=7.4 \mathrm{~Hz}, 2 \mathrm{H}$, $\mathrm{SCH}_{2} \mathrm{CH}_{2} \mathrm{CH}_{3}$ ), $3.24\left(\mathrm{t}, J=7.2 \mathrm{~Hz}, 2 \mathrm{H}, \mathrm{SCH}_{2} \mathrm{CH}_{2} \mathrm{CH}_{3}\right), 4.66$ (q, $J=7.3 \mathrm{~Hz}, 2 \mathrm{H}, \mathrm{NCH}_{2} \mathrm{CH}_{3}$ ). ${ }^{13} \mathrm{C}$ NMR (DMSO- $\left.d_{6}\right) \delta 13.3,14.2,21.7,32.9$, $42.5,131.5,150.3,151.8,169.7$.

\subsection{7-Chloro-3-cyclopentyl-5-(propylthio)-3H-[1,2,3]triazolo[4,5-} d]pyrimidine (14b.7)

To a solution of $\mathbf{1 3 b . 7}(1.0 \mathrm{~g}, 3.5 \mathrm{mmol})$ in acetic acid $(10 \mathrm{~mL})$ cooled on an ice bath was added $\mathrm{NaNO}_{2}(320 \mathrm{mg}$, $4.6 \mathrm{mmol})$. The mixture was allowed to reach room temperature within $1 \mathrm{~h}$ and water $(40 \mathrm{~mL})$ was then added. The precipitate was filtered and washed with water to give $\mathbf{1 4 b . 7}$ as an orange solid $(0.70 \mathrm{~g}, 67 \%$ yield). ${ }^{1} \mathrm{H}$ NMR (DMSO- $\left.d_{6}\right) \delta 1.03\left(\mathrm{t}, J=7.3 \mathrm{~Hz}, 3 \mathrm{H}, \mathrm{SCH}_{2} \mathrm{CH}_{2} \mathrm{CH}_{3}\right.$ ), $1.76\left(\mathrm{~m}, 4 \mathrm{H}, 3^{\prime}-\mathrm{Ha} / 4^{\prime}-\mathrm{Ha} / \mathrm{SCH}_{2} \mathrm{CH}_{2} \mathrm{CH}_{3}\right), 1.93\left(\mathrm{~m}, 2 \mathrm{H}, 3^{\prime}-\mathrm{Hb} / 4^{\prime}-\mathrm{Hb}\right)$, $2.24\left(\mathrm{~m}, 4 \mathrm{H}, 2^{\prime}-\mathrm{H}_{2} / 5^{\prime}-\mathrm{H}_{2}\right), 3.21\left(\mathrm{t}, J=7.1 \mathrm{~Hz}, 2 \mathrm{H}, \mathrm{SCH}_{2} \mathrm{CH}_{2} \mathrm{CH}_{3}\right), 5.35$ $\left(\mathrm{p}, J=7.1 \mathrm{~Hz}, 1 \mathrm{H}, 1^{\prime}-H\right) .{ }^{13} \mathrm{C}$ NMR (DMSO- $\left.d_{6}\right) \delta 13.2,21.8,24.3,31.8$, $32.8,59.7,131.8,150.1,151.7,169.3$.

4.34. (3aR,4S,6R,6aS)-6-(7-chloro-5-(propylthio)-3H-[1,2,3] triazolo[4,5-d]pyrimidin-3-yl)-2,2-dimethyltetrahydro-3aHcyclopenta[d][1,3]dioxol-4-ol (14b.8)

To a solution of $\mathbf{1 3 b . 8}(1.0 \mathrm{~g}, 2.7 \mathrm{mmol})$ in acetic acid $(10 \mathrm{~mL})$ cooled on an ice bath was added $\mathrm{NaNO}_{2}(250 \mathrm{mg}, 3.6 \mathrm{mmol})$. The mixture was allowed to reach room temperature within $1 \mathrm{~h}$ and water $(40 \mathrm{~mL})$ was then added. The resulting mixture was extracted with ethyl acetate $(3 \times 50 \mathrm{~mL})$ and the combined organic layers were dried over $\mathrm{MgSO}_{4}$ and evaporated to give $\mathbf{1 4 b . 8}$ as an oily residue $(0.88 \mathrm{~g}, 85 \%$ yield $) .{ }^{1} \mathrm{H}$ NMR $\left(\mathrm{CDCl}_{3}\right) \delta 1.10(\mathrm{t}, J=7.4 \mathrm{~Hz}, 3 \mathrm{H}$, $\left.\mathrm{SCH}_{2} \mathrm{CH}_{2} \mathrm{CH}_{3}\right), 1.33$ (s, 3H, $\left.\mathrm{CH}_{3}\right), 1.54\left(\mathrm{~s}, 3 \mathrm{H}, \mathrm{CH}_{3}\right), 1.83(\mathrm{~h}, J=7.3 \mathrm{~Hz}$, $\left.2 \mathrm{H}, \mathrm{SCH}_{2} \mathrm{CH}_{2} \mathrm{CH}_{3}\right), 2.38\left(\mathrm{~d}, J=15.3 \mathrm{~Hz}, 1 \mathrm{H}, 5^{\prime}-\mathrm{Ha}\right), 2.90\left(\mathrm{~m}, 1 \mathrm{H}, 5^{\prime}-\right.$ $\mathrm{Hb}$ ), 3.24 (td, $\left.J=7.1 \mathrm{~Hz} / 1.7 \mathrm{~Hz}, 2 \mathrm{H}, \mathrm{SCH}_{2} \mathrm{CH}_{2} \mathrm{CH}_{3}\right), 3.76$ (d, $J=8.8 \mathrm{~Hz}$, $1 \mathrm{H}, \mathrm{OH}), 4.44\left(\mathrm{~m}, 1 \mathrm{H}, 4^{\prime}-H\right), 4.80\left(\mathrm{~d}, J=5.6 \mathrm{~Hz}, 1 \mathrm{H}, 3 \mathrm{a}^{\prime}-\mathrm{H}\right), 5.04(\mathrm{~d}$, $\left.J=5.7 \mathrm{~Hz}, 1 \mathrm{H}, 6 \mathrm{a}^{\prime}-H\right), 5.34\left(\mathrm{~d}, J=7.7 \mathrm{~Hz}, 1 \mathrm{H}, 6^{\prime}-H\right) .{ }^{13} \mathrm{C} \mathrm{NMR}\left(\mathrm{CDCl}_{3}\right)$ $\delta$ 13.5, 22.1, 24.1, 26.6, 33.8, 37.0, 64.1, 76.7, 85.3, 87.6, 111.6, 132.0, $150.2,153.7,172.5$.

\subsection{2-(((3aR,4S,6R,6aS)-6-(7-chloro-5-(propylthio)-3H-[1,2,3] triazolo[4,5-d]pyrimidin-3-yl)-2,2-dimethyltetrahydro-3aH- cyclopenta[d][1,3]dioxol-4-yl)oxy)ethanol (14b.9)}

To a solution of $\mathbf{1 3 b . 9}$ (1.0 g, $2.4 \mathrm{mmol})$ in acetic acid $(10 \mathrm{~mL})$ cooled on an ice bath was added $\mathrm{NaNO}_{2}(225 \mathrm{mg}, 3.2 \mathrm{mmol})$. The mixture was allowed to reach room temperature within $1 \mathrm{~h}$ and water $(40 \mathrm{~mL})$ was then added. The resulting mixture was extracted with ethyl acetate $(3 \times 50 \mathrm{~mL})$ and the combined organic layers were dried over $\mathrm{MgSO}_{4}$ and evaporated to give $\mathbf{1 4 b . 9}$ as an oily residue $(0.96 \mathrm{~g}, 94 \%$ yield $) .{ }^{1} \mathrm{H}$ NMR $\left(\mathrm{CDCl}_{3}\right) \delta 1.09(\mathrm{t}, J=7.4 \mathrm{~Hz}, 3 \mathrm{H}$, $\left.\mathrm{SCH}_{2} \mathrm{CH}_{2} \mathrm{CH}_{3}\right), 1.37\left(\mathrm{~s}, 3 \mathrm{H}, \mathrm{CH}_{3}\right), 1.55\left(\mathrm{~s}, 3 \mathrm{H}, \mathrm{CH}_{3}\right), 1.83(\mathrm{~h}, J=7.4 \mathrm{~Hz}$, $\left.2 \mathrm{H}, \mathrm{SCH}_{2} \mathrm{CH}_{2} \mathrm{CH}_{3}\right), 2.14(\mathrm{t}, J=6.0 \mathrm{~Hz}, 1 \mathrm{H}, \mathrm{OH}), 2.54\left(\mathrm{~m}, 1 \mathrm{H}, 5^{\prime}-\mathrm{Ha}\right)$, $2.70\left(\mathrm{~m}, 1 \mathrm{H}, 5^{\prime}-\mathrm{Hb}\right), 3.21\left(\mathrm{t}, J=7.2 \mathrm{~Hz}, 2 \mathrm{H}, \mathrm{SCH}_{2} \mathrm{CH}_{2} \mathrm{CH}_{3}\right), 3.49-3.65$ (m, $\left.4 \mathrm{H}, \mathrm{OCH}_{2} \mathrm{CH}_{2} \mathrm{OH}\right), 4.05\left(\mathrm{~m}, 1 \mathrm{H}, 4^{\prime}-\mathrm{H}\right), 4.88\left(\mathrm{~d}, J=6.3 \mathrm{~Hz}, 1 \mathrm{H}, 3 \mathrm{a}^{\prime}-\right.$ $H), 5.21\left(\mathrm{td}, J=7.4 \mathrm{~Hz} / 6.4 \mathrm{~Hz} / 2.5 \mathrm{~Hz}, 1 \mathrm{H}, 6^{\prime}-H\right), 5.53(\mathrm{dd}, J=6.3 \mathrm{~Hz} /$ $\left.2.1 \mathrm{~Hz}, 1 \mathrm{H}, 6 \mathrm{a}^{\prime}-H\right) .{ }^{13} \mathrm{C} \mathrm{NMR}\left(\mathrm{CDCl}_{3}\right) \delta 13.6,22.3,24.5,26.8,33.9,35.9$, $61.8,63.4,70.7,82.9,83.6,84.0,112.4,132.2,150.7,153.4,171.8$.

4.36. (3aR,4S,6R,6aS)-6-(7-(((1R,2S)-2-(3,4-difluorophenyl) cyclopropyl)amino)-3H-[1,2,3]triazolo[4,5-d]pyrimidin-3-yl)-2,2dimethyltetrahydro-3aH-cyclopenta[d][1,3]dioxol-4-ol (15c.1)

The mixture of 14c.1 (0.5 g, $1.6 \mathrm{mmol}),(1 R, 2 S)-2-(3,4-$ difluorophenyl)cyclopropanamine $(0.41 \mathrm{~g}, 2.4 \mathrm{mmol})$ and triethylamine $(0.29 \mathrm{~mL}, 2.05 \mathrm{mmol})$ in acetonitrile $(10 \mathrm{~mL})$ was left to react at room temperature for $2 \mathrm{~h}$. After evaporation of the solvent, the residue was purified by column chromatography on silica gel using hexane/ethyl acetate gradient to give $\mathbf{1 5 c} .1$ as a white solid $(0.67 \mathrm{~g}$, $94 \%$ yield). ${ }^{1} \mathrm{H}$ NMR (DMSO- $\left.d_{6}\right) \delta 1.26\left(\mathrm{~s}, 3 \mathrm{H}, \mathrm{CH}_{3}\right), 1.37(\mathrm{q}, J=6.0 \mathrm{~Hz}$, $0.8 \mathrm{H}, 3^{\prime}-\mathrm{Ha}$ major), $1.42\left(\mathrm{~m}, 0.2 \mathrm{H}, 3^{\prime}-\mathrm{Ha}\right.$ minor), $1.47\left(\mathrm{~m}, 3.2 \mathrm{H}, \mathrm{CH}_{3} /\right.$ 3'-Hb minor), 1.54 (dt, $J=9.9 \mathrm{~Hz} / 5.3 \mathrm{~Hz}, 0.8 \mathrm{H}, 3^{\prime}-\mathrm{Hb}$ major), 2.18 (ddd, $J=9.4 \mathrm{~Hz} / 6.3 \mathrm{~Hz} / 3.3 \mathrm{~Hz}, 0.8 \mathrm{H}, 2^{\prime}-H$ major), $2.26\left(\mathrm{~m}, 0.2 \mathrm{H}, 2^{\prime}-H\right.$ minor), 2.45-2.60 (m, 2H, 5'"'-H), 3.28 (dd, $J=7.7 \mathrm{~Hz} / 3.6 \mathrm{~Hz}, 0.8 \mathrm{H}$, $1^{\prime}-H$ major), $3.78\left(\mathrm{~m}, 0.2 \mathrm{H}, 1^{\prime}-H\right.$ minor), $4.14\left(\mathrm{~m}, 1 \mathrm{H}, 4^{\prime \prime \prime}-H\right), 4.57(\mathrm{dd}$, $J=7.1 \mathrm{~Hz} / 2.8 \mathrm{~Hz}, 1 \mathrm{H}, 3 \mathrm{a}$ '"- $H), 5.08$ (td, $\left.J=7.9 \mathrm{~Hz} / 4.4 \mathrm{~Hz}, 1 \mathrm{H}, 6^{\prime \prime \prime}-H\right)$, $5.22(\mathrm{~m}, 0.2 \mathrm{H}, 6 \mathrm{a}$ "'- $H$ minor), 5.29 (m, 1.8H, 6a"'-H major/OH), 7.03 $\left(\mathrm{m}, 0.2 \mathrm{H}, 2^{\prime \prime}-\mathrm{H}\right.$ minor $), 7.09\left(\mathrm{~m}, 0.8 \mathrm{H}, 6^{\prime \prime}-\mathrm{H}\right.$ major $), 7.27-7.37(\mathrm{~m}, 2 \mathrm{H}$, $2^{\prime \prime}-H / 5^{\prime \prime}-H$ ), 8.32 (s, 0.2H, 2- $H$ minor), 8.42 (s, 0.8H, 2- $H$ minor), 9.01 (d, $J=3.7 \mathrm{~Hz}, 0.2 \mathrm{H}, \mathrm{NH}$ minor), $9.34(\mathrm{~d}, J=4.0 \mathrm{~Hz}, 0.8 \mathrm{H}, \mathrm{NH}$ major). ${ }^{13} \mathrm{C}$ NMR (DMSO- $d_{6}$ ) $\delta 15.2,23.7,24.7,26.9,33.9,37.9,62.1,74.3$, $82.2,86.0,112.0,114.8,117.1,122.9,124.6,139.2,146.8,148.1,148.4$, $148.7,150.3,155.0,156.4,156.5$.

4.37. (3aR,4S,6R,6aS)-6-(7-amino-5-chloro-3H-[1,2,3]triazolo[4,5d]pyrimidin-3-yl)-2,2-dimethyltetrahydro-3aH-cyclopenta[d][1,3] dioxol-4-ol (15a.1)

The solution of 14a.1 (0.5 g, $1.45 \mathrm{mmol})$ in THF (10 mL) was saturated with ammonia gas in a sealed vessel and left to react at room temperature for $1 \mathrm{~h}$. After evaporation of the solvent, the residue was purified by column chromatography on silica gel using hexane/ethyl acetate gradient to give $\mathbf{1 5 a . 1}$ as a white solid (0.45 g, 
95\% yield). ${ }^{1} \mathrm{H}$ NMR (DMSO- $\left.d_{6}\right) \delta 1.26\left(\mathrm{~s}, 3 \mathrm{H}, \mathrm{CH}_{3}\right), 1.47\left(\mathrm{~s}, 3 \mathrm{H}, \mathrm{CH}_{3}\right)$, $2.42\left(\mathrm{~m}, 1 \mathrm{H}, 5^{\prime}-\mathrm{Ha}\right), 2.55\left(\mathrm{~m}, 1 \mathrm{H}, 5^{\prime}-\mathrm{Hb}\right), 4.13\left(\mathrm{~m}, 1 \mathrm{H}, 4^{\prime}-\mathrm{H}\right), 4.56(\mathrm{dd}$, $\left.J=7.0 \mathrm{~Hz} / 3.0 \mathrm{~Hz}, 1 \mathrm{H}, 3 \mathrm{a}^{\prime}-H\right), 4.98$ (ddd, $J=8.6 \mathrm{~Hz} / 7.0 \mathrm{~Hz} / 4.2 \mathrm{~Hz}, 1 \mathrm{H}$, $\left.6^{\prime}-H\right), 5.25$ (m, 2H, OH/6a'-H), 8.64 (bs, $\left.1 \mathrm{H}, \mathrm{NHa}\right), 8.96$ (bs, $\left.1 \mathrm{H}, \mathrm{NHb}\right)$. ${ }^{13} \mathrm{C}$ NMR (DMSO- $\left.d_{6}\right) \delta 24.7,26.8,37.8,61.9,74.2,82.1,85.9,112.0$, $123.6,150.0,156.5,157.6$

4.38. (3aR,4S,6R,6aS)-6-(5-chloro-7-(((1R,2S)-2-(3,4difluorophenyl)cyclopropyl)amino)-3H-[1,2,3]triazolo[4,5-d] pyrimidin-3-yl)-2,2-dimethyltetrahydro-3aH-cyclopenta[d][1,3] dioxol-4-ol (15a.2)

The mixture of 14a.1 (0.5 g, $1.45 \mathrm{mmol}),(1 R, 2 S)-2-(3,4-$ difluorophenyl)cyclopropanamine $(0.37 \mathrm{~g}, 2.2 \mathrm{mmol})$ and triethylamine $(0.26 \mathrm{~mL}, 1.86 \mathrm{mmol})$ in acetonitrile $(10 \mathrm{~mL})$ was left to react at room temperature for 30 min. After evaporation of the solvent, the residue was purified by column chromatography on silica gel using hexane/ethyl acetate gradient to give 15a.2 as a white solid (0.59 g, 85\% yield, decomposition at $85{ }^{\circ} \mathrm{C}$ ). ${ }^{1} \mathrm{H}$ NMR (DMSO- $d_{6}$ ) $\delta 1.25$ (s, $0.9 \mathrm{H}, \mathrm{CH}_{3}$ minor), $1.26\left(\mathrm{~s}, 2.1 \mathrm{H}, \mathrm{CH}_{3}\right.$ major), $1.41-1.50(\mathrm{~m}$, $5 \mathrm{H}, \mathrm{CH}_{3} / 3^{\prime}-\mathrm{H}_{2}$ ), 2.23 (ddd, $J=9.7 \mathrm{~Hz} / 6.6 \mathrm{~Hz} / 3.3 \mathrm{~Hz}, 0.7 \mathrm{H}, 2^{\prime}-\mathrm{H}$ major), 2.29 (ddd, $J=9.5 \mathrm{~Hz} / 6.5 \mathrm{~Hz} / 3.2 \mathrm{~Hz}, 0.3 \mathrm{H}, 2^{\prime}-\mathrm{H}$ minor), $2.35-2.44\left(\mathrm{~m}, 1 \mathrm{H}, 5^{\prime \prime \prime}-\mathrm{Ha}\right), 2.56\left(\mathrm{~m}, 1 \mathrm{H}, 5^{\prime \prime \prime}-\mathrm{Hb}\right), 3.13(\mathrm{dt}, J=7.7 \mathrm{~Hz}$ $3.9 \mathrm{~Hz}, 0 ., 1^{\prime}-\mathrm{H}$ major), 3.79 ( $\mathrm{m}, 0.3 \mathrm{H}, 1^{\prime}-\mathrm{H}$ minor), $4.14\left(\mathrm{~m}, 1 \mathrm{H}, 4^{\prime \prime \prime}-\right.$ $H$ ), 4.54 (dd, $J=7.0 \mathrm{~Hz} / 2.8 \mathrm{~Hz}, 0.3 \mathrm{H}, 3 \mathrm{a}$ '"- $H$ minor), 4.57 (dd, $J=7.0 \mathrm{~Hz} / 2.8 \mathrm{~Hz}, 0.7 \mathrm{H}, 3 \mathrm{a}$ "'- $H$ major), 4.97 (td, $J=8.3 \mathrm{~Hz} / 4.3 \mathrm{~Hz}$ $0.3 \mathrm{H}, 6^{\prime \prime \prime}-H$ minor), 5.00 (td, $J=7.9 \mathrm{~Hz} / 4.1 \mathrm{~Hz}, 0.7 \mathrm{H}, 6^{\prime \prime \prime}-H$ major), 5.21 (dd, $J=6.9 \mathrm{~Hz} / 4.2 \mathrm{~Hz}, 0.3 \mathrm{H}, 6 \mathrm{a}$ "'- $H$ minor), 5.23 (d, $J=4.6 \mathrm{~Hz}$, $1 \mathrm{H}, \mathrm{OH}$ ), 5.27 (dd, $J=6.9 \mathrm{~Hz} / 4.1 \mathrm{~Hz}, 0.7 \mathrm{H}, 6 \mathrm{a}$ '" $-H$ major), 7.03 (bs, $0.3 \mathrm{H}, 6^{\prime \prime}-\mathrm{H}$ minor), 7.14 (bs, $0.7 \mathrm{H}, 6^{\prime \prime}-H$ major), 7.25 (m, 0.3H, 2" $-\mathrm{H}$ minor), 7.31-7.37 (m, $\left.1 \mathrm{H}, 5^{\prime \prime}-H\right), 7.40\left(\mathrm{~m}, 0.7 \mathrm{H}, 2^{\prime \prime}-\mathrm{H}\right.$ major), 9.55 (bs, $0.3 \mathrm{H}, \mathrm{NH}$ minor), 9.81 (bs, $0.7 \mathrm{H}, \mathrm{NH}$ major). ${ }^{13} \mathrm{C}$ NMR (DMSO-d $\left.\mathrm{d}_{6}\right)$ $\delta$ 14.5, 23.7, 24.7, 26.9, 33.6, 37.9, 62.2, 74.3, 82.2, 86.0, 112.0, 117.1, $123.4,124.1,138.6,146.9,148.4,149.5,150.4,151.1,155.4,156.2$, 156.8, 157.7.

4.39. $2-(((3 a R, 4 S, 6 R, 6 a S)-6-(5-c h l o r o-7-(((1 R, 2 S)-2-(3,4-$ difluorophenyl)cyclopropyl)amino)-3H-[1,2,3]triazolo[4,5-d] pyrimidin-3-yl)-2,2-dimethyltetrahydro-3aH-cyclopenta[d][1,3] dioxol-4-yl)oxy)ethanol (15a.3)

The mixture of 14a.2 (0.5 g, $1.28 \mathrm{mmol}),(1 R, 2 S)-2-(3,4-$ difluorophenyl)cyclopropanamine $(0.33 \mathrm{~g}, 1.9 \mathrm{mmol})$ and triethylamine $(0.23 \mathrm{~mL}, 1.64 \mathrm{mmol})$ in acetonitrile $(10 \mathrm{~mL})$ was left to react at room temperature for $30 \mathrm{~min}$. After evaporation of the solvent, the residue was purified by column chromatography on silica gel using hexane/ethyl acetate gradient to give 15a.3 as a yellowish solid $\left(0.62 \mathrm{~g}, 92 \%\right.$ yield). ${ }^{1} \mathrm{H}$ NMR (DMSO- $\left.d_{6}\right) \delta 1.26\left(\mathrm{~s}, 0.9 \mathrm{H}, \mathrm{CH}_{3}\right.$ minor), 1.27 (s, $2.1 \mathrm{H}, \mathrm{CH}_{3}$ major), $1.42-1.50\left(\mathrm{~m}, 5 \mathrm{H}, \mathrm{CH}_{3} / 3^{\prime}-\mathrm{H}_{2}\right), 2.24$ (ddd, $J=9.7 \mathrm{~Hz} / 6.5 \mathrm{~Hz} / 3.4 \mathrm{~Hz}, 0.7 \mathrm{H}, 2^{\prime}-H$ major), 2.29 (ddd, $J=9.6 \mathrm{~Hz} / 6.5 \mathrm{~Hz} / 3.2 \mathrm{~Hz}, 0.3 \mathrm{H}, 2^{\prime}-\mathrm{H}$ minor $), 2.5\left(\mathrm{~m}, 1 \mathrm{H}, 5^{\prime \prime \prime}-\mathrm{Ha}\right), 2.66$ $\left(\mathrm{m}, 1 \mathrm{H}, 5^{\prime \prime \prime}-\mathrm{Hb}\right), 3.14\left(\mathrm{dt}, J=7.8 \mathrm{~Hz} / 3.9 \mathrm{~Hz}, 0.7 \mathrm{H}, 1^{\prime}-\mathrm{H}\right.$ major$)$, 3.38-3.51 (m, $\left.4 \mathrm{H}, \mathrm{OCH}_{2} \mathrm{CH}_{2} \mathrm{OH}\right), 3.77\left(\mathrm{~m}, 0.3 \mathrm{H}, 1^{\prime}-\mathrm{H}\right.$ minor), 4.00 $\left(\mathrm{m}, 1 \mathrm{H}, 4^{\prime \prime \prime}-H\right), 4.56(\mathrm{~m}, 1 \mathrm{H}, \mathrm{OH}), 4.67(\mathrm{dd}, J=7.3 \mathrm{~Hz} / 3.1 \mathrm{~Hz}, 0.3 \mathrm{H}$, 3a"'- $H$ minor), 4.70 (dd, $J=7.3 \mathrm{~Hz} / 3.1 \mathrm{~Hz}, 0.7 \mathrm{H}, 3 \mathrm{a}$ '"- $H$ major), 5.01 (ddd, $J=10.1 \mathrm{~Hz} / 6.8 \mathrm{~Hz} / 5.0 \mathrm{~Hz}, 0.3 \mathrm{H}, 6^{\prime \prime \prime}-H$ minor), 5.04 (ddd, $J=9.7 \mathrm{~Hz} / 6.8 \mathrm{~Hz} / 4.7 \mathrm{~Hz}, 0.7 \mathrm{H}, 6^{\prime \prime \prime}-H$ major), $5.17(\mathrm{dd}, J=7.3 \mathrm{~Hz} /$ $4.8 \mathrm{~Hz}, 0.3 \mathrm{H}, 6 \mathrm{a}$ '”- $H$ minor), 5.22 (dd, $J=7.3 \mathrm{~Hz} / 4.6 \mathrm{~Hz}, 0.7 \mathrm{H}, 6 \mathrm{a}$ '”- $H$ major), $7.03\left(\mathrm{~d}, J=8.3 \mathrm{~Hz}, 0.3 \mathrm{H}, 6^{\prime \prime}-H\right.$ minor), $7.14(\mathrm{~d}, J=8.3 \mathrm{~Hz}$, $0.7 \mathrm{H}, 6^{\prime \prime}-H$ major), 7.25 (ddd, $J=12.0 \mathrm{~Hz} / 7.8 \mathrm{~Hz} / 2.0 \mathrm{~Hz}, 0.3 \mathrm{H}, 2^{\prime \prime}-H$ minor), 7.31-7.37 (m, $\left.1 \mathrm{H}, 5^{\prime \prime}-H\right), 7.40$ (ddd, $J=12.0 \mathrm{~Hz} / 7.8 \mathrm{~Hz} / 2.0 \mathrm{~Hz}$, $0.7 \mathrm{H}, 2^{\prime \prime}-H$ major), 9.56 (d, $J=4.9 \mathrm{~Hz}, 0.3 \mathrm{H}, \mathrm{NH}$ minor), 9.83 (d, $J=3.4 \mathrm{~Hz}, 0.7 \mathrm{H}, \mathrm{NH}$ major). ${ }^{13} \mathrm{C}$ NMR (DMSO-d 6 ) $\delta 14.5,23.7,24.7$, 26.8, 33.6, 35.5, 60.0, 61.7, 70.7, 81.9, 83.7, 112.5, 114.9, 115.6, 117.0, $123.4,124.1,138.6,147.2,148.6,149.5,150.0,151.2,155.4,156.6$,
156.9, 157.8 .

4.40. $N$-((1R,2S)-2-(3,4-difluorophenyl)cyclopropyl)-3-methyl-5(methylthio)-3H-[1,2,3]triazolo[4,5-d]pyrimidin-7-amine (7a)

The mixture of 14b.1 (0.5 g, $2.3 \mathrm{mmol}),(1 R, 2 S)-2-(3,4-$ difluorophenyl)cyclopropanamine $(0.59 \mathrm{~g}, 3.45 \mathrm{mmol})$ and triethylamine $(0.42 \mathrm{~mL}, 2.95 \mathrm{mmol})$ in acetonitrile $(10 \mathrm{~mL})$ was left to react at room temperature for $2 \mathrm{~h}$. After evaporation of the solvent, the residue was purified by column chromatography on silica gel using hexane/ethyl acetate gradient to give $\mathbf{7 a}$ as a white solid (0.69 g, 86\% yield, m.p.: $213-216{ }^{\circ} \mathrm{C}$ ). ${ }^{1} \mathrm{H}$ NMR (DMSO- $\left.d_{6}\right) \delta 1.37$ (q, $J=6.2 \mathrm{~Hz}, 0.8 \mathrm{H}, 3^{\prime}-\mathrm{Ha}$ major $), 1.44\left(\mathrm{~m}, 0.4 \mathrm{H}, 3^{\prime}-\mathrm{H}\right.$ minor $), 1.52(\mathrm{~m}$, $0.8 \mathrm{H}, 3^{\prime}-H b$ major), 2.14 (ddd, $J=9.5 \mathrm{~Hz} / 6.3 \mathrm{~Hz} / 3.3 \mathrm{~Hz}, 0.8 \mathrm{H}, 2^{\prime}-H$ major), 2.23 ( $\mathrm{m}, 0.2 \mathrm{H}, 2^{\prime}-H$ minor), 2.34 (s, $2.4 \mathrm{H}, \mathrm{SCH} 3$ major), 2.52 (s, 0.6H, SCH 3 minor), 3.15 ( $\mathrm{m}, 0.8 \mathrm{H}, 1^{\prime}-\mathrm{H}$ major), $3.76\left(\mathrm{~m}, 0.2 \mathrm{H}, 1^{\prime}-\mathrm{H}\right.$ minor), 4.04 (s, $0.6 \mathrm{H}, \mathrm{NCH}_{3}$ minor), $4.06\left(\mathrm{~s}, 2.4 \mathrm{H}, \mathrm{NCH}_{3}\right.$ major), 7.02 (m, 0.2H, 6" $-\mathrm{H}$ minor), 7.09 ( $\mathrm{m}, 0.8 \mathrm{H}, 6^{\prime \prime}-\mathrm{H}$ major), $7.22-7.36(\mathrm{~m}, 2 \mathrm{H}$, $\left.2^{\prime \prime}-H / 5^{\prime \prime}-H\right), 8.96$ (d, $J=4.6 \mathrm{~Hz}, 0.2 \mathrm{H}, \mathrm{NH}$ minor), $9.34(\mathrm{~d}, J=3.6 \mathrm{~Hz}$, $0.8 \mathrm{H}, \mathrm{NH}$ major). ${ }^{13} \mathrm{C}$ NMR (DMSO- $\left.d_{6}\right) \delta 13.6,15.0,24.0,33.0,33.9$, $115.0,117.0,122.6,122.9,139.2,146.8,148.4,148.7,148.9,150.3$, 153.9, 169.8. Anal. $\left(\mathrm{C}_{15} \mathrm{H}_{14} \mathrm{~F}_{2} \mathrm{~N}_{6} \mathrm{~S}\right)$ theoretical: C, 51.71; H, 4.05; N, 24.12; S, 9.20. Found: C, 51.86; H, 4.27; N, 24.33, S, 9.13.

\subsection{1. $N$-((1R,2S)-2-(3,4-difluorophenyl)cyclopropyl)-3-ethyl-5- (methylthio)-3H-[1,2,3]triazolo[4,5-d]pyrimidin-7-amine (7b)}

The mixture of $\mathbf{1 4 b . 2}(0.5 \mathrm{~g}, 2.2 \mathrm{mmol}),(1 R, 2 S)-2-(3,4-$ difluorophenyl)cyclopropanamine $(0.56 \mathrm{~g}, 3.3 \mathrm{mmol})$ and triethylamine $(0.4 \mathrm{~mL}, 2.8 \mathrm{mmol})$ in acetonitrile $(10 \mathrm{~mL})$ was left to react at room temperature for $2 \mathrm{~h}$. After evaporation of the solvent, the residue was purified by column chromatography on silica gel using hexane/ethyl acetate gradient to give $\mathbf{7 b}$ as a white solid $(0.58 \mathrm{~g}$, 73\% yield, m.p.: $164-165{ }^{\circ} \mathrm{C}$ ). ${ }^{1} \mathrm{H}$ NMR (DMSO- $d_{6}$ ) $\delta 1.37$ (q, $\left.J=6.2 \mathrm{~Hz}, 1 \mathrm{H}, 3^{\prime}-\mathrm{Ha}\right), 1.49\left(\mathrm{~m}, 4 \mathrm{H}, 3^{\prime}-\mathrm{Hb} / \mathrm{NCH}_{2} \mathrm{CH}_{3}\right), 2.13$ (ddd, $J=9.4 \mathrm{~Hz} / 6.3 \mathrm{~Hz} / 3.3 \mathrm{~Hz}, 0.8 \mathrm{H}, 2^{\prime}-\mathrm{H}$ major), $2.25\left(\mathrm{~m}, 0.2 \mathrm{H}, 2^{\prime}-\mathrm{H}\right.$ minor), 2.33 (s, 2.4H, SCH 3 major), 2.52 (s, 0.6H, SCH 3 minor), 3.14 ( $\mathrm{m}, 0.8 \mathrm{H}, 1^{\prime}-\mathrm{H}$ major), $3.78\left(\mathrm{~m}, 0.2 \mathrm{H}, 1^{\prime}-H\right.$ minor), $4.49(\mathrm{q}, J=7.3 \mathrm{~Hz}$, $\left.2 \mathrm{H}, \mathrm{NCH}_{2} \mathrm{CH}_{3}\right), 7.02\left(\mathrm{~m}, 0.2 \mathrm{H}, 6^{\prime \prime}-\mathrm{H}\right.$ minor $), 7.09\left(\mathrm{~m}, 0.8 \mathrm{H}, 6^{\prime \prime}-\mathrm{H}\right.$ major), 7.22-7.36 (m, 2H, 2" $\left.-H / 5^{\prime \prime}-H\right), 8.95$ (d, $J=4.6 \mathrm{~Hz}, 0.2 \mathrm{H}, \mathrm{NH}$ minor), 9.35 (d, $J=3.7 \mathrm{~Hz}, 0.8 \mathrm{H}, \mathrm{NH}$ major). ${ }^{13} \mathrm{C}$ NMR (DMSO-d $\mathrm{d}_{6}$ ) $\delta$ 13.6, 14.5, 15.0, 24.0, 33.9, 41.3, 115.0, 117.0, 122.8, 123.0, 139.2, 146.8, 148.3, 148.7, 148.8, 150.3, 153.9, 169.8. Anal. $\left(\mathrm{C}_{16} \mathrm{H}_{16} \mathrm{~F}_{2} \mathrm{~N}_{6} \mathrm{~S}\right)$ theoretical: C, 53.03; H, 4.45; N, 23.19; S, 8.85. Found: C, 52.94; H, $4.75 ; \mathrm{N}, 23.30, \mathrm{~S}, 8.64$.

\subsection{2. $N$-((1R,2S)-2-(3,4-difluorophenyl)cyclopropyl)-5-(ethylthio)- 3-methyl-3H-[1,2,3]triazolo[4,5-d]pyrimidin-7-amine (7c)}

The mixture of $\mathbf{1 4 b . 3}$ (0.5 g, $2.2 \mathrm{mmol}),(1 R, 2 S)-2-(3,4-$ difluorophenyl)cyclopropanamine $(0.56 \mathrm{~g}, 3.3 \mathrm{mmol})$ and triethylamine $(0.4 \mathrm{~mL}, 2.8 \mathrm{mmol})$ in acetonitrile $(10 \mathrm{~mL})$ was left to react at room temperature for $2 \mathrm{~h}$. After evaporation of the solvent, the residue was purified by column chromatography on silica gel using hexane/ethyl acetate gradient to give $7 \mathbf{c}$ as a white solid $(0.45 \mathrm{~g}, 58 \%$ yield, m.p.: $210-212{ }^{\circ} \mathrm{C}$ ). ${ }^{1} \mathrm{H}$ NMR (DMSO- $\left.d_{6}\right) \delta 1.09$ (t, $J=7.3 \mathrm{~Hz}$, $2.5 \mathrm{~Hz}, \mathrm{SCH}_{2} \mathrm{CH}_{3}$ major), 1.35 (m, $1.4 \mathrm{H}, 3^{\prime}-\mathrm{Ha}$ major $/ \mathrm{SCH}_{2} \mathrm{CH}_{3}$ minor), $1.44\left(\mathrm{~m}, 0.4 \mathrm{H}, 3^{\prime}-\mathrm{H}\right.$ minor), 1.56 ( $\mathrm{m}, 0.8 \mathrm{H}, 3^{\prime}-\mathrm{Hb}$ major), 2.13 (ddd, $J=9.5 \mathrm{~Hz} / 6.3 \mathrm{~Hz} / 3.3 \mathrm{~Hz}, 0.8 \mathrm{H}, 2^{\prime}-\mathrm{H}$ major $), 2.24\left(\mathrm{~m}, 0.2 \mathrm{H}, 2^{\prime}-\mathrm{H}\right.$ minor), $2.89\left(\mathrm{~m}, 1.6 \mathrm{H}, \mathrm{SCH}_{2} \mathrm{CH}_{3}\right.$ major), $3.12\left(\mathrm{~m}, 0.4 \mathrm{H}, \mathrm{SCH}_{2} \mathrm{CH}_{3}\right.$ minor), $3.17\left(\mathrm{~m}, 0.8 \mathrm{H}, 1^{\prime}-\mathrm{H}\right.$ major), $3.75\left(\mathrm{~m}, 0.2 \mathrm{H}, 1^{\prime}-\mathrm{H}\right.$ minor), 4.03 (s, 0.6H, NCH minor), 4.05 (s, 2.4H, $\mathrm{NCH}_{3}$ major), $7.01\left(\mathrm{~m}, 0.2 \mathrm{H}, 6^{\prime \prime}-\right.$ $H$ minor), 7.07 ( $\mathrm{m}, 0.8 \mathrm{H}, 6^{\prime \prime}-H$ major), $7.21-7.36$ ( $\left.\mathrm{m}, 2 \mathrm{H}, 2^{\prime \prime}-H / 5^{\prime \prime}-H\right)$, $8.96(\mathrm{~d}, J=4.7 \mathrm{~Hz}, 0.2 \mathrm{H}, \mathrm{NH}$ minor), 9.36 (d, $J=3.8 \mathrm{~Hz}, 0.8 \mathrm{H}, \mathrm{NH}$ major). ${ }^{13} \mathrm{C}$ NMR (DMSO- $\left.d_{6}\right) \delta 14.6,15.2,24.0,24.8,32.4,34.1,114.7$, 
117.0, 122.7, 122.8, 139.4, 147.0, 148.4, 148.7, 149.4, 150.0, 153.9, 169.3. Anal. $\left(\mathrm{C}_{16} \mathrm{H}_{16} \mathrm{~F}_{2} \mathrm{~N}_{6} \mathrm{~S}\right)$ theoretical: $\mathrm{C}, 53.03 ; \mathrm{H}, 4.45 ; \mathrm{N}, 23.19 ; \mathrm{S}$, 8.85. Found: C, 53.05; H, 4.65; N, 23.27, S, 8.41.

4.43. $N-((1 R, 2 S)-2-(3,4-d i f l u o r o p h e n y l) c y c l o p r o p y l)-5-(e t h y l t h i o)-$ 3-ethyl-3H-[1,2,3]triazolo[4,5-d]pyrimidin-7-amine (7d)

The mixture of $\mathbf{1 4 b . 4}(0.5 \mathrm{~g}, 2.05 \mathrm{mmol}),(1 R, 2 S)-2-(3,4-$ difluorophenyl)cyclopropanamine $(0.52 \mathrm{~g}, 3.1 \mathrm{mmol})$ and triethylamine $(0.37 \mathrm{~mL}, 2.6 \mathrm{mmol})$ in acetonitrile $(10 \mathrm{~mL})$ was left to react at room temperature for $2 \mathrm{~h}$. After evaporation of the solvent, the residue was purified by column chromatography on silica gel using hexane/ethyl acetate gradient to give $7 \mathbf{d}$ as a white solid $(0.69 \mathrm{~g}$, 90\% yield, m.p.: $145-146.5{ }^{\circ} \mathrm{C}$ ). ${ }^{1} \mathrm{H}$ NMR (DMSO- $\left.d_{6}\right) \delta 1.09(\mathrm{t}$, $J=7.3 \mathrm{~Hz}, 2.4 \mathrm{H}, \mathrm{SCH}_{2} \mathrm{CH}_{3}$ major), 1.35 (m, $1.4 \mathrm{H}, 3^{\prime}-\mathrm{Ha}$ major/ $\mathrm{SCH}_{2} \mathrm{CH}_{3}$ minor), $1.44\left(\mathrm{~m}, 0.2 \mathrm{H}, 3^{\prime}-\mathrm{Ha}\right.$ minor), $1.47(\mathrm{t}, J=7.3 \mathrm{~Hz}$, $3.2 \mathrm{H}, \mathrm{NCH}_{2} \mathrm{CH}_{3} / 3^{\prime}-\mathrm{Hb}$ minor), 1.56 ( $\mathrm{m}, 0.8 \mathrm{H}, 3^{\prime}-\mathrm{Hb}$ major), 2.12 (ddd, $J=9.5 \mathrm{~Hz} / 6.3 \mathrm{~Hz} / 3.3 \mathrm{~Hz}, 0.8 \mathrm{H}, 2^{\prime}-H$ major $), 2.24\left(\mathrm{~m}, 0.2 \mathrm{H}, 2^{\prime}-H\right.$ minor), $2.88\left(\mathrm{~m}, 1.6 \mathrm{H}, \mathrm{SCH}_{2} \mathrm{CH}_{3}\right.$ major), $3.11\left(\mathrm{~m}, 0.4 \mathrm{H}, \mathrm{SCH}_{2} \mathrm{CH}_{3}\right.$ minor), $3.16\left(\mathrm{~m}, 0.8 \mathrm{H}, 1^{\prime}-\mathrm{H}\right.$ major), $3.77\left(\mathrm{~m}, 0.2 \mathrm{H}, 1^{\prime}-\mathrm{H}\right.$ minor), 4.48 $\left(\mathrm{q}, J=7.3 \mathrm{~Hz}, 2 \mathrm{H}, \mathrm{NCH}_{2} \mathrm{CH}_{3}\right), 7.02\left(\mathrm{~m}, 0.2 \mathrm{H}, 6^{\prime \prime}-\mathrm{H}\right.$ minor), $7.07(\mathrm{~m}$, $0.8 \mathrm{H}, 6^{\prime \prime}-H$ major), $7.23-7.36\left(\mathrm{~m}, 2 \mathrm{H}, 2^{\prime \prime}-H / 5^{\prime \prime}-H\right), 8.96$ (d, $J=3.9 \mathrm{~Hz}$, $0.2 \mathrm{H}, \mathrm{NH}$ minor), 9.37 (s, 0.8H, NH major). ${ }^{13} \mathrm{C}$ NMR (DMSO- $d_{6}$ ) $\delta 14.5,14.6,15.2,24.0,24.8,34.1,41.3,114.8,117.0,122.8,122.9,139.4$, 147.0, 148.4, 148.7, 148.9, 150.0, 154.0, 169.2. Anal. $\left(\mathrm{C}_{17} \mathrm{H}_{18} \mathrm{~F}_{2} \mathrm{~N}_{6} \mathrm{~S}\right)$ theoretical: C, 54.24; H, 4.82; N, 22.33; S, 8.52. Found: C, 54.25; H, $5.13 ; \mathrm{N}, 22.48, \mathrm{~S}, 8.43$.

4.44. $\mathrm{N}-((1 R, 2 S)-2-(3,4-d i f l u o r o p h e n y l) c y c l o p r o p y l)-3-m e t h y l-5-$ (propylthio)-3H-[1,2,3]triazolo[4,5-d]pyrimidin-7-amine (7e)

The mixture of $\mathbf{1 4 b . 5}$ (0.5 g, $2.05 \mathrm{mmol}),(1 R, 2 S)-2-(3,4-$ difluorophenyl)cyclopropanamine $(0.52 \mathrm{~g}, 3.1 \mathrm{mmol})$ and triethylamine $(0.37 \mathrm{~mL}, 2.6 \mathrm{mmol})$ in acetonitrile $(10 \mathrm{~mL})$ was heated at $50{ }^{\circ} \mathrm{C}$ for $2 \mathrm{~h}$. After evaporation of the solvent, the residue was purified by column chromatography on silica gel using hexane/ ethyl acetate gradient to give $7 \mathbf{e}$ as a white solid $(0.53 \mathrm{~g}, 69 \%$ yield, m.p.: $\left.185-187{ }^{\circ} \mathrm{C}\right) .{ }^{1} \mathrm{H}$ NMR (DMSO- $\left.d_{6}\right) \delta 0.80(\mathrm{t}, J=7.4 \mathrm{~Hz}, 2.4 \mathrm{H}$, $\mathrm{SCH}_{2} \mathrm{CH}_{2} \mathrm{CH}_{3}$ major), 1.00 (t, $J=7.3 \mathrm{~Hz}, 0.6 \mathrm{H}, \mathrm{SCH}_{2} \mathrm{CH}_{2} \mathrm{CH}_{3}$ minor), 1.37 ( $\mathrm{m}, 0.8 \mathrm{H}, 3^{\prime}-\mathrm{Ha}$ major), 1.43 ( $\mathrm{m}, 0.2 \mathrm{H}, 3^{\prime}-\mathrm{Ha}$ minor), 1.49 ( $\mathrm{m}$, $1.8 \mathrm{H}, 3^{\prime}-\mathrm{Hb}$ minor $/ \mathrm{SCH}_{2} \mathrm{CH}_{2} \mathrm{CH}_{3}$ major), 1.56 ( $\mathrm{m}, 0.8 \mathrm{H}, 3^{\prime}-\mathrm{Hb}$ major), $1.70\left(\mathrm{~h}, J=7.3 \mathrm{~Hz}, 0.4 \mathrm{H}, \mathrm{SCH}_{2} \mathrm{CH}_{2} \mathrm{CH}_{3}\right.$ minor), 2.13 (ddd, $J=9.5 \mathrm{~Hz}$ ) $6.3 \mathrm{~Hz} / 3.2 \mathrm{~Hz}, 0.8 \mathrm{H}, 2^{\prime}-H$ major $), 2.23$ ( $\mathrm{m}, 0.2 \mathrm{H}, 2^{\prime}-H$ minor $), 2.88(\mathrm{~m}$, $1.6 \mathrm{H}, \mathrm{SCH}_{2} \mathrm{CH}_{2} \mathrm{CH}_{3}$ major), 3.11 (t, $J=7.2 \mathrm{~Hz}, 0.4 \mathrm{H}, \mathrm{SCH}_{2} \mathrm{CH}_{2} \mathrm{CH}_{3}$ minor), 3.17 ( $\mathrm{m}, 0.8 \mathrm{H}, 1^{\prime}-\mathrm{H}$ major), $3.75\left(\mathrm{~m}, 0.2 \mathrm{H}, 1^{\prime}-\mathrm{H}\right.$ minor), 4.03 (s, $0.6 \mathrm{H}, \mathrm{NCH}_{3}$ minor), 4.05 (s, 2.4H, $\mathrm{NCH}_{3}$ major), $7.02\left(\mathrm{~m}, 0.2 \mathrm{H}, 6^{\prime \prime}-\right.$ $H$ minor), 7.07 (m, 0.8H, 6" $-H$ major), $7.22-7.37$ (m, 2H, $2^{\prime \prime}-H / 5^{\prime \prime}-H$ ), $8.96\left(\mathrm{~s}, 0.2 \mathrm{H}, \mathrm{NH}\right.$ minor), $9.36\left(\mathrm{~s}, 0.8 \mathrm{H}, \mathrm{NH}\right.$ major). ${ }^{13} \mathrm{C} \mathrm{NMR}$ (DMSO- $d_{6}$ ) $\delta 12.9,15.1,22.2,24.0,32.3,32.4,34.2,117.0,122.6,122.7$, 139.4, 146.7, 148.4, 148.7, 149.3, 150.3, 153.8, 169.3. Anal. $\left(\mathrm{C}_{17} \mathrm{H}_{18} \mathrm{~F}_{2} \mathrm{~N}_{6} \mathrm{~S}\right)$ theoretical: $\mathrm{C}, 54.24 ; \mathrm{H}, 4.82 ; \mathrm{N}, 22.33 ; \mathrm{S}, 8.52$. Found: C, 54.25; H, 4.87; N, 22.38, S, 7.89.

4.45. $N$-((1R,2S)-2-(3,4-difluorophenyl)cyclopropyl)-3-ethyl-5(propylthio)-3H-[1,2,3]triazolo[4,5-d]pyrimidin-7-amine (7f)

The mixture of $14 \mathrm{~b} .6$ (0.5 g, $1.95 \mathrm{mmol})$, (1R,2S)-2-(3,4difluorophenyl)cyclopropanamine $(0.50 \mathrm{~g}, 2.95 \mathrm{mmol})$ and triethylamine $(0.35 \mathrm{~mL}, 2.5 \mathrm{mmol})$ in acetonitrile $(10 \mathrm{~mL})$ was heated at $50{ }^{\circ} \mathrm{C}$ for $2 \mathrm{~h}$. After evaporation of the solvent, the residue was purified by column chromatography on silica gel using hexane/ ethyl acetate gradient to give $7 f$ as a white solid $(0.45 \mathrm{~g}, 59 \%$ yield, m.p.: $136-138{ }^{\circ} \mathrm{C}$ ). ${ }^{1} \mathrm{H}$ NMR (DMSO- $\left.d_{6}\right) \delta 0.81(\mathrm{t}, J=7.4 \mathrm{~Hz}, 2.4 \mathrm{H}$, $\mathrm{SCH}_{2} \mathrm{CH}_{2} \mathrm{CH}_{3}$ major), 0.99 (t, $J=7.3 \mathrm{~Hz}, 0.6 \mathrm{H}, \mathrm{SCH}_{2} \mathrm{CH}_{2} \mathrm{CH}_{3}$ minor), 1.37 ( $\mathrm{m}, 0.8 \mathrm{H}, 3^{\prime}-\mathrm{Ha}$ major), 1.41 ( $\mathrm{m}, 0.2 \mathrm{H}, 3^{\prime}-\mathrm{Ha}$ minor), 1.49 (m,
$4.8 \mathrm{H}, 3^{\prime}-\mathrm{Hb}$ minor/ $\mathrm{SCH}_{2} \mathrm{CH}_{2} \mathrm{CH}_{3}$ major $\left./ \mathrm{NCH}_{2} \mathrm{CH}_{3}\right), 1.56$ (m, 0.8H, 3'$\mathrm{Hb}$ major), 1.70 ( $\mathrm{h}, J=7.1 \mathrm{~Hz}, 0.4 \mathrm{H}, \mathrm{SCH}_{2} \mathrm{CH}_{2} \mathrm{CH}_{3}$ minor), 2.13 (ddd, $J=9.5 \mathrm{~Hz} / 6.3 \mathrm{~Hz} / 3.3 \mathrm{~Hz}, 0.8 \mathrm{H}, 2^{\prime}-H$ major), 2.25 (ddd, $J=9.1 \mathrm{~Hz} /$ $6.2 \mathrm{~Hz} / 3.2 \mathrm{~Hz}, 0.2 \mathrm{H}, 2^{\prime}-\mathrm{H}$ minor), 2.88 ( $\mathrm{m}, 1.6 \mathrm{H}, \mathrm{SCH}_{2} \mathrm{CH}_{2} \mathrm{CH}_{3}$ major), 3.10 (t, $J=7.0 \mathrm{~Hz}, 0.4 \mathrm{H}, \mathrm{SCH}_{2} \mathrm{CH}_{2} \mathrm{CH}_{3}$ minor), $3.16\left(\mathrm{~m}, 0.8 \mathrm{H}, 1^{\prime}-\mathrm{H}\right.$ major), $3.76\left(\mathrm{~m}, 0.2 \mathrm{H}, 1^{\prime}-\mathrm{H}\right.$ minor), $4.48\left(\mathrm{q}, J=7.3 \mathrm{~Hz}, 2 \mathrm{H}, \mathrm{NCH}_{2} \mathrm{CH}_{3}\right.$ ), 7.02 ( $\mathrm{m}, 0.2 \mathrm{H}, 2^{\prime \prime}-\mathrm{H}$ minor), 7.07 ( $\mathrm{m}, 0.8 \mathrm{H}, 6^{\prime \prime}-\mathrm{H}$ major), 7.23-7.36 ( $\left.\mathrm{m}, 2 \mathrm{H}, 2^{\prime \prime}-\mathrm{H} / 5^{\prime \prime}-H\right), 8.94$ (d, $J=4.0 \mathrm{~Hz}, 0.2 \mathrm{H}, \mathrm{NH}$ minor), 9.35 (s, $0.8 \mathrm{H}, \mathrm{NH}$ major). ${ }^{13} \mathrm{C}$ NMR (DMSO- $\left.d_{6}\right) \delta 13.0,14.5,15.0,22.3,24.0$, 32.3, 34.1, 41.3, 114.8, 117.0, 122.8, 122.9, 139.4, 147.0, 148.4, 148.7, 148.8, 150.1, 153.9, 169.6. Anal. $\left(\mathrm{C}_{18} \mathrm{H}_{20} \mathrm{~F}_{2} \mathrm{~N}_{6} \mathrm{~S}\right)$ theoretical: $\mathrm{C}, 55.37$; H, 5.16; N, 21.52; S, 8.21. Found: C, 55.30; H, 5.41; N, 21.64, S, 8.03.

4.46. 3-Cyclopentyl-N-((1R,2S)-2-(3,4-difluorophenyl) cyclopropyl)-5-(propylthio)-3H-[1,2,3]triazolo[4,5-d]pyrimidin-7amine $(\mathbf{7 g})$

The mixture of $\mathbf{1 4 b . 7}$ (0.5 g, $1.67 \mathrm{mmol}),(1 R, 2 S)-2-(3,4-$ difluorophenyl)cyclopropanamine $(0.42 \mathrm{~g}, 2.5 \mathrm{mmol})$ and triethylamine $(0.3 \mathrm{~mL}, 2.1 \mathrm{mmol})$ in acetonitrile $(10 \mathrm{~mL})$ was heated at $50{ }^{\circ} \mathrm{C}$ for $2 \mathrm{~h}$. After evaporation of the solvent, the residue was purified by column chromatography on silica gel using hexane/ ethyl acetate gradient to give $\mathbf{7 g}$ as a white solid $(0.53 \mathrm{~g}, 74 \%$ yield, m.p.: $113.5-115{ }^{\circ} \mathrm{C}$ ). ${ }^{1} \mathrm{H}$ NMR (DMSO-d $\left.d_{6}\right) \delta 0.82(\mathrm{t}, J=7.2 \mathrm{~Hz}, 2.4 \mathrm{H}$, $\mathrm{SCH}_{2} \mathrm{CH}_{2} \mathrm{CH}_{3}$ major), 0.99 (t, $J=7.3 \mathrm{~Hz}, 0.6 \mathrm{H}, \mathrm{SCH}_{2} \mathrm{CH}_{2} \mathrm{CH}_{3}$ minor), 1.38 ( $\mathrm{m}, 1 \mathrm{H}, 3^{\prime}-\mathrm{Ha}$ ), $1.44-1.55$ ( $\mathrm{m}, 2.6 \mathrm{H}, 3^{\prime}-\mathrm{Hb} / \mathrm{SCH}_{2} \mathrm{CH}_{2} \mathrm{CH}_{3}$ major), $1.72\left(\mathrm{~m}, 2.4 \mathrm{H}, \mathrm{SCH}_{2} \mathrm{CH}_{2} \mathrm{CH}_{3}\right.$ minor/3"' $\left.-\mathrm{Ha} / 4^{\prime \prime \prime}-\mathrm{Ha}\right), 1.90\left(\mathrm{~m}, 2 \mathrm{H}, 3^{\prime \prime \prime}-\right.$ $\left.\mathrm{Hb} / 4^{\prime \prime \prime}-\mathrm{Hb}\right), 2.12-2.19\left(\mathrm{~m}, 5 \mathrm{H}, 2^{\prime}-\mathrm{H} / 2^{\prime \prime \prime}-\mathrm{H}_{2} / 5^{\prime \prime \prime}-\mathrm{H}_{2}\right), 2.89(\mathrm{~m}, 1.6 \mathrm{H}$, $\mathrm{SCH}_{2} \mathrm{CH}_{2} \mathrm{CH}_{3}$ major), $3.10\left(\mathrm{~m}, 0.4 \mathrm{H}, \mathrm{SCH}_{2} \mathrm{CH}_{2} \mathrm{CH}_{3}\right.$ minor), $3.14(\mathrm{~m}$, $0.8 \mathrm{H}, 1^{\prime}-\mathrm{H}$ major), 3.78 ( $\mathrm{m}, 0.2 \mathrm{H}, 1^{\prime}-H$ minor), $5.15(\mathrm{p}, J=7.2 \mathrm{~Hz}, 1 \mathrm{H}$, $\left.1^{\prime \prime \prime}-H\right), 7.02\left(\mathrm{~m}, 0.2 \mathrm{H}, 6^{\prime \prime}-H\right.$ minor), 7.08 ( $\mathrm{m}, 0.8 \mathrm{H}, 6^{\prime \prime}-\mathrm{H}$ major), $7.22-7.37$ (m, 2H, 2"-H/5"-H), 8.94 (bs, $0.2 \mathrm{H}, \mathrm{NH}$ minor), 9.33 (bs, $0.8 \mathrm{H}, \mathrm{NH}$ major). ${ }^{13} \mathrm{C}$ NMR (DMSO- $\left.d_{6}\right) \delta 13.0,15.0,22.3,24.0,24.2$, 31.8, 32.3, 34.0, 58.3, 114.8, 117.0, 122.8, 123.1, 139.3, 146.8, 148.4, 148.6, 148.7, 150.3, 153.9, 169.0. Anal. $\left(\mathrm{C}_{21} \mathrm{H}_{24} \mathrm{~F}_{2} \mathrm{~N}_{6} \mathrm{~S}\right)$ theoretical: $\mathrm{C}$, 58.59; H, 5.62; N, 19.52; S, 7.45. Found: C, 58.44; H, 5.62; N, 19.71, S, 7.49 .

4.47. (3aR,4S,6R,6aS)-6-(7-amino-5-(propylthio)-3H-[1,2,3] triazolo[4,5-d]pyrimidin-3-yl)-2,2-dimethyltetrahydro-3aHcyclopenta[d][1,3]dioxol-4-ol (16a)

The solution of $\mathbf{1 4 b . 8}(0.5 \mathrm{~g}, 1.3 \mathrm{mmol})$ in THF $(10 \mathrm{~mL})$ was saturated with ammonia gas in a sealed vessel and left to react at room temperature for $1 \mathrm{~h}$. After evaporation of the solvent, the residue was purified by column chromatography on silica gel using hexane/ethyl acetate gradient to give $\mathbf{1 6 a}$ as a white solid $(0.38 \mathrm{~g}$, $79 \%$ yield). ${ }^{1} \mathrm{H}$ NMR (DMSO- $\left.d_{6}\right) \delta 1.00(\mathrm{t}, J=7.4 \mathrm{~Hz}, 3 \mathrm{H}$, $\left.\mathrm{SCH}_{2} \mathrm{CH}_{2} \mathrm{CH}_{3}\right), 1.26\left(\mathrm{~s}, 3 \mathrm{H}, \mathrm{CH}_{3}\right), 1.47\left(\mathrm{~s}, 3 \mathrm{H}, \mathrm{CH}_{3}\right), 1.71(\mathrm{~h}, J=7.3 \mathrm{~Hz}$, $\left.2 \mathrm{H}, \mathrm{SCH}_{2} \mathrm{CH}_{2} \mathrm{CH}_{3}\right), 2.47\left(\mathrm{~m}, 1 \mathrm{H}, 5^{\prime}-\mathrm{Ha}\right), 2.56\left(\mathrm{~m}, 1 \mathrm{H}, 5^{\prime}-\mathrm{Hb}\right), 3.08(\mathrm{~m}$, $\left.2 \mathrm{H}, \mathrm{SCH}_{2} \mathrm{CH}_{2} \mathrm{CH}_{3}\right), 4.13\left(\mathrm{~m}, 1 \mathrm{H}, 4^{\prime}-\mathrm{H}\right), 4.54(\mathrm{dd}, J=7.0 \mathrm{~Hz} / 3.0 \mathrm{~Hz}, 1 \mathrm{H}$, $3 \mathrm{a}^{\prime}-H$ ), 4.99 (ddd, $J=9.1 \mathrm{~Hz} / 7.0 \mathrm{~Hz} / 4.4 \mathrm{~Hz}, 1 \mathrm{H}, 6^{\prime}-H$ ), 5.25 (dd, $\left.J=7.0 \mathrm{~Hz} / 4.4 \mathrm{~Hz}, 1 \mathrm{H}, 6 \mathrm{a}^{\prime}-H\right), 5.28(\mathrm{~d}, J=4.7 \mathrm{~Hz}, 1 \mathrm{H}, \mathrm{OH}), 8.09(\mathrm{bs}, 1 \mathrm{H}$, $\mathrm{NHa}$ ), 8.43 (bs, $1 \mathrm{H}, \mathrm{NHb}) .{ }^{13} \mathrm{C}$ NMR (DMSO- $\left.d_{6}\right) \delta 13.4,22.6,24.7,26.9$, 32.2, 37.7, 61.9, 74.2, 82.1, 86.1, 111.9, 122.8, 149.4, 155.1, 169.3.

4.48. (3aR,4S,6R,6aS)-6-(7-(((1R,2S)-2-(3,4-difluorophenyl) cyclopropyl)amino)-5-(propylthio)-3H-[1,2,3]triazolo[4,5-d] pyrimidin-3-yl)-2,2-dimethyltetrahydro-3aH-cyclopenta[d][1,3] dioxol-4-ol (16b)

The mixture of $\mathbf{1 4 b . 8}$ (0.5 g, $1.3 \mathrm{mmol})$, (1R,2S)-2-(3,4difluorophenyl)cyclopropanamine $(0.33 \mathrm{~g}, 1.95 \mathrm{mmol})$ and triethylamine $(0.23 \mathrm{~mL}, 1.6 \mathrm{mmol})$ in acetonitrile $(10 \mathrm{~mL})$ was heated at $80{ }^{\circ} \mathrm{C}$ for $2 \mathrm{~h}$. After evaporation of the solvent, the residue was 
purified by column chromatography on silica gel using hexane/ ethyl acetate gradient to give $\mathbf{1 6 b}$ as a white solid $(0.62 \mathrm{~g}, 92 \%$ yield). ${ }^{1} \mathrm{H}$ NMR (DMSO- $\left.d_{6}\right) \delta 0.84\left(\mathrm{t}, J=7.3 \mathrm{~Hz}, 2.4 \mathrm{H}, \mathrm{SCH}_{2} \mathrm{CH}_{2} \mathrm{CH}_{3}\right.$ major), $1.00\left(\mathrm{t}, J=7.3 \mathrm{~Hz}, 0.6 \mathrm{H}, \mathrm{SCH}_{2} \mathrm{CH}_{2} \mathrm{CH}_{3}\right.$ minor), $1.26(\mathrm{~s}, 3 \mathrm{H}$, $\left.\mathrm{CH}_{3}\right), 1.38\left(\mathrm{~m}, 1 \mathrm{H}, 3^{\prime}-\mathrm{Ha}\right), 1.47\left(\mathrm{~s}, 3 \mathrm{H}, \mathrm{CH}_{3}\right), 1.53\left(\mathrm{~m}, 2.6 \mathrm{H}, 3^{\prime}-\mathrm{Hb} /\right.$ $\mathrm{SCH}_{2} \mathrm{CH}_{2} \mathrm{CH}_{3}$ major), 1.71 (h, $J=7.1 \mathrm{~Hz}, 0.4 \mathrm{H}, \mathrm{SCH}_{2} \mathrm{CH}_{2} \mathrm{CH}_{3}$ minor), $2.13\left(\mathrm{~m}, 0.8 \mathrm{H}, 2^{\prime}-H\right.$ major $), 2.25\left(\mathrm{~m}, 0.2 \mathrm{H}, 2^{\prime}-\mathrm{H}\right.$ minor $), 2.45(\mathrm{~m}, 1 \mathrm{H}$, $\left.5^{\prime \prime \prime}-\mathrm{Ha}\right), 2.54\left(\mathrm{~m}, 1 \mathrm{H}, 5^{\prime \prime \prime}-\mathrm{Hb}\right), 2.88(\mathrm{dt}, J=13.6 \mathrm{~Hz} / 7.2 \mathrm{~Hz}, 0.8 \mathrm{H}, \mathrm{SCHa}$ major), 2.94 (dt, $J=14.2 \mathrm{~Hz} / 7.2 \mathrm{~Hz}, 0.8 \mathrm{H}, \mathrm{SCHb}$ major), 3.09 (m, $0.4 \mathrm{H}, \mathrm{SCH} 2 \mathrm{minor}), 3.15\left(\mathrm{~m}, 0.8 \mathrm{H}, 1^{\prime}-\mathrm{H}\right.$ major), $3.75\left(\mathrm{~m}, 0.2 \mathrm{H}, 1^{\prime}-\mathrm{H}\right.$ minor), $4.13\left(\mathrm{~m}, 1 \mathrm{H}, 4^{\prime \prime \prime}-H\right), 4.55\left(\mathrm{~m}, 1 \mathrm{H}, 3 \mathrm{a}{ }^{\prime \prime}-H\right), 4.98\left(\mathrm{~m}, 1 \mathrm{H}, 6^{\prime \prime \prime}-H\right)$, $5.21(\mathrm{~m}, 0.2 \mathrm{H}, 6 \mathrm{a}$ '"- $H$ minor), $5.25(\mathrm{~m}, 1.8 \mathrm{H}, 6 \mathrm{a}$ '”- $H$ major/OH), 7.02 (bs, $0.2 \mathrm{H}, 6^{\prime \prime}-\mathrm{H}$ minor), 7.07 (bs, $0.8 \mathrm{H}, 6^{\prime \prime}-\mathrm{H}$ major), $7.22-7.36$ (m, $2 \mathrm{H}, 2^{\prime \prime}-H / 5^{\prime \prime}-H$ ), 8.99 (d, $J=4.7 \mathrm{~Hz}, 0.2 \mathrm{H}, \mathrm{NH}$ minor), 9.38 (d, $J=4.0 \mathrm{~Hz}, 0.8 \mathrm{H}, \mathrm{NH}$ major). ${ }^{13} \mathrm{C}$ NMR (DMSO- $\left.d_{6}\right) \delta 13.1,15.0,22.4$, 24.1, 24.8, 27.0, 32.4, 34.1, 37.9, 61.9, 74.3, 82.2, 86.2, 112.0, 114.9, 117.1, 122.9, 123.2, 139.4, 146.9, 148.5, 148.8, 149.1, 150.4, 154.0, 169.4 .

4.49. 2-(((3aR,4S,6R,6aS)-6-(7-amino-5-(propylthio)-3H-[1,2,3] triazolo[4,5-d]pyrimidin-3-yl)-2,2-dimethyltetrahydro-3aHcyclopenta[d][1,3]dioxol-4-yl)oxy)ethanol (16c)

The solution of $14 \mathrm{~b} .9(0.5 \mathrm{~g}, 1.16 \mathrm{mmol})$ in THF $(10 \mathrm{~mL})$ was saturated with ammonia gas in a sealed vessel and left to react at room temperature for $1 \mathrm{~h}$. After evaporation of the solvent, the residue was purified by column chromatography on silica gel using hexane/ethyl acetate gradient to give $\mathbf{1 6 c}$ as a white solid $(0.36 \mathrm{~g}$, $75 \%$ yield). ${ }^{1} \mathrm{H}$ NMR (DMSO- $\left.d_{6}\right) \delta 1.00(\mathrm{t}, J=7.3 \mathrm{~Hz}, 3 \mathrm{H}$, $\left.\mathrm{SCH}_{2} \mathrm{CH}_{2} \mathrm{CH}_{3}\right), 1.27\left(\mathrm{~s}, 3 \mathrm{H}, \mathrm{CH}_{3}\right), 1.49\left(\mathrm{~s}, 3 \mathrm{H}, \mathrm{CH}_{3}\right), 1.71(\mathrm{~h}, J=7.2 \mathrm{~Hz}$, $\left.2 \mathrm{H}, \mathrm{SCH}_{2} \mathrm{CH}_{2} \mathrm{CH}_{3}\right), 2.56\left(\mathrm{dt}, J=13.0 \mathrm{~Hz} / 9.7 \mathrm{~Hz}, 1 \mathrm{H}, 5^{\prime}-\mathrm{Ha}\right), 2.67(\mathrm{dt}$, $\left.J=13.0 \mathrm{~Hz} / 6.6 \mathrm{~Hz}, 1 \mathrm{H}, 5^{\prime}-\mathrm{Hb}\right), 3.08\left(\mathrm{~m}, 2 \mathrm{H}, \mathrm{SCH}_{2} \mathrm{CH}_{2} \mathrm{CH}_{3}\right), 3.40-3.51$ $\left(\mathrm{m}, 4 \mathrm{H}, \mathrm{OCH}_{2} \mathrm{CH}_{2} \mathrm{OH}\right), 4.01\left(\mathrm{~m}, 1 \mathrm{H}, 4^{\prime}-H\right), 4.56(\mathrm{t}, J=5.2 \mathrm{~Hz}, 1 \mathrm{H}, \mathrm{OH})$, 4.67 (dd, $\left.J=7.3 \mathrm{~Hz} / 3.1 \mathrm{~Hz}, 1 \mathrm{H}, 3 \mathrm{a}^{\prime}-H\right), 5.02\left(\mathrm{~m}, 1 \mathrm{H}, 6^{\prime}-H\right), 5.22$ (dd, $J=7.2 \mathrm{~Hz} / 4.9 \mathrm{~Hz}, 1 \mathrm{H}, 6 \mathrm{a}^{\prime}-\mathrm{H}$ ), 8.10 (bs, $\left.1 \mathrm{H}, \mathrm{NHa}\right), 8.44$ (bs, $1 \mathrm{H}, \mathrm{NHb}$ ). ${ }^{13} \mathrm{C}$ NMR (DMSO- $\left.d_{6}\right) \delta 13.4,22.6,24.8,26.9,32.2,35.3,60.0,61.4$, $70.7,81.9,82.0,83.8,112.4,122.8,149.4,155.1,169.4$.

4.50. 2-(((3aR,4S,6R,6aS)-6-(7-(Cyclopropylamino)-5-(propylthio)$3 \mathrm{H}$-[1,2,3]triazolo[4,5-d]pyrimidin-3-yl)-2,2-dimethyltetrahydro3aH-cyclopenta[d][1,3]dioxol-4-yl)oxy)ethanol (16d)

The mixture of $\mathbf{1 4 b . 9}$ (0.5 g, $1.16 \mathrm{mmol})$, cyclopropylamine $(0.12 \mathrm{~g}, 1.74 \mathrm{mmol})$ and triethylamine $(0.21 \mathrm{~mL}, 1.45 \mathrm{mmol})$ in acetonitrile $(10 \mathrm{~mL})$ was heated at $80^{\circ} \mathrm{C}$ for $2 \mathrm{~h}$. After evaporation of the solvent, the residue was purified by column chromatography on silica gel using hexane/ethyl acetate gradient to give $\mathbf{1 6 d}$ as a white solid $\left(0.48 \mathrm{~g}, 91 \%\right.$ yield). ${ }^{1} \mathrm{H}$ NMR (DMSO- $\left.d_{6}\right) \delta 0.68\left(\mathrm{~m}, 2 \mathrm{H}, 2^{\prime}-\mathrm{Ha} / \mathrm{3}^{\prime}-\right.$ $\mathrm{Ha}), 0.76\left(\mathrm{~m}, 1.4 \mathrm{H}, 2^{\prime}-\mathrm{Hb}\right.$ major/3'-Hb major), 0.87 ( $\mathrm{m}, 0.6 \mathrm{H}, 2^{\prime}-\mathrm{Hb}$ minor $/ 3^{\prime}-\mathrm{Hb}$ minor), $1.00\left(\mathrm{t}, J=7.3 \mathrm{~Hz}, 3 \mathrm{H}, \mathrm{SCH}_{2} \mathrm{CH}_{2} \mathrm{CH}_{3}\right), 1.26(\mathrm{~s}, 3 \mathrm{H}$, $\left.\mathrm{CH}_{3}\right), 1.49\left(\mathrm{~s}, 3 \mathrm{H}, \mathrm{CH}_{3}\right), 1.73\left(\mathrm{~m}, 2 \mathrm{H}, \mathrm{SCH}_{2} \mathrm{CH}_{2} \mathrm{CH}_{3}\right), 2.54\left(\mathrm{~m}, 1 \mathrm{H}, 5^{\prime \prime}-\right.$ $\mathrm{Ha}), 2.65\left(\mathrm{~m}, 1 \mathrm{H}, 5^{\prime \prime}-\mathrm{Hb}\right), 3.05\left(\mathrm{~m}, 0.7 \mathrm{H}, 1^{\prime}-\mathrm{H}\right.$ major), $3.10(\mathrm{~m}, 2 \mathrm{H}$, $\left.\mathrm{SCH}_{2} \mathrm{CH}_{2} \mathrm{CH}_{3}\right), 3.39-3.51\left(\mathrm{~m}, 4.3 \mathrm{H}, 1^{\prime}-\mathrm{H}\right.$ minor $\left./ \mathrm{OCH}_{2} \mathrm{CH}_{2} \mathrm{OH}\right), 4.00$ $\left(\mathrm{m}, 1 \mathrm{H}, 4^{\prime \prime}-H\right), 4.57$ (bs, $\left.1 \mathrm{H}, \mathrm{OH}\right), 4.67$ (dd, $J=7.3 \mathrm{~Hz} / 3.2 \mathrm{~Hz}, 1 \mathrm{H}, 6 \mathrm{a}$ $H), 5.01\left(\mathrm{~m}, 1 \mathrm{H}, 6^{\prime \prime}-H\right), 5.20(\mathrm{~m}, 1 \mathrm{H}, 3 \mathrm{a} "-H), 8.83(\mathrm{~d}, J=4.1 \mathrm{~Hz}, 0.3 \mathrm{H}$, $\mathrm{NH}$ minor), 9.11 (d, $J=4.3 \mathrm{~Hz}, 0.7 \mathrm{H}, \mathrm{NH}$ major). ${ }^{13} \mathrm{C}$ NMR (DMSO- $d_{6}$ ) $\delta$ 5.9, 7.5, 13.6, 22.7, 23.7, 24.7, 26.9, 32.5, 35.4, 60.0, 61.3, 70.7, 81.8, $82.0,83.7,112.5,148.9,154.1,169.4$.

4.51. 2-(((3aR,4S,6R,6aS)-2,2-dimethyl-6-(7-(((1R,2S)-2phenylcyclopropyl)amino)-5-(propylthio)-3H-[1,2,3]triazolo[4,5-d] pyrimidin-3-yl)tetrahydro-3aH-cyclopenta[d][1,3]dioxol-4-yl)oxy) ethanol (16e)

The mixture of $\mathbf{1 4 b . 9}(0.5 \mathrm{~g}, 1.16 \mathrm{mmol}),(1 R, 2 S)-2-$ phenylcyclopropanamine hydrochloride $(0.3 \mathrm{~g}, 1.74 \mathrm{mmol})$ and triethylamine $(0.21 \mathrm{~mL}, 1.45 \mathrm{mmol})$ in acetonitrile $(10 \mathrm{~mL})$ was heated at $80^{\circ} \mathrm{C}$ for $2 \mathrm{~h}$. After evaporation of the solvent, the residue was purified by column chromatography on silica gel using hexane/ ethyl acetate gradient to give $16 e$ as a white solid ( $0.54 \mathrm{~g}, 88 \%$ yield). ${ }^{1} \mathrm{H}$ NMR (DMSO- $\left.d_{6}\right) \delta 0.83\left(\mathrm{t}, J=7.3 \mathrm{~Hz}, 2.4 \mathrm{H}, \mathrm{SCH}_{2} \mathrm{CH}_{2} \mathrm{CH}_{3}\right.$ major), $1.00\left(\mathrm{~m}, 0.6 \mathrm{H}, \mathrm{SCH}_{2} \mathrm{CH}_{2} \mathrm{CH}_{3}\right.$ minor), $1.27\left(\mathrm{~s}, 3 \mathrm{H}, \mathrm{CH}_{3}\right), 1.33\left(\mathrm{~m}, 1 \mathrm{H}, 3^{\prime}-\right.$ $\mathrm{Ha}), 1.40\left(\mathrm{~m}, 0.2 \mathrm{H}, 3^{\prime}-\mathrm{Hb}\right.$ minor), $1.50\left(\mathrm{~m}, 5.4 \mathrm{H}, \mathrm{CH}_{3} / 3^{\prime}-\mathrm{Hb}\right.$ major/ $\mathrm{SCH}_{2} \mathrm{CH}_{2} \mathrm{CH}_{3}$ major), 1.71 (h, $J=7.1 \mathrm{~Hz}, 0.4 \mathrm{H}, \mathrm{SCH}_{2} \mathrm{CH}_{2} \mathrm{CH}_{3}$ minor), $2.14\left(\mathrm{~m}, 0.8 \mathrm{H}, 2^{\prime}-\mathrm{H}\right.$ major), $2.26\left(\mathrm{~m}, 0.2 \mathrm{H}, 2^{\prime}-\mathrm{H}\right.$ minor $), 2.51(\mathrm{~m}, 1 \mathrm{H}$, $\left.5^{\prime \prime \prime}-\mathrm{Ha}\right), 2.66\left(\mathrm{~m}, 1 \mathrm{H}, 5^{\prime \prime \prime}-\mathrm{Hb}\right), 2.91\left(\mathrm{~m}, 1.6 \mathrm{H}, \mathrm{SCH}_{2} \mathrm{CH}_{2} \mathrm{CH}_{3}\right.$ major), $3.09\left(\mathrm{~m}, 0.4 \mathrm{H}, \mathrm{SCH}_{2} \mathrm{CH}_{2} \mathrm{CH}_{3}\right.$ minor), $3.20\left(\mathrm{~m}, 0.8 \mathrm{H}, 1^{\prime}-\mathrm{H}\right.$ major), 3.39-3.50 (m, $\left.4 \mathrm{H}, \mathrm{OCH}_{2} \mathrm{CH}_{2} \mathrm{OH}\right), 3.82\left(\mathrm{~m}, 0.2 \mathrm{H}, 1^{\prime}-\mathrm{H}\right.$ minor $), 4.00$ $\left(\mathrm{m}, 1 \mathrm{H}, 4^{\prime \prime \prime}-H\right), 4.57(\mathrm{t}, J=4.6 \mathrm{~Hz}, 1 \mathrm{H}, \mathrm{OH}), 4.67\left(\mathrm{~m}, \mathrm{z}, 1 \mathrm{H}, 6 \mathrm{a} \mathrm{a}^{\prime \prime}-\mathrm{H}\right)$, $5.01\left(\mathrm{~m}, 1 \mathrm{H}, 6^{\prime \prime \prime}-H\right), 5,20(\mathrm{~m}, 1 \mathrm{H}, 3 \mathrm{a} " '-H), 7.19\left(\mathrm{~m}, 3 \mathrm{H}, 2^{\prime \prime}-H / 4^{\prime \prime}-H / 6^{\prime \prime}-\right.$ $H), 7.29\left(\mathrm{t}, J=7.5 \mathrm{~Hz}, 2 \mathrm{H}, 3^{\prime \prime}-H / 5^{\prime \prime}-H\right), 8.99(\mathrm{~d}, J=4.4 \mathrm{~Hz}, 0.2 \mathrm{H}, \mathrm{NH}$ minor), 9.37 (d, $J=3.6 \mathrm{~Hz}, 0.8 \mathrm{H}, \mathrm{NH}$ major). ${ }^{13} \mathrm{C}$ NMR (DMSO- $d_{6}$ ) $\delta$ 13.1, 15.0, 22.4, 24.4, 24.8, 26.9, 32.5, 33.8, 35.4, 60.1, 61.4, 70.7, 81.9, 82.0, 83.7, 122.5, 125.7, 126.0, 128.2, 141.2, 149.1, 153.9, 169.5 .

4.52. 2-(((3aR,4S,6R,6aS)-6-(7-((3,4-difluorophenethyl)amino)-5(propylthio)-3H-[1,2,3]triazolo[4,5-d]pyrimidin-3-yl)-2,2dimethyltetrahydro-3aH-cyclopenta[d][1,3]dioxol-4-yl)oxy)ethanol (16f)

The mixture of 14b.9 (0.5 g, $1.16 \mathrm{mmol}), 2$-(3,4-difluorophenyl) ethanamine $(0.27 \mathrm{~g}, 1.74 \mathrm{mmol})$ and triethylamine $(0.21 \mathrm{~mL}$, $1.45 \mathrm{mmol})$ in acetonitrile $(10 \mathrm{~mL})$ was heated at $80^{\circ} \mathrm{C}$ for $2 \mathrm{~h}$. After evaporation of the solvent, the residue was purified by column chromatography on silica gel using hexane/ethyl acetate gradient to give $16 \mathbf{f}$ as a white solid ( $0.53 \mathrm{~g}, 83 \%$ yield). ${ }^{1} \mathrm{H}$ NMR (DMSO- $\left.d_{6}\right)$ $\delta 0.99\left(\mathrm{t}, J=7.1 \mathrm{~Hz}, 3 \mathrm{H}, \mathrm{SCH}_{2} \mathrm{CH}_{2} \mathrm{CH}_{3}\right), 1.26\left(\mathrm{~s}, 3 \mathrm{H}, \mathrm{CH}_{3}\right), 1.48(\mathrm{~s}, 3 \mathrm{H}$, $\left.\mathrm{CH}_{3}\right), 1.70\left(\mathrm{~h}, \mathrm{~J}=7.3 \mathrm{~Hz}, 2 \mathrm{H}, \mathrm{SCH}_{2} \mathrm{CH}_{2} \mathrm{CH}_{3}\right), 2.50\left(\mathrm{~m}, 1 \mathrm{H}, 5^{\prime \prime \prime}-\mathrm{Ha}\right), 2.65$ $\left(\mathrm{m}, 1 \mathrm{H}, 5^{\prime \prime \prime}-\mathrm{Hb}\right), 2.95\left(\mathrm{~m}, 2 \mathrm{H}, 2^{\prime}-\mathrm{H}_{2}\right), 3.07\left(\mathrm{~m}, 2 \mathrm{H}, \mathrm{SCH}_{2} \mathrm{CH}_{2} \mathrm{CH}_{3}\right)$, 3.40-3.51 (m, $\left.4 \mathrm{H}, \mathrm{OCH}_{2} \mathrm{CH}_{2} \mathrm{OH}\right), 3.73\left(\mathrm{q}, J=6.8 \mathrm{~Hz}, 1.6 \mathrm{H}, 1^{\prime}-\mathrm{H}_{2}\right.$ major), $4.00\left(\mathrm{~m}, 1 \mathrm{H}, 4^{\prime \prime \prime}-H\right), 4.10$ (q, $J=6.9 \mathrm{~Hz}, 0.4 \mathrm{H}, 1^{\prime}-\mathrm{H}_{2}$ minor), 4.58 (t, $J=5.0 \mathrm{~Hz}, 1 \mathrm{H}, \mathrm{OH}), 4.67$ (dd, $J=7.3 \mathrm{~Hz} / 3.2 \mathrm{~Hz}, 1 \mathrm{H}, 3 \mathrm{a} " '-H)$, $5.01\left(\mathrm{~m}, 1 \mathrm{H}, 6^{\prime \prime \prime}-H\right), 5.19\left(\mathrm{~m}, 0.2 \mathrm{H}, 6 \mathrm{a}{ }^{\prime \prime}-H\right), 7.07\left(\mathrm{~m}, 0.8 \mathrm{H}, 6^{\prime \prime}-\mathrm{H}\right.$ major), 7.15 ( $\mathrm{m}, 0.2 \mathrm{H}, 6^{\prime \prime}-\mathrm{H}$ minor), 7.29-7.41 (m, 2H, 2" $\left.-H / 5^{\prime \prime}-H\right), 8.74$ (t, $J=6.3 \mathrm{~Hz}, 0.2 \mathrm{H}, \mathrm{NH}$ minor $), 9.09$ (d, $J=5.6 \mathrm{~Hz}, 0.8 \mathrm{H}, \mathrm{NH}$ major). ${ }^{13} \mathrm{C}$ NMR (DMSO- $\left.d_{6}\right) \delta 13.3,22.7,24.7,26.9,32.4,33.5,35.4,41.1$, $60.0,61.3,70.7,81.8,83.7,112.5,117.0,117.6,123.0,125.4,137.1,147.0$, $148.2,149.0,149.1,150.4,153.0,169.4$.

4.53. 2-(((3aR,4S,6R,6aS)-6-(7-(((1R,2S)-2-(3,4-difluorophenyl) cyclopropyl)amino)-5-(propylthio)-3H-[1,2,3]triazolo[4,5-d] pyrimidin-3-yl)-2,2-dimethyltetrahydro-3aH-cyclopenta[d][1,3] dioxol-4-yl)oxy)ethanol (16g)

The mixture of $14 \mathrm{~b} .9$ (0.5 g, $1.16 \mathrm{mmol}),(1 R, 2 S)-2-(3,4-$ difluorophenyl)cyclopropanamine $(0.3 \mathrm{~g}, 1.74 \mathrm{mmol})$ and triethylamine $(0.21 \mathrm{~mL}, 1.45 \mathrm{mmol})$ in acetonitrile $(10 \mathrm{~mL})$ was heated at $80{ }^{\circ} \mathrm{C}$ for $2 \mathrm{~h}$. After evaporation of the solvent, the residue was purified by column chromatography on silica gel using hexane/ ethyl acetate gradient to give $\mathbf{1 6 g}$ as a white solid $(0.56 \mathrm{~g}, 86 \%$ yield). ${ }^{1} \mathrm{H}$ NMR (DMSO- $\left.d_{6}\right) \delta 0.84\left(\mathrm{t}, J=7.4 \mathrm{~Hz}, 2.4 \mathrm{H}, \mathrm{SCH}_{2} \mathrm{CH}_{2} \mathrm{CH}_{3}\right.$ major), $1.00\left(\mathrm{t}, J=7.3 \mathrm{~Hz}, 0.6 \mathrm{H}, \mathrm{SCH}_{2} \mathrm{CH}_{2} \mathrm{CH}_{3}\right.$ minor), $1.26\left(\mathrm{~s}, 3 \mathrm{H}, \mathrm{CH}_{3}\right), 1.38$ (m, $\left.1 \mathrm{H}, 3^{\prime}-\mathrm{Ha}\right), 1.48\left(\mathrm{~s}, 3 \mathrm{H}, \mathrm{CH}_{3}\right), 1.53\left(\mathrm{~m}, 2.6 \mathrm{H}, 3^{\prime}-\mathrm{Hb} / \mathrm{SCH}_{2} \mathrm{CH}_{2} \mathrm{CH}_{3}\right.$ major), $1.71\left(\mathrm{~h}, J=7.2 \mathrm{~Hz}, 0.4 \mathrm{H}, \mathrm{SCH}_{2} \mathrm{CH}_{2} \mathrm{CH}_{3}\right.$ minor), $2.13(\mathrm{~m}, 0.8 \mathrm{H}$, $2^{\prime}-H$ major), 2.24 (m, 0.2H, 2'-H minor), 2.50 ( $\left.\mathrm{m}, 1 \mathrm{H}, 5^{\prime \prime \prime}-\mathrm{Ha}\right), 2.65$ (m, 1H, 5"'-Hb), 2.89 (m, 0.8H, SCHa major), 2.93 (m, 0.8H, SCHb major), 3.08 ( $\mathrm{m}, 0.4 \mathrm{H}, \mathrm{SCH} 2$ minor), 3.15 ( $\mathrm{m}, 0.8 \mathrm{H}, 1^{\prime}-\mathrm{H}$ major), 3.39-3.51 ( $\left.\mathrm{m}, 4 \mathrm{H}, \mathrm{OCH}_{2} \mathrm{CH}_{2} \mathrm{OH}\right), 3.75\left(\mathrm{~m}, 0.2 \mathrm{H}, 1^{\prime}-\mathrm{H}\right.$ minor $), 4.01(\mathrm{~m}$, $\left.1 \mathrm{H}, 4^{\prime \prime \prime}-H\right), 4.56(\mathrm{t}, J=5.2 \mathrm{~Hz}, 1 \mathrm{H}, \mathrm{OH}), 4.64(\mathrm{dd}, J=7.3 \mathrm{~Hz} / 3.2 \mathrm{~Hz}$, $0.2 \mathrm{H}, 3 \mathrm{a}$ '"- $H$ minor), 4.68 (dd, $J=7.3 \mathrm{~Hz} / 3.2 \mathrm{~Hz}, 0.8 \mathrm{H}, 3 \mathrm{a}$ "'- $H$ major), 
5.01 (m, 1H, 6"' - H), 5.17 (m, 0.2H, 6a"'-H minor), 5.21 (m, 0.8H, 6a"'$H$ major), 7.02 (bs, $0.2 \mathrm{H}, 6^{\prime \prime}-H$ minor), 7.08 (bs, $0.8 \mathrm{H}, 6^{\prime \prime}-H$ major), $7.22-7.37$ ( $\mathrm{m}, 2 \mathrm{H}, 2^{\prime \prime}-H / 5^{\prime \prime}-H$ ), 9.00 (d, $J=4.7 \mathrm{~Hz}, 0.2 \mathrm{H}, \mathrm{NH}$ minor), $9.39\left(\mathrm{~d}, J=3.9 \mathrm{~Hz}, 0.8 \mathrm{H}, \mathrm{NH}\right.$ major). ${ }^{13} \mathrm{C}$ NMR (DMSO- $\left.d_{6}\right) \delta 13.1,15.0$, 22.4, 24.0, 24.8, 26.9, 32.4, 34.0, 35.5, 59.8, 60.0, 61.4, 70.7, 81.9, 83.7, $112.5,114.9,117.0,122.9,123.1,139.3,146.9,148.5,148.8,149.1,150.5$, $153.9,169.5$.

4.54. (3aR,4S,6R,6aS)-6-(7-amino-5-((2-hydroxypropyl)thio)-3H[1,2,3]triazolo[4,5-d]pyrimidin-3-yl)-2,2-dimethyltetrahydro-3aHcyclopenta[d][1,3]dioxol-4-ol (16h)

The mixture of 15a.1 ( $0.3 \mathrm{~g}, 0.92 \mathrm{mmol}), 1$-mercaptopropan-2-ol $(0.12 \mathrm{~mL}, 1.38 \mathrm{mmol})$ and $\mathrm{K}_{2} \mathrm{CO}_{3}(0.19 \mathrm{~g}, 1.38 \mathrm{mmol})$ in acetonitrile $(3 \mathrm{~mL})$ was heated in a sealed vessel at $100{ }^{\circ} \mathrm{C}$ for $1 \mathrm{~h}$. After evaporation of the solvent, the residue was taken up with water $(25 \mathrm{~mL})$ and extracted with dichloromethane $(3 \times 25 \mathrm{~mL})$. The combined organic layers were dried over $\mathrm{MgSO}_{4}$, evaporated and purified by column chromatography on silica gel using hexane/ethyl acetate gradient to give $\mathbf{1 6 h}$ as an oily residue $(0.27 \mathrm{~g}, 77 \%$ yield $) .{ }^{1} \mathrm{H}$ NMR $\left(\mathrm{DMSO}_{-} \mathrm{d}_{6}\right) \delta 1.18\left(\mathrm{~d}, J=6.2 \mathrm{~Hz}, 3 \mathrm{H}, \mathrm{CHOHCH}_{3}\right), 1.26\left(\mathrm{~s}, 3 \mathrm{H}, \mathrm{CH}_{3}\right), 1.47$ (s, 3H, $\left.\mathrm{CH}_{3}\right), 2.46\left(\mathrm{~m}, 1 \mathrm{H}, 5^{\prime}-\mathrm{Ha}\right), 2.57\left(\mathrm{~m}, 1 \mathrm{H}, 5^{\prime}-\mathrm{Hb}\right), 3.10$ (ddd, $J=13.4 \mathrm{~Hz} / 6.6 \mathrm{~Hz} / 4.3 \mathrm{~Hz}, 1 \mathrm{H}, \mathrm{SCHa}), 3.22(\mathrm{dt}, J=13.3 \mathrm{~Hz} / 5.4 \mathrm{~Hz}, 1 \mathrm{H}$, $\mathrm{SCHb}$ ), 3.89 (ddt, $\left.J=11.6 \mathrm{~Hz} / 8.6 \mathrm{~Hz} / 4.1 \mathrm{~Hz}, 1 \mathrm{H}, \mathrm{CHOHCH}_{3}\right), 4.13(\mathrm{~m}$, $\left.1 \mathrm{H}, 4^{\prime}-H\right), 4.55\left(\mathrm{~m}, 1 \mathrm{H}, 3 \mathrm{a}^{\prime}-H\right), 4.86\left(\mathrm{dd}, J=4.9 \mathrm{~Hz} / 1.4 \mathrm{~Hz}, 1 \mathrm{H}, 4^{\prime}-\mathrm{OH}\right)$, 4.98 (ddd, $\left.J=9.0 \mathrm{~Hz} / 7.0 \mathrm{~Hz} / 4.3 \mathrm{~Hz}, 1 \mathrm{H}, 6^{\prime}-H\right), 5.25\left(\mathrm{~m}, 1 \mathrm{H}, 6 \mathrm{a}^{\prime}-H\right)$, 5.27 (d, $J=4.8 \mathrm{~Hz}, 1 \mathrm{H}, \mathrm{CHOHCH}_{3}$ ), 8.10 (bs, 1H, NHa), 8.43 (bs, $1 \mathrm{H}$, $\mathrm{NHb}$ ). ${ }^{13} \mathrm{C}$ NMR (DMSO-d $\left.\mathrm{d}_{6}\right) \delta 22.7,24.8,26.9,37.8,47.8,61.8,65.4$, 74.2, 82.1, 86.1, 111.9, 122.8, 149.3, 155.1, 169.5.

4.55. (3aR,4S,6R,6aS)-6-(7-amino-5-((3-hydroxypropyl)thio)-3H[1,2,3]triazolo[4,5-d]pyrimidin-3-yl)-2,2-dimethyltetrahydro-3aHcyclopenta[d][1,3]dioxol-4-ol (16i)

The mixture of 15a.1 (0.3 g, $0.92 \mathrm{mmol}$ ), 3-mercapto-1-propanol $(0.12 \mathrm{~mL}, 1.38 \mathrm{mmol})$ and $\mathrm{K}_{2} \mathrm{CO}_{3}(0.19 \mathrm{~g}, 1.38 \mathrm{mmol})$ in acetonitrile $(3 \mathrm{~mL})$ was heated in a sealed vessel at $100{ }^{\circ} \mathrm{C}$ for $1 \mathrm{~h}$. After evaporation of the solvent, the residue was taken up with water $(25 \mathrm{~mL})$ and extracted with dichloromethane $(3 \times 25 \mathrm{~mL})$. The combined organic layers were dried over $\mathrm{MgSO}_{4}$, evaporated and purified by column chromatography on silica gel using hexane/ethyl acetate gradient to give $16 \mathbf{i}$ as an oily residue $(0.25 \mathrm{~g}, 71 \%$ yield $) .{ }^{1} \mathrm{H}$ NMR $\left(\mathrm{DMSO}_{-} \mathrm{d}_{6}\right) \delta 1.26\left(\mathrm{~s}, 3 \mathrm{H}, \mathrm{CH}_{3}\right), 1.47\left(\mathrm{~s}, 3 \mathrm{H}, \mathrm{CH}_{3}\right), 1.84(\mathrm{~m}, 2 \mathrm{H}$, $\left.\mathrm{SCH}_{2} \mathrm{CH}_{2} \mathrm{CH}_{2} \mathrm{OH}\right), 2.48\left(\mathrm{~m}, 1 \mathrm{H}, 5^{\prime}-\mathrm{Ha}\right), 2.55\left(\mathrm{~m}, 1 \mathrm{H}, 5^{\prime}-\mathrm{Hb}\right), 3.14(\mathrm{t}$, $J=7.2 \mathrm{~Hz}, 2 \mathrm{H}, \mathrm{SCH} 2), 3.52\left(\mathrm{t}, J=6.2 \mathrm{~Hz}, 1 \mathrm{H}, \mathrm{CH}_{2} \mathrm{OH}\right), 4.13\left(\mathrm{~m}, 1 \mathrm{H}, 4^{\prime}-\right.$ $H), 4.55\left(\mathrm{~m}, 2 \mathrm{H}, 3 \mathrm{a}^{\prime}-\mathrm{H} / \mathrm{CH}_{2} \mathrm{OH}\right), 4.98$ (ddd, $J=9.1 \mathrm{~Hz} / 7.0 \mathrm{~Hz} / 4.4 \mathrm{~Hz}$, $\left.1 \mathrm{H}, 6^{\prime}-H\right), 5.26\left(\mathrm{~m}, 2 \mathrm{H}, 6 \mathrm{a}^{\prime}-\mathrm{H} / 4^{\prime}-\mathrm{OH}\right), 8.09$ (bs, $\left.1 \mathrm{H}, \mathrm{NHa}\right), 8.43$ (bs, $1 \mathrm{H}$, NHb). ${ }^{13} \mathrm{C}$ NMR (DMSO- $\left.d_{6}\right) \delta 24.7,26.9,27.2,32.3,37.7,59.5,61.9$, $74.2,82.0,86.1,111.9,122.8,149.4,155.1,169.3$.

4.56. (3aR,4S,6R,6aS)-6-(7-(((1R,2S)-2-(3,4-difluorophenyl) cyclopropyl)amino)-5-((3-hydroxypropyl)thio)-3H-[1,2,3]triazolo [4,5-d]pyrimidin-3-yl)-2,2-dimethyltetrahydro-3aH-cyclopenta[d] [1,3]dioxol-4-ol (16j)

The mixture of 15a.2 ( $0.3 \mathrm{~g}, 0.63 \mathrm{mmol})$, 3-mercapto-1-propanol $(0.08 \mathrm{~mL}, 0.96 \mathrm{mmol})$ and $\mathrm{K}_{2} \mathrm{CO}_{3}(0.13 \mathrm{~g}, 0.96 \mathrm{mmol})$ in acetonitrile $(3 \mathrm{~mL})$ was heated in a sealed vessel at $100{ }^{\circ} \mathrm{C}$ for $1 \mathrm{~h}$. After evaporation of the solvent, the residue was taken up with water $(25 \mathrm{~mL})$ and extracted with dichloromethane $(3 \times 25 \mathrm{~mL})$. The combined organic layers were dried over $\mathrm{MgSO}_{4}$, evaporated and purified by column chromatography on silica gel using hexane/ethyl acetate gradient to give $\mathbf{1 6} \mathbf{j}$ as a white solid $(0.24 \mathrm{~g}, 72 \%$ yield $) .{ }^{1} \mathrm{H}$ NMR (DMSO-d $\left.d_{6}\right) \delta 1.26\left(\mathrm{~s}, 3 \mathrm{H}, \mathrm{CH}_{3}\right), 1.38\left(\mathrm{q}, J=6.3 \mathrm{~Hz}, 1 \mathrm{H}, 3^{\prime}-\mathrm{Ha}\right.$ ), 1.47 (s, $\left.3 \mathrm{H}, \mathrm{CH}_{3}\right), 1.52$ (dt, $\left.J=9.9 \mathrm{~Hz} / 5.4 \mathrm{~Hz}, 1 \mathrm{H}, 3^{\prime}-\mathrm{Hb}\right), 1.70$ (p, $J=6.6 \mathrm{~Hz}$,
1.6H, $\mathrm{SCH}_{2} \mathrm{CH}_{2} \mathrm{CH}_{2} \mathrm{OH}$ major), 1.84 (p, $J=6.7 \mathrm{~Hz}, 0.4 \mathrm{H}$, $\mathrm{SCH}_{2} \mathrm{CH}_{2} \mathrm{CH}_{2} \mathrm{OH}$ minor), $2.14\left(\mathrm{~m}, 0.8 \mathrm{H}, 2^{\prime}-\mathrm{H}\right.$ major), $2.25(\mathrm{~m}, 0.2 \mathrm{H}$, $2^{\prime}-\mathrm{H}$ minor), $2.44\left(\mathrm{~m}, 1 \mathrm{H}, 5^{\prime \prime \prime}-\mathrm{Ha}\right), 2.54\left(\mathrm{~m}, 1 \mathrm{H}, 5^{\prime \prime \prime}-\mathrm{Hb}\right), 2.97(\mathrm{dt}$, $J=13.8 \mathrm{~Hz} / 7.1 \mathrm{~Hz}, 0.8 \mathrm{H}$, SCHa major), 3.03 (dt, $J=13.9 \mathrm{~Hz} / 7.0 \mathrm{~Hz}$, $0.8 \mathrm{H}, \mathrm{SCHb}$ major), $3.16\left(\mathrm{~m}, 1.2 \mathrm{H}, \mathrm{SCH} 2 \mathrm{minor} / 1^{\prime}-H\right.$ major), 3.40 (q, $J=6.0 \mathrm{~Hz}, 1.6 \mathrm{H}, \mathrm{CH}_{2} \mathrm{OH}$ major), $3.53\left(\mathrm{q}, J=5.6 \mathrm{~Hz}, 0.4 \mathrm{H}, \mathrm{CH}_{2} \mathrm{OH}\right.$ minor), $3.76\left(\mathrm{~m}, 0.2 \mathrm{H}, 1^{\prime}-\mathrm{H}\right.$ minor), $4.13\left(\mathrm{~m}, 1 \mathrm{H}, 4^{\prime \prime \prime}-\mathrm{H}\right), 4.49(\mathrm{t}$, $J=5.1 \mathrm{~Hz}, 0.8 \mathrm{H}, \mathrm{CH}_{2} \mathrm{OH}$ major $), 4.55\left(\mathrm{~m}, 1.2 \mathrm{H}, \mathrm{CH}_{2} \mathrm{OH}\right.$ minor/3a"'- $\left.\mathrm{H}\right)$, $4.98\left(\mathrm{~m}, 1 \mathrm{H}, 6^{\prime \prime \prime}-H\right), 5.22(\mathrm{~m}, 0.2 \mathrm{H}, 6 \mathrm{a}$ "'-H minor), 5.26 (m, 1.8H, 6a"'$H$ major/4"' $-\mathrm{OH}$ ), 7.02 (bs, $0.2 \mathrm{H}, 6^{\prime \prime}-\mathrm{H}$ minor), 7.08 (bs, $0.8 \mathrm{H}, 6^{\prime \prime}-\mathrm{H}$ major), 7.22-7.36 (m, $\left.2 \mathrm{H}, 2^{\prime \prime}-H / 5^{\prime \prime}-H\right), 8.99$ (d, $J=4.6 \mathrm{~Hz}, 0.2 \mathrm{H}, \mathrm{NH}$ minor), 9.37 (d, $J=3.9 \mathrm{~Hz}, 0.8 \mathrm{H}, \mathrm{NH}$ major). ${ }^{13} \mathrm{C}$ NMR (DMSO- $d_{6}$ ) $\delta$ 15.0, 23.9, 24.7, 26.9, 27.3, 32.2, 33.9, 37.8, 59.3, 61.9, 74.3, 82.1, $86.1,111.9,115.0,117.0,122.9,123.2,139.2,146.9,148.4,149.0,150.4$, 154.0, 169.4 .

4.57. (3aR,4S,6R,6aS)-6-(7-(((1R,2S)-2-(3,4-difluorophenyl) cyclopropyl)amino)-5-(((R)-2-hydroxypropyl)thio)-3H-[1,2,3] triazolo[4,5-d]pyrimidin-3-yl)-2,2-dimethyltetrahydro-3aHcyclopenta[d][1,3]dioxol-4-ol (16k)

The mixture of 15a.2 (0.3 g, $0.63 \mathrm{mmol})$, (R)-1-mercaptopropan2-ol $(0.08 \mathrm{~mL}, 0.96 \mathrm{mmol})$ and $\mathrm{K}_{2} \mathrm{CO}_{3}(0.13 \mathrm{~g}, 0.96 \mathrm{mmol})$ in acetonitrile $(3 \mathrm{~mL})$ was heated in a sealed vessel at $100{ }^{\circ} \mathrm{C}$ for $1 \mathrm{~h}$. After evaporation of the solvent, the residue was taken up with water $(25 \mathrm{~mL})$ and extracted with dichloromethane $(3 \times 25 \mathrm{~mL})$. The combined organic layers were dried over $\mathrm{MgSO}_{4}$, evaporated and purified by column chromatography on silica gel using hexane/ ethyl acetate gradient to give $\mathbf{1 6 k}$ as a white solid $(0.25 \mathrm{~g}, 75 \%$ yield). ${ }^{1} \mathrm{H}$ NMR (DMSO- $\left.d_{6}\right) \delta 1.01\left(\mathrm{~d}, J=6.1 \mathrm{~Hz}, 2.4 \mathrm{H}, \mathrm{CHOHCH}_{3}\right.$ major), 1.18 (d, $J=6.0 \mathrm{~Hz}, 0.6 \mathrm{H}, \mathrm{CHOHCH}_{3}$ minor), $1.26\left(\mathrm{~s}, 3 \mathrm{H}, \mathrm{CH}_{3}\right.$ ), $1.38\left(\mathrm{~m}, 1 \mathrm{H}, 3^{\prime}-\mathrm{Ha}\right), 1.47\left(\mathrm{~s}, 3 \mathrm{H}, \mathrm{CH}_{3}\right), 1.53\left(\mathrm{~m}, 1 \mathrm{H}, 3^{\prime}-\mathrm{Hb}\right), 2.13(\mathrm{~m}$, $0.8 \mathrm{H}, 2^{\prime}-H$ major), 2.24 ( $\mathrm{m}, 0.2 \mathrm{H}, 2^{\prime}-\mathrm{H}$ minor), 2.43 ( $\mathrm{m}, 1 \mathrm{H}, 5^{\prime \prime \prime}-\mathrm{Ha}$ ), $2.55\left(\mathrm{~m}, 1 \mathrm{H}, 5^{\prime \prime \prime}-\mathrm{Hb}\right), 2.88$ (dd, $J=13.4 \mathrm{~Hz} / 7.0 \mathrm{~Hz}, 0.8 \mathrm{H}, \mathrm{SCHa}$ major), 3.10-3.18 (m, 1.8H, SCHa minor/SCHb major/1'-H major), 3.23 (dd, $J=13.3 \mathrm{~Hz} / 5.2 \mathrm{~Hz}, 0.2 \mathrm{H}, \mathrm{SCHb}$ minor $), 3.78\left(\mathrm{~m}, 1 \mathrm{H}, \mathrm{CHOHCH}_{3}\right.$ major/ $1^{\prime}-\mathrm{H}$ minor), 3.90 (m, $0.2 \mathrm{H}, \mathrm{CHOHCH}_{3}$ minor), 4.14 (bs, $1 \mathrm{H}, 4^{\prime \prime \prime}-\mathrm{H}$ ), $4.56\left(\mathrm{~m}, 1 \mathrm{H}, 3 \mathrm{a}\right.$ "'- $H$ ), 4.79 (d, $J=4.8 \mathrm{~Hz}, 0.8 \mathrm{H}, \mathrm{CHOHCH}_{3}$ major), 4.87 (d, $J=4.2 \mathrm{~Hz}, 0.2 \mathrm{H}, \mathrm{CHOHCH}_{3}$ minor), $4.99\left(\mathrm{~m}, 1 \mathrm{H}, 6^{\prime \prime \prime}-\mathrm{H}\right), 5.22(\mathrm{~m}$, $0.2 \mathrm{H}, 6 \mathrm{a}$ "'- $\mathrm{H}$ minor), $5.26(\mathrm{~m}, 1.8 \mathrm{H}, 6 \mathrm{a}$ "'- $\mathrm{H}$ major/4"'-OH), 7.02 (bs, $0.2 \mathrm{H}, 6^{\prime \prime}-\mathrm{H}$ minor), 7.09 (bs, 0.8H, 6" $-H$ major), 7.22-7.36 ( $\mathrm{m}, 2 \mathrm{H}, 2^{\prime \prime}-$ $\left.H / 5^{\prime \prime}-H\right), 9.00$ (d, $J=4.8 \mathrm{~Hz}, 0.2 \mathrm{H}, N \mathrm{H}$ minor), 9.37 (d, $J=4.0 \mathrm{~Hz}$, $0.8 \mathrm{H}, \mathrm{NH}$ major). ${ }^{13} \mathrm{C}$ NMR (DMSO- $\left.d_{6}\right) \delta 15.0,22.5,24.0,24.7,26.9$, 33.9, 37.8, 39.3, 61.8, 65.1, 74.3, 82.1, 86.1, 111.9, 114.9, 117.1, 123.0, $123.1,139.2,144.5,146.8,148.5,149.0,150.3,153.9,155.4,169.6$.

4.58. (3aR,4S,6R,6aS)-6-(7-(((1R,2S)-2-(3,4-difluorophenyl) cyclopropyl)amino)-5-(((S)-2-hydroxypropyl)thio)-3H-[1,2,3] triazolo[4,5-d]pyrimidin-3-yl)-2,2-dimethyltetrahydro-3aHcyclopenta[d][1,3]dioxol-4-ol (16l)

The mixture of 15a.2 (0.3 g, $0.63 \mathrm{mmol})$, (S)-1-mercaptopropan2-ol $(0.08 \mathrm{~mL}, 0.96 \mathrm{mmol})$ and $\mathrm{K}_{2} \mathrm{CO}_{3}(0.13 \mathrm{~g}, 0.96 \mathrm{mmol})$ in acetonitrile $(3 \mathrm{~mL})$ was heated in a sealed vessel at $100{ }^{\circ} \mathrm{C}$ for $1 \mathrm{~h}$. After evaporation of the solvent, the residue was taken up with water $(25 \mathrm{~mL})$ and extracted with dichloromethane $(3 \times 25 \mathrm{~mL})$. The combined organic layers were dried over $\mathrm{MgSO}_{4}$, evaporated and purified by column chromatography on silica gel using hexane/ ethyl acetate gradient to give $\mathbf{1 6 1}$ as a white solid ( $0.28 \mathrm{~g}, 84 \%$ yield). ${ }^{1} \mathrm{H}$ NMR (DMSO- $d_{6}$ ) $\delta 1.01$ (d, $J=6.1 \mathrm{~Hz}, 2.4 \mathrm{H}, \mathrm{CHOHCH}_{3}$ major), 1.18 (d, $J=6.0 \mathrm{~Hz}, 0.6 \mathrm{H}, \mathrm{CHOHCH}_{3}$ minor), $1.25\left(\mathrm{~s}, 3 \mathrm{H}, \mathrm{CH}_{3}\right), 1.37(\mathrm{~m}$, $0.8 \mathrm{H}, 3^{\prime}-\mathrm{Ha}$ major), 1.40 ( $\mathrm{m}, 0.2 \mathrm{H}, 3^{\prime}-\mathrm{Ha}$ minor), 1.47 (s, 3.2H, $3^{\prime}-\mathrm{Hb}$ minor $\left./ \mathrm{CH}_{3}\right), 1.51$ ( $\mathrm{m}, 0.8 \mathrm{H}, 3^{\prime}-\mathrm{Hb}$ major), 2.14 ( $\mathrm{m}, 0.8 \mathrm{H}, 2^{\prime}-\mathrm{H}$ major), $2.24\left(\mathrm{~m}, 0.2 \mathrm{H}, 2^{\prime}-\mathrm{H}\right.$ minor), $2.43\left(\mathrm{~m}, 1 \mathrm{H}, 4^{\prime \prime \prime}-\mathrm{Ha}\right), 2.55\left(\mathrm{~m}, 1 \mathrm{H}, 4^{\prime \prime \prime}-\right.$ $\mathrm{Hb}$ ), 3.06 ( $\mathrm{m}, 1.6 \mathrm{H}, \mathrm{SCH} 2$ major), 3.12 ( $\mathrm{m}, 0.2 \mathrm{H}, \mathrm{SCHa}$ minor), 3.17 
(m, 0.8H, $1^{\prime}-\mathrm{H}$ major), 3.23 (dd, $J=13.5 \mathrm{~Hz} / 5.1 \mathrm{~Hz}, 0.2 \mathrm{H}, \mathrm{SCH} b \mathrm{mi}-$ nor), $3.77\left(\mathrm{~m}, 1 \mathrm{H}, \mathrm{CHOHCH}_{3}\right.$ major $/ 1^{\prime}-\mathrm{H}$ minor $), 3.88(\mathrm{~m}, 0.2 \mathrm{H}$, $\mathrm{CHOHCH}_{3}$ minor), 4.13 (bs, $\left.1 \mathrm{H}, 4^{\prime \prime \prime}-H\right), 4.56(\mathrm{~m}, 1 \mathrm{H}, 3 \mathrm{a}$ '"- $-\mathrm{H}), 4.79$ (d, $J=4.8 \mathrm{~Hz}, 0.8 \mathrm{H}, \mathrm{CHOHCH}_{3}$ major $), 4.86(\mathrm{~d}, J=4.8 \mathrm{~Hz}, 0.2 \mathrm{H}$, $\mathrm{CHOHCH}_{3}$ minor), 4.99 (m, $\left.1 \mathrm{H}, 6^{\prime \prime \prime}-H\right), 5.20$ (m, 0.2H, 6a"'- $H$ minor), $5.25\left(\mathrm{~m}, 1.8 \mathrm{H}, 6 \mathrm{a}\right.$ "'- $\mathrm{H}$ major $\left./ 4^{\prime \prime \prime}-\mathrm{OH}\right), 7.02\left(\mathrm{~m}, 0.2 \mathrm{H}, 6^{\prime \prime}-\mathrm{H}\right.$ minor $), 7.08$

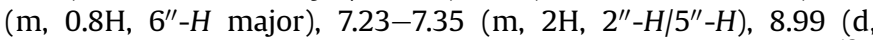
$J=4.2 \mathrm{~Hz}, 0.2 \mathrm{H}, \mathrm{NH}$ minor $), 9.36$ (d, $J=3.1 \mathrm{~Hz}, 0.8 \mathrm{H}, \mathrm{NH}$ major $).{ }^{13} \mathrm{C}$ NMR (DMSO- $\left.d_{6}\right) \delta 15.0,22.6,23.9,24.7,26.9,33.8,37.8,39.2,61.8$, $65.2,74.3,82.1,86.1,111.9,115.0,117.0,123.0,123.1,139.1,147.2$, $148.5,148.6,148.9,150.1,150.3,153.8,155.4,169.6$.

4.59. 1-((7-(((1R,2S)-2-(3,4-Difluorophenyl)cyclopropyl)amino)-3((3aS,4R,6S,6aR)-6-(2-hydroxyethoxy)-2,2-dimethyltetrahydro$3 a H$-cyclopenta[d][1,3]dioxol-4-yl)-3H-[1,2,3]triazolo[4,5-d] pyrimidin-5-yl)thio)propan-2-ol (16m)

The mixture of 15a.3 ( $0.3 \mathrm{~g}, 0.57 \mathrm{mmol}), 1$-mercaptopropan-2-ol $(0.07 \mathrm{~mL}, 0.87 \mathrm{mmol})$ and $\mathrm{K}_{2} \mathrm{CO}_{3}(0.12 \mathrm{~g}, 0.87 \mathrm{mmol})$ in acetonitrile $(3 \mathrm{~mL})$ was heated in a sealed vessel at $100{ }^{\circ} \mathrm{C}$ for $3 \mathrm{~h}$. After evaporation of the solvent, the residue was taken up with water $(25 \mathrm{~mL})$ and extracted with dichloromethane $(3 \times 25 \mathrm{~mL})$. The combined organic layers were dried over $\mathrm{MgSO}_{4}$, evaporated and purified by column chromatography on silica gel using hexane/ethyl acetate gradient to give $16 \mathrm{~m}$ as a white solid $(0.25 \mathrm{~g}, 74 \%$ yield $) .{ }^{1} \mathrm{H}$ NMR (DMSO- $\left.d_{6}\right) \delta 1.01\left(\mathrm{~d}, J=6.1 \mathrm{~Hz}, 1.2 \mathrm{H}, \mathrm{CHOHCH}_{3}\right.$ major $R$ or $\left.S\right), 1.05(\mathrm{~d}$, $J=6.1 \mathrm{~Hz}, 1.2 \mathrm{H}, \mathrm{CHOHCH}_{3}$ major $R$ or $\left.S\right), 1.18\left(\mathrm{~m}, 0.6 \mathrm{H}, \mathrm{CHOHCH}_{3}\right.$ minor), $1.27\left(\mathrm{~s}, 3 \mathrm{H}, \mathrm{CH}_{3}\right), 1.38\left(\mathrm{~m}, 1 \mathrm{H}, 3^{\prime}-\mathrm{Ha}\right), 1.49\left(\mathrm{~s}, 3 \mathrm{H}, \mathrm{CH}_{3}\right), 1.52$ $\left(\mathrm{m}, 1 \mathrm{H}, 3^{\prime}-H b\right), 2.14\left(\mathrm{~m}, 1 \mathrm{H}, 2^{\prime}-H\right.$ major $), 2.25\left(\mathrm{~m}, 1 \mathrm{H}, 2^{\prime}-\mathrm{H}\right.$ minor), $2.50\left(\mathrm{~m}, 1 \mathrm{H}, 5^{\prime \prime \prime}-\mathrm{Ha}\right), 2.66\left(\mathrm{~m}, 1 \mathrm{H}, 5^{\prime \prime \prime}-\mathrm{Hb}\right), 2.88(\mathrm{dd}, J=13.5 \mathrm{~Hz} /$ $7.0 \mathrm{~Hz}, 0.4 \mathrm{H}, \mathrm{SCHa}$ major $R$ or $S), 3.02(\mathrm{dd}, J=13.4 \mathrm{~Hz} / 6.8 \mathrm{~Hz}, 0.4 \mathrm{H}$, $\mathrm{SCHa}$ major $\mathrm{R}$ or $\mathrm{S}), 3.08-3.17$ (m, $2 \mathrm{H}, \mathrm{SCHa}$ minor $/ \mathrm{SCHb} / 1^{\prime}-\mathrm{H}$ major $), 3.40-3.50\left(\mathrm{~m}, 4 \mathrm{H}, \mathrm{OCH}_{2} \mathrm{CH}_{2} \mathrm{OH}\right), 3.76\left(\mathrm{~m}, 1 \mathrm{H}, \mathrm{CHOH}\right.$ major $/ 1^{\prime}-\mathrm{H}$ minor), $3.88(\mathrm{~m}, 0.2 \mathrm{H}, \mathrm{CHOH}$ minor $), 4.00\left(\mathrm{~m}, 1 \mathrm{H}, 4^{\prime \prime \prime}-\mathrm{H}\right), 4.56(\mathrm{t}$, $\left.J=4.5 \mathrm{~Hz}, 1 \mathrm{H}, \mathrm{OCH}_{2} \mathrm{CH}_{2} \mathrm{OH}\right), 4.67\left(\mathrm{~m}, 1 \mathrm{H}, 3 \mathrm{a} \mathrm{a}^{\prime}-\mathrm{H}\right), 4.79(\mathrm{~m}, 0.8 \mathrm{H}$, CHOH major), 4.85 (m, 0.2H, CHOH minor), $5.02\left(\mathrm{~m}, 1 \mathrm{H}, 6^{\prime \prime \prime}-\mathrm{H}\right), 5.17$ (m, 0.2H, 6a"'-H minor), 5.22 (m, 0.8H, 6a"'- $H$ major), 7.02 (bs, $0.2 \mathrm{H}$, $6^{\prime \prime}-H$ minor), 7.09 (bs, $1 \mathrm{H}, 6^{\prime \prime}-H$ major), $7.22-7.36$ (m, 2H, $2^{\prime \prime}-H / 5^{\prime \prime}-$ $H$ ), 9.00 (d, $J=4.5 \mathrm{~Hz}, 0.2 \mathrm{H}, \mathrm{NH}$ minor), 9.37 ( $\mathrm{m}, 0.8 \mathrm{H}, \mathrm{NH}$ major). ${ }^{13} \mathrm{C}$ NMR (DMSO- $\left.d_{6}\right) \delta 15.0,22.5,22.6,23.9,24.8,26.9,33.8,35.5$, 39.2, 60.0, 61.4, 65.1, 70.7, 81.9, 82.0, 83.7, 112.5, 115.0, 117.1, 123.0, $123.1,139.2,146.9,148.5,148.7,149.0,150.4,153.9,169.7$.

4.60. 3-((7-(((1R,2S)-2-(3,4-Difluorophenyl)cyclopropyl)amino)-3((3aS,4R,6S,6aR)-6-(2-hydroxyethoxy)-2,2-dimethyltetrahydro$3 a H$-cyclopenta[d][1,3]dioxol-4-yl)-3H-[1,2,3]triazolo[4,5-d] pyrimidin-5-yl)thio)propan-1-ol (16n)

The mixture of 15a.3 ( $0.3 \mathrm{~g}, 0.57 \mathrm{mmol})$, 3-mercapto-1-propanol $(0.07 \mathrm{~mL}, 0.87 \mathrm{mmol})$ and $\mathrm{K}_{2} \mathrm{CO}_{3}(0.12 \mathrm{~g}, 0.87 \mathrm{mmol})$ in acetonitrile $(3 \mathrm{~mL})$ was heated in a sealed vessel at $100{ }^{\circ} \mathrm{C}$ for $1 \mathrm{~h}$. After evaporation of the solvent, the residue was taken up with water $(25 \mathrm{~mL})$ and extracted with dichloromethane $(3 \times 25 \mathrm{~mL})$. The combined organic layers were dried over $\mathrm{MgSO}_{4}$, evaporated and purified by column chromatography on silica gel using hexane/ethyl acetate gradient to give $16 \mathrm{n}$ as a white solid $(0.22 \mathrm{~g}, 65 \%$ yield $) .{ }^{1} \mathrm{H}$ NMR $\left(\right.$ DMSO- $\left.d_{6}\right) \delta 1.27\left(\mathrm{~s}, 3 \mathrm{H}, \mathrm{CH}_{3}\right), 1.37\left(\mathrm{q}, J=6.2 \mathrm{~Hz}, 1 \mathrm{H}, 3^{\prime}-\mathrm{Ha}\right), 1.49(\mathrm{~s}$, $\left.3 \mathrm{H}, \mathrm{CH}_{3}\right), 1.52\left(\mathrm{dt}, J=10.4 \mathrm{~Hz} / 5.5 \mathrm{~Hz}, 1 \mathrm{H}, 3^{\prime}-\mathrm{Hb}\right), 1.70$ (p, $J=6.7 \mathrm{~Hz}$, $1.6 \mathrm{H}, \quad \mathrm{SCH}_{2} \mathrm{CH}_{2} \mathrm{CH}_{2} \mathrm{OH}$ major), $1.84(\mathrm{p}, J=6.7 \mathrm{~Hz}, 0.4 \mathrm{H}$, $\mathrm{SCH}_{2} \mathrm{CH}_{2} \mathrm{CH}_{2} \mathrm{OH}$ minor), 2.15 (ddd, $J=9.5 \mathrm{~Hz} / 6.4 \mathrm{~Hz} / 3.3 \mathrm{~Hz}, 0.8 \mathrm{H}$, $2^{\prime}-\mathrm{H}$ major), 2.24 (m, 0.2H, 2'-H minor), $2.51\left(\mathrm{~m}, 1 \mathrm{H}, 5^{\prime \prime \prime}-\mathrm{Ha}\right), 2.66$ $\left(\mathrm{m}, 1 \mathrm{H}, 5^{\prime \prime \prime}-\mathrm{Hb}\right), 3.00\left(\mathrm{~m}, 1.6 \mathrm{H}, \mathrm{SCH}_{2} \mathrm{CH}_{2} \mathrm{CH}_{2} \mathrm{OH}\right.$ major), $3.16(\mathrm{~m}, 1.2 \mathrm{H}$, $\mathrm{SCH}_{2} \mathrm{CH}_{2} \mathrm{CH}_{2} \mathrm{OH}$ minor $/ 1^{\prime}-\mathrm{H}$ major), 3.38-3.54 (m, $6 \mathrm{H}$, $\left.\mathrm{SCH}_{2} \mathrm{CH}_{2} \mathrm{CH}_{2} \mathrm{OH} / \mathrm{OCH}_{2} \mathrm{CH}_{2} \mathrm{OH}\right), 3.74\left(\mathrm{~m}, 0.2 \mathrm{H}, 1^{\prime}-\mathrm{H}\right.$ minor), 4.00 (m, $\left.1 \mathrm{H}, 4^{\prime \prime \prime}-H\right), 4.50\left(\mathrm{t}, J=5.1 \mathrm{~Hz}, 0.8 \mathrm{H}, \mathrm{SCH}_{2} \mathrm{CH}_{2} \mathrm{CH}_{2} \mathrm{OH}\right.$ major), $4.55(\mathrm{t}$,
$J=5.2 \mathrm{~Hz}, 0.2 \mathrm{H}, \mathrm{SCH}_{2} \mathrm{CH}_{2} \mathrm{CH}_{2} \mathrm{OH}$ minor), $4.58(\mathrm{t}, J=5.1 \mathrm{~Hz}, 1 \mathrm{H}$, $\left.\mathrm{OCH}_{2} \mathrm{CH}_{2} \mathrm{OH}\right), 4.67\left(\mathrm{~m}, 1 \mathrm{H}, 3 \mathrm{a}{ }^{\prime \prime}-\mathrm{H}\right), 5.02\left(\mathrm{~m}, 1 \mathrm{H}, 6^{\prime \prime \prime}-\mathrm{H}\right), 5.18(\mathrm{~m}$, $0.2 \mathrm{H}, 6 \mathrm{a}$ "'- $H$ minor), 5.22 (dd, $J=7.2 \mathrm{~Hz} / 4.9 \mathrm{~Hz}, 0.8 \mathrm{H}, 6 \mathrm{a}$ '"- $H$ major), $7.02\left(\mathrm{~m}, 0.2 \mathrm{H}, 6^{\prime \prime}-H\right.$ minor), $7.08\left(\mathrm{~m}, 0.8 \mathrm{H}, 6^{\prime \prime}-\mathrm{H}\right.$ major), 7.22-7.36 (m, $\left.2 \mathrm{H}, 2^{\prime \prime}-H / 5^{\prime \prime}-H\right), 8.99$ (d, $J=4.6 \mathrm{~Hz}, 0.2 \mathrm{H}, \mathrm{NH}$ minor), 9.37 (d, $J=3.8 \mathrm{~Hz}, 0.8 \mathrm{H}, \mathrm{NH}$ major). ${ }^{13} \mathrm{C}$ NMR (DMSO- $\left.d_{6}\right) \delta 15.1,23.9,24.8$, $26.9,27.4,32.3,33.9,35.5,59.3,60.1,61.5,70.7,81.9,82.1,83.7,112.5$, $115.0,117.1,123.0,123.2,139.2,146.9,148.4,148.8,149.1,150.4,154.0$, 169.5 .

4.61. (1S,2R,3S,4R)-4-(7-(((1R,2S)-2-(3,4-difluorophenyl) cyclopropyl)amino)-3H-[1,2,3]triazolo[4,5-d]pyrimidin-3-yl) cyclopentane-1,2,3-triol (7u)

To a solution of $15 \mathrm{c} .1$ ( $0.15 \mathrm{~g}, 0.34 \mathrm{mmol})$ in $\mathrm{MeOH}(3 \mathrm{~mL})$, was added $\mathrm{HCl} 12 \mathrm{~N}(1 \mathrm{~mL})$. After $30 \mathrm{~min}$ at room temperature, methanol was evaporated under reduced pressure and the resulting residue was suspended in water $(20 \mathrm{~mL})$, neutralized with $\mathrm{NaOH}$ $10 \%$ and extracted with dichloromethane $(3 \times 20 \mathrm{~mL})$. The combined organic layers were dried over $\mathrm{MgSO}_{4}$, concentrated under reduced pressure and purified by column chromatography on silica gel using ethyl acetate $100 \%$ to give $7 \mathbf{u}$ as a white solid $(0.12 \mathrm{~g}, 88 \%$ yield, m.p.: $\left.148-152{ }^{\circ} \mathrm{C}\right) .{ }^{1} \mathrm{H}$ NMR (DMSO- $\left.d_{6}\right) \delta 1.37$ (m, 0.8H, $3^{\prime}-\mathrm{Ha}$ major), 1.41 ( $\mathrm{m}, 0.2 \mathrm{H}, 3^{\prime}-\mathrm{Ha}$ minor), 1.49 ( $\mathrm{m}, 0.2 \mathrm{H}, 3^{\prime}-\mathrm{Hb}$ minor), 1.54 $\left(\mathrm{m}, 0.8 \mathrm{H}, 3^{\prime}-\mathrm{Hb}\right.$ major), $1.94\left(\mathrm{~m}, 1 \mathrm{H}, 5^{\prime \prime \prime}-\mathrm{Ha}\right), 2.19\left(\mathrm{~m}, 0.8 \mathrm{H}, 2^{\prime}-\mathrm{H}\right.$ major), $2.26\left(\mathrm{~m}, 0.2 \mathrm{H}, 2^{\prime}-H\right.$ minor), $2.63\left(\mathrm{~m}, 1 \mathrm{H}, 5^{\prime \prime \prime}-H b\right), 3.29(\mathrm{~m}$, $0.8 \mathrm{H}, 1^{\prime}-\mathrm{H}$ major), $3.80\left(\mathrm{~m}, 1 \mathrm{H}, 3^{\prime \prime \prime}-\mathrm{H}\right), 3.82\left(\mathrm{~m}, 0.2 \mathrm{H}, 1^{\prime}-\mathrm{H}\right.$ minor $)$, $3.94\left(\mathrm{~m}, 1 \mathrm{H}, 1^{\prime \prime \prime}-H\right), 4.67\left(\mathrm{~m}, 1 \mathrm{H}, 2^{\prime \prime \prime}-H\right), 4.93\left(\mathrm{~d}, J=3.6 \mathrm{~Hz}, 1 \mathrm{H}, 3^{\prime \prime \prime}-\right.$ $\mathrm{OH}), 5.06\left(\mathrm{~m}, 2 \mathrm{H}, 2^{\prime \prime \prime}-\mathrm{OH}, 4^{\prime \prime \prime}-\mathrm{H}\right), 5.16\left(\mathrm{~d}, J=5.2 \mathrm{~Hz}, 1 \mathrm{H}, 1^{\prime \prime \prime}-\mathrm{OH}\right), 7.05$ ( $\mathrm{m}, 0.2 \mathrm{H}, 6^{\prime \prime}-H$ minor), 7.09 ( $\mathrm{m}, 0.8 \mathrm{H}, 6^{\prime \prime}-H$ major), 7.27-7.36 $(\mathrm{m}, 2 \mathrm{H}$, $2^{\prime \prime}-H / 5^{\prime \prime}-H$ ), 8.30 (s, 0.2H, 2- $H$ minor), 8.40 (s, 0.8H, 2- $H$ major), 8.95 (s, 0.2H, NH minor), 9.29 (d, $J=4.0 \mathrm{~Hz}, 0.8 \mathrm{H}, \mathrm{NH}$ major). ${ }^{13} \mathrm{C}$ NMR $\left(\right.$ DMSO- $\left.d_{6}\right) \delta 15.3,23.7,33.9,36.1,59.8,61.2,73.3,74.9,76.8,114.9$, 117.2, 123.0, 124.6, 139.3, 147.1, 148.4, 148.5, 148.7, 150.1, 154.8, 156.1, 156.2. Anal. $\left(\mathrm{C}_{18} \mathrm{H}_{18} \mathrm{~F}_{2} \mathrm{~N}_{6} \mathrm{O}_{3} . \mathrm{H}_{2} \mathrm{O}\right)$ theoretical: $\mathrm{C}, 51.18 ; \mathrm{H}, 4.77 ; \mathrm{N}$, 19.90. Found: C, 51.23; H, 4.74; N, 19.60.

4.62. (1S,2R,3S,4R)-4-(5-chloro-7-(((1R,2S)-2-(3,4-difluorophenyl) cyclopropyl)amino)-3H-[1,2,3]triazolo[4,5-d]pyrimidin-3-yl) cyclopentane-1,2,3-triol (7v)

To a solution of 15a.2 $(0.15 \mathrm{~g}, 0.31 \mathrm{mmol})$ in $\mathrm{MeOH}(3 \mathrm{~mL})$, was added $\mathrm{HCl} 12 \mathrm{~N}(1 \mathrm{~mL})$. After $30 \mathrm{~min}$ at room temperature, methanol was evaporated under reduced pressure and the resulting residue was suspended in water $(20 \mathrm{~mL})$, neutralized with $\mathrm{NaOH}$ $10 \%$ and extracted with dichloromethane $(3 \times 20 \mathrm{~mL})$. The combined organic layers were dried over $\mathrm{MgSO}_{4}$, concentrated under reduced pressure and purified by column chromatography on silica gel using ethyl acetate $100 \%$ to give $7 \mathbf{v}$ as a white solid $(0.11 \mathrm{~g}, 80 \%$ yield, m.p.: $\left.195-200{ }^{\circ} \mathrm{C}\right) .{ }^{1} \mathrm{H}$ NMR (DMSO- $\left.d_{6}\right) \delta 1.42-1.50\left(\mathrm{~m}, 2 \mathrm{H}, 3^{\prime}-\right.$ $H$ ), $1.89\left(\mathrm{~m}, 1 \mathrm{H}, 5^{\prime \prime \prime}-\mathrm{Ha}\right.$ ), 2.18 (ddd, $J=9.6 \mathrm{~Hz} / 6.5 \mathrm{~Hz} / 3.3 \mathrm{~Hz}, 0.7 \mathrm{H}, 2^{\prime}-$ $H$ major), 2.29 (ddd, $J=9.6 \mathrm{~Hz} / 6.4 \mathrm{~Hz} / 3.2 \mathrm{~Hz}, 0.3 \mathrm{H}, 2^{\prime}-H$ minor), 2.63 (m, $\left.1 \mathrm{H}, 5^{\prime \prime \prime}-H b\right), 3.14\left(\mathrm{~m}, 0.7 \mathrm{H}, 1^{\prime}-H\right.$ major), $3.77\left(\mathrm{~m}, 1 \mathrm{H}, 3^{\prime \prime \prime}-H\right), 3.82$ (m, 0.3H, $1^{\prime}-H$ minor), $3.94\left(\mathrm{~m}, 1 \mathrm{H}, 1^{\prime \prime \prime}-H\right), 4.56\left(\mathrm{~m}, 0.3 \mathrm{H}, 2^{\prime \prime \prime}-H \mathrm{mi}-\right.$ nor), $4.61\left(\mathrm{~m}, 0.7 \mathrm{H}, 2^{\prime \prime \prime}-H\right.$ major $), 4.96\left(\mathrm{~m}, 2 \mathrm{H}, 3^{\prime \prime \prime}-\mathrm{OH} / 4^{\prime \prime \prime}-\mathrm{H}\right), 5.05(\mathrm{~d}$, $J=6.4 \mathrm{~Hz}, 0.3 \mathrm{H}, 2^{\prime \prime \prime}-\mathrm{OH}$ minor), 5.08 (d, $J=6.4 \mathrm{~Hz}, 0.7 \mathrm{H}, 2^{\prime \prime \prime}-\mathrm{OH}$ major), 5.13 (d, $J=4.1 \mathrm{~Hz}, 0.3 \mathrm{H}, 1^{\prime \prime \prime}-\mathrm{OH}$ minor), 5.15 (d, $J=4.0 \mathrm{~Hz}$, $0.7 \mathrm{H}, 1^{\prime \prime \prime}-\mathrm{OH}$ major), 7.04 (m, 0.3H, 6" $-\mathrm{H}$ minor), 7.14 (m, 0.7H, 6" $-\mathrm{H}$ major), 7.24-7.42 (m, 2H, 2" $\left.-H / 5^{\prime \prime}-H\right), 9.53(\mathrm{~d}, J=5.0 \mathrm{~Hz}, 0.3 \mathrm{H}, \mathrm{NH}$ minor), 9.81 (d, $J=3.9 \mathrm{~Hz}, 0.7 \mathrm{H}, \mathrm{NH}$ major). ${ }^{13} \mathrm{C}$ NMR (DMSO-d $d_{6}$ ) $\delta$ 14.6, 23.7, 24.4, 33.6, 35.6, 36.1, 36.2, 61.0, 73.2, 75.1, 76.7, 114.9, $115.6,117.1,122.9,123.5,123.6,124.0,138.6,147.2,148.6,150.0,150.1$, 151.7, 155.4, 156.6, 156.8, 157.6. Anal. $\left(\mathrm{C}_{18} \mathrm{H}_{17} \mathrm{ClF}_{2} \mathrm{~N}_{6} \mathrm{O}_{3} . \mathrm{H}_{2} \mathrm{O}\right)$ theoretical: C, 47.32; H, 4.19; N, 18.40. Found: C, 47.51; H, 4.07; N, 18.08. 
4.63. (1S,2R,3S,4R)-4-(7-amino-5-(propylthio)-3H-[1,2,3]triazolo [4,5-d]pyrimidin-3-yl)cyclopentane-1,2,3-triol (7h)

To a solution of $\mathbf{1 6 a}(0.15 \mathrm{~g}, 0.41 \mathrm{mmol})$ in $\mathrm{MeOH}(3 \mathrm{~mL})$, was added $\mathrm{HCl} 12 \mathrm{~N}(1 \mathrm{~mL})$. After $30 \mathrm{~min}$ at room temperature, methanol was evaporated under reduced pressure and the resulting residue was suspended in water $(20 \mathrm{~mL})$, neutralized with $\mathrm{NaOH}$ $10 \%$ and extracted with dichloromethane $(3 \times 20 \mathrm{~mL})$. The combined organic layers were dried over $\mathrm{MgSO}_{4}$, concentrated under reduced pressure and purified by column chromatography on silica gel using ethyl acetate/methanol gradient to give $\mathbf{7 h}$ as a white solid (0.05 g, 37\% yield, m.p.: $\left.175-180{ }^{\circ} \mathrm{C}\right) .{ }^{1} \mathrm{H}$ NMR (DMSO- $\left.d_{6}\right) \delta 0.99(\mathrm{t}$, $\left.J=7.3 \mathrm{~Hz}, 3 \mathrm{H}, \mathrm{SCH}_{2} \mathrm{CH}_{2} \mathrm{CH}_{3}\right), 1.68\left(\mathrm{~h}, J=7.1 \mathrm{~Hz}, 2 \mathrm{H}, \mathrm{SCH}_{2} \mathrm{CH}_{2} \mathrm{CH}_{3}\right)$, 1.99 (ddd, $J=13.5 \mathrm{~Hz} / 9.1 \mathrm{~Hz} / 4.4 \mathrm{~Hz}, 1 \mathrm{H}, 5^{\prime}-\mathrm{Ha}$ ), 2.58 (ddd, $\left.J=13.7 \mathrm{~Hz} / 9.1 \mathrm{~Hz} / 7.2 \mathrm{~Hz}, 1 \mathrm{H}, 5^{\prime}-\mathrm{Hb}\right), 3.08\left(\mathrm{~m}, 2 \mathrm{H}, \mathrm{SCH}_{2} \mathrm{CH}_{2} \mathrm{CH}_{3}\right)$, $3.78\left(\mathrm{~m}, 1 \mathrm{H}, 2^{\prime}-H\right), 3.93\left(\mathrm{~m}, 1 \mathrm{H}, 1^{\prime}-H\right), 4.68\left(\mathrm{~m}, 1 \mathrm{H}, 3^{\prime}-H\right), 4.96(\mathrm{q}$, $J=9.0 \mathrm{~Hz}, 1 \mathrm{H}, 4^{\prime}-\mathrm{H}$ ), 5.08 (bs, $3 \mathrm{H}, 1^{\prime \prime \prime}-\mathrm{OH} / 2^{\prime \prime \prime}-\mathrm{OH} / 3^{\prime \prime \prime}-\mathrm{OH}$ ), 8.05 (bs, $1 \mathrm{H}, \mathrm{NHa}), 8.40$ (bs, $1 \mathrm{H}, \mathrm{NHb}) .{ }^{13} \mathrm{C} \mathrm{NMR}\left(\mathrm{CDCl}_{3}\right) \delta 13.3,22.5,32.1,35.8$, 61.2, 73.2, 74.3, 76.8, 122.8, 149.6, 155.1, 169.0. Anal. $\left(\mathrm{C}_{12} \mathrm{H}_{18} \mathrm{~N}_{6} \mathrm{O}_{3} \mathrm{~S} . \mathrm{H}_{2} \mathrm{O}\right)$ theoretical: $\mathrm{C}, 41.85 ; \mathrm{H}, 5.85 ; \mathrm{N}, 24.40$. Found: $\mathrm{C}$, $41.83 ; \mathrm{H}, 5.51$; N, 24.18.

4.64. (1S,2R,3S,4R)-4-(7-(((1R,2S)-2-(3,4-difluorophenyl) cyclopropyl)amino)-5-(propylthio)-3H-[1,2,3]triazolo[4,5-d] pyrimidin-3-yl)cyclopentane-1,2,3-triol (7i)

To a solution of $\mathbf{1 6 b}(0.15 \mathrm{~g}, 0.29 \mathrm{mmol})$ in $\mathrm{MeOH}(3 \mathrm{~mL})$, was added $\mathrm{HCl} 12 \mathrm{~N}(1 \mathrm{~mL})$. After $30 \mathrm{~min}$ at room temperature, methanol was evaporated under reduced pressure and the resulting residue was suspended in water $(20 \mathrm{~mL})$, neutralized with $\mathrm{NaOH}$ $10 \%$ and extracted with dichloromethane $(3 \times 20 \mathrm{~mL})$. The combined organic layers were dried over $\mathrm{MgSO}_{4}$, concentrated under reduced pressure and purified by column chromatography on silica gel using ethyl acetate $100 \%$ to give $7 \mathbf{i}$ as a white solid $(0.104 \mathrm{~g}, 75 \%$ yield, m.p.: $60-65^{\circ} \mathrm{C}$ ). ${ }^{1} \mathrm{H}$ NMR (DMSO- $\left.d_{6}\right) \delta 0.81(\mathrm{t}, J=7.4 \mathrm{~Hz}, 2.4 \mathrm{H}$, $\mathrm{SCH}_{2} \mathrm{CH}_{2} \mathrm{CH}_{3}$ major), 0.99 (t, $J=7.3 \mathrm{~Hz}, 0.6 \mathrm{H}, \mathrm{SCH}_{2} \mathrm{CH}_{2} \mathrm{CH}_{3}$ minor), 1.38 (dq, $J=13.8 \mathrm{~Hz} / 6.4 \mathrm{~Hz}, 1 \mathrm{H}, 3^{\prime}-\mathrm{Ha}$ ), 1.49 (tq, $J=13.6 \mathrm{~Hz} / 6.9 \mathrm{~Hz}$, $1.8 \mathrm{H}, \mathrm{SCH}_{2} \mathrm{CH}_{2} \mathrm{CH}_{3}$ major/3'- $\mathrm{Hb}$ minor), 1.56 (dt, $J=10.2 \mathrm{~Hz} / 5.5 \mathrm{~Hz}$, $0.8 \mathrm{H}, 3^{\prime}-\mathrm{Hb}$ major), 1.68 ( $\mathrm{h}, J=7.1 \mathrm{~Hz}, 0.4 \mathrm{H}, \mathrm{SCH}_{2} \mathrm{CH}_{2} \mathrm{CH}_{3}$ minor), 1.91 (ddd, $J=13.6 \mathrm{~Hz} / 9.0 \mathrm{~Hz} / 4.3 \mathrm{~Hz}, 1 \mathrm{H}, 5^{\prime \prime \prime}-\mathrm{Ha}$ ), 2.13 (ddd, $J=9.5 \mathrm{~Hz} / 6.3 \mathrm{~Hz} / 3.5 \mathrm{~Hz}, 0.8 \mathrm{H}, 2^{\prime}-\mathrm{H}$ major), 2.25 (m, 0.2H, $2^{\prime}-\mathrm{H}$ minor), 2.59 (ddd, $J=13.7 \mathrm{~Hz} / 9.1 \mathrm{~Hz} / 7.3 \mathrm{~Hz}, 1 \mathrm{H}, 5^{\prime \prime \prime}-\mathrm{Hb}$ ), 2.85 (dt, $J=13.6 \mathrm{~Hz} / 7.2 \mathrm{~Hz}, 0.8 \mathrm{H}$, SCHa major), $2.95(\mathrm{dt}, J=13.9 \mathrm{~Hz} / 7.1 \mathrm{~Hz}$, $0.8 \mathrm{H}, \mathrm{SCH} b$ major), 3.09 ( $\mathrm{m}, 0.4 \mathrm{H}, \mathrm{SCH} 2$ minor), $3.16\left(\mathrm{~m}, 0.8 \mathrm{H}, 1^{\prime}-H\right.$ major), $3.78\left(\mathrm{~m}, 1.2 \mathrm{H}, 1^{\prime}-H\right.$ minor $\left./ 2^{\prime \prime \prime}-H\right), 3.93(\mathrm{dt}, J=6.7 \mathrm{~Hz} / 3.3 \mathrm{~Hz}$, $\left.1 \mathrm{H}, 1^{\prime \prime \prime}-H\right), 4.65\left(\mathrm{~m}, 1 \mathrm{H}, 3^{\prime \prime \prime}-H\right), 4.91\left(\mathrm{~d}, J=3.9 \mathrm{~Hz}, 1 \mathrm{H}, 2^{\prime \prime \prime}-\mathrm{OH}\right), 4.95$ $\left(\mathrm{q}, J=9.0 \mathrm{~Hz}, 1 \mathrm{H}, 4^{\prime \prime \prime}-H\right), 4.99$ (d, $J=6.5 \mathrm{~Hz}, 0.2 \mathrm{H}, 3^{\prime \prime \prime}-\mathrm{OH}$ minor), $5.02\left(\mathrm{~d}, J=6.4 \mathrm{~Hz}, 0.8 \mathrm{H}, 3^{\prime \prime \prime}-\mathrm{OH}\right.$ major), $5.07\left(\mathrm{~d}, J=3.8 \mathrm{~Hz}, 0.2 \mathrm{H}, 1^{\prime \prime \prime}-\right.$ $\mathrm{OH}$ minor), 5.10 (d, $J=4.1 \mathrm{~Hz}, 0.8 \mathrm{H}, 1^{\prime \prime \prime}-\mathrm{OH}$ major), 7.03 (bs, $0.2 \mathrm{H}, 6^{\prime \prime}-$ $H$ minor), 7.07 (bs, 0.8H, 6" $-H$ major), 7.24-7.36 (m, 2H, $2^{\prime \prime}-H / 5^{\prime \prime}-H$ ), $8.93(\mathrm{~d}, J=4.7 \mathrm{~Hz}, 0.2 \mathrm{H}, \mathrm{NH}$ minor), 9.35 (d, $J=4.0 \mathrm{~Hz}, 0.8 \mathrm{H}, \mathrm{NH}$ major). ${ }^{13} \mathrm{C}$ NMR (DMSO-d $\left.d_{6}\right) \delta 13.0,15.0,22.3,24.0,32.3,34.1,36.0$, 61.0, 73.2, 74.5, 76.8, 114.8, 117.0, 122.8, 123.1, 139.3, 147.1, 148.5, 149.4, 150.1, 153.9, 169.1. Anal. $\left(\mathrm{C}_{21} \mathrm{H}_{24} \mathrm{~F}_{2} \mathrm{~N}_{6} \mathrm{O}_{3} \mathrm{~S}\right)$ theoretical: $\mathrm{C}$, 52.71; H, 5.06; N, 17.56; S, 6.70. Found: C, 52.31; H, 5.26; N, 17.50, S, 6.28 .

4.65. (1S,2S,3R,5S)-3-(7-amino-5-(propylthio)-3H-[1,2,3]triazolo [4,5-d]pyrimidin-3-yl)-5-(2-hydroxyethoxy)cyclopentane-1,2-diol (7j)

To a solution of $\mathbf{1 6 c}(0.15 \mathrm{~g}, 0.37 \mathrm{mmol})$ in $\mathrm{MeOH}(3 \mathrm{~mL})$, was added $\mathrm{HCl} 12 \mathrm{~N}(1 \mathrm{~mL})$. After $30 \mathrm{~min}$ at room temperature, methanol was evaporated under reduced pressure and the resulting residue was suspended in water $(20 \mathrm{~mL})$, neutralized with $\mathrm{NaOH}$
$10 \%$ and extracted with dichloromethane $(3 \times 20 \mathrm{~mL})$. The combined organic layers were dried over $\mathrm{MgSO}_{4}$, concentrated under reduced pressure and purified by column chromatography on silica gel using ethyl acetate/methanol gradient to give $\mathbf{7} \mathbf{j}$ as a white solid $\left(0.09\right.$ g, 66\% yield, m.p.: $\left.180-182{ }^{\circ} \mathrm{C}\right) .{ }^{1} \mathrm{H}$ NMR (DMSO- $\left.d_{6}\right) \delta 0.99(\mathrm{t}$, $\left.J=7.3 \mathrm{~Hz}, 3 \mathrm{H}, \mathrm{SCH}_{2} \mathrm{CH}_{2} \mathrm{CH}_{3}\right), 1.69$ (h, $J=7.2 \mathrm{~Hz}, 2 \mathrm{H}, \mathrm{SCH}_{2} \mathrm{CH}_{2} \mathrm{CH}_{3}$ ), 2.06 (ddd, $J=14.3 \mathrm{~Hz} / 9.8 \mathrm{~Hz} / 5.0 \mathrm{~Hz}, 1 \mathrm{H}, 4^{\prime}-\mathrm{Ha}$ ), $2.63\left(\mathrm{~m}, 1 \mathrm{H}, 4^{\prime}-\mathrm{Hb}\right.$ ), $3.08\left(\mathrm{~m}, 2 \mathrm{H}, \mathrm{SCH}_{2} \mathrm{CH}_{2} \mathrm{CH}_{3}\right), 3.46-3.52\left(\mathrm{~m}, 4 \mathrm{H}, \mathrm{OCH}_{2} \mathrm{CH}_{2} \mathrm{OH}\right), 3.76(\mathrm{t}$, $\left.J=6.1 \mathrm{~Hz}, 1 \mathrm{H}, 5^{\prime}-H\right), 3.94\left(\mathrm{~m}, 1 \mathrm{H}, 1^{\prime}-H\right), 4.60\left(\mathrm{~m}, 2 \mathrm{H}, 2^{\prime}-\mathrm{H} / \mathrm{OCH}_{2-}\right.$ $\left.\mathrm{CH}_{2} \mathrm{OH}\right), 4.97\left(\mathrm{q}, J=9.1 \mathrm{~Hz}, 1 \mathrm{H}, 3^{\prime}-H\right), 5.06\left(\mathrm{~d}, J=3.9 \mathrm{~Hz}, 1 \mathrm{H}, 1^{\prime}-\mathrm{OH}\right)$, $5.13\left(\mathrm{~d}, J=6.4 \mathrm{~Hz}, 1 \mathrm{H}, 2^{\prime}-\mathrm{OH}\right), 8.07(\mathrm{~s}, 1 \mathrm{H}, \mathrm{NHa}), 8.42(\mathrm{~s}, 1 \mathrm{H}, \mathrm{NHb}) .{ }^{13} \mathrm{C}$ NMR (DMSO- $\left.d_{6}\right) \delta 13.3,22.4,32.1,33.1,60.3,60.7,70.8,73.7,74.1$, 81.7, 122.8, 149.6, 155.1, 169.0. Anal. $\left(\mathrm{C}_{14} \mathrm{H}_{22} \mathrm{~N}_{6} \mathrm{O}_{4} \mathrm{~S}\right)$ theoretical: $\mathrm{C}$, 45.39; H, 5.99; N, 22.69; S, 8.66. Found: C, 45.66; H, 6.33; N, 22.53, S, 8.58 .

4.66. (1S,2S,3R,5S)-3-(7-(Cyclopropylamino)-5-(propylthio)-3H[1,2,3]triazolo[4,5-d]pyrimidin-3-yl)-5-(2-hydroxyethoxy) cyclopentane-1,2-diol (7k)

To a solution of $\mathbf{1 6 d}(0.15 \mathrm{~g}, 0.33 \mathrm{mmol})$ in $\mathrm{MeOH}(3 \mathrm{~mL})$, was added $\mathrm{HCl} 12 \mathrm{~N}(1 \mathrm{~mL})$. After $30 \mathrm{~min}$ at room temperature, methanol was evaporated under reduced pressure and the resulting residue was suspended in water $(20 \mathrm{~mL})$, neutralized with $\mathrm{NaOH}$ $10 \%$ and extracted with dichloromethane $(3 \times 20 \mathrm{~mL})$. The combined organic layers were dried over $\mathrm{MgSO}_{4}$, concentrated under reduced pressure and purified by column chromatography on silica gel using ethyl acetate/methanol gradient to give $7 \mathbf{k}$ as a white solid $\left(0.09 \mathrm{~g}, 66 \%\right.$ yield, m.p.: $\left.156-158^{\circ} \mathrm{C}\right) .{ }^{1} \mathrm{H}$ NMR (DMSO-d $\left.d_{6}\right) \delta 0.69(\mathrm{~m}$, $\left.2 \mathrm{H}, 2^{\prime}-\mathrm{Ha} / 3^{\prime}-\mathrm{Ha}\right), 0.76\left(\mathrm{~m}, 1.4 \mathrm{H}, 2^{\prime}-\mathrm{Hb}\right.$ major $/ 3^{\prime}-\mathrm{Hb}$ major$), 0.87(\mathrm{~m}$, $0.6 \mathrm{H}, 2^{\prime}-\mathrm{Hb}$ minor $/ 3^{\prime}-\mathrm{Hb}$ minor), $0.99(\mathrm{t}, J=7.3 \mathrm{~Hz}, 3 \mathrm{H}$, $\left.\mathrm{SCH}_{2} \mathrm{CH}_{2} \mathrm{CH}_{3}\right), 1.71\left(\mathrm{~m}, 2 \mathrm{H}, \mathrm{SCH}_{2} \mathrm{CH}_{2} \mathrm{CH}_{3}\right), 2.04$ (ddd, $J=14.1 \mathrm{~Hz} /$ $\left.9.8 \mathrm{~Hz} / 5.0 \mathrm{~Hz}, 1 \mathrm{H}, 4^{\prime \prime}-\mathrm{Ha}\right), 2.65\left(\mathrm{~m}, 1 \mathrm{H}, 4^{\prime \prime}-\mathrm{Hb}\right), 3.05\left(\mathrm{~m}, 0.7 \mathrm{H}, 1^{\prime}-\mathrm{H}\right.$ major), $3.12\left(\mathrm{~m}, 2 \mathrm{H}, \mathrm{SCH}_{2} \mathrm{CH}_{2} \mathrm{CH}_{3}\right), 3.42-3.52\left(\mathrm{~m}, 4.3 \mathrm{H}, 1^{\prime}-\mathrm{H}\right.$ minor/ $\left.\mathrm{OCH}_{2} \mathrm{CH}_{2} \mathrm{OH}\right), 3.75\left(\mathrm{~m}, 1 \mathrm{H}, 5^{\prime \prime}-\mathrm{H}\right), 3.94\left(\mathrm{~m}, 1 \mathrm{H}, 1^{\prime \prime}-\mathrm{H}\right), 4.56\left(\mathrm{~m}, 1 \mathrm{H}, 2^{\prime \prime}-\right.$ $H), 4.61\left(\mathrm{~m}, 1 \mathrm{H}, \mathrm{OCH}_{2} \mathrm{CH}_{2} \mathrm{OH}\right), 4.96\left(\mathrm{~m}, 1 \mathrm{H}, 3^{\prime \prime}-\mathrm{H}\right), 5.06$ (bs, $1 \mathrm{H}, 1^{\prime \prime}-$ $\mathrm{OH}$ ), 5.13 (bs, $1 \mathrm{H}, 2^{\prime \prime}-\mathrm{OH}$ ), 8.78 (d, $J=2.6 \mathrm{~Hz}, 0.3 \mathrm{H}, \mathrm{NH}$ minor), 9.08 (d, $J=2.1 \mathrm{~Hz}, 0.7 \mathrm{H}, \mathrm{NH}$ major). ${ }^{13} \mathrm{C}$ NMR (DMSO- $\left.d_{6}\right) \delta 5.9,7.5,13.3$, 22.7, 23.7, 24.7, 26.1, 32.5, 33.2, 60.3, 61.5, 70.8, 73.6, 74.0, 74.3, 81.8, 123.1, 149.2, 154.1, 169.1. Anal. $\left(\mathrm{C}_{17} \mathrm{H}_{26} \mathrm{~N}_{6} \mathrm{O}_{4} \mathrm{~S}\right)$ theoretical: $\mathrm{C}, 49.74$; H, 6.38; N, 20.47; S, 7.81. Found: C, 49.90; H, 6.44; N, 20.57, S, 7.10.

4.67. (1S,2S,3R,5S)-3-(7-(((1R,2S)-2-phenylcyclopropyl)amino)-5(propylthio)-3H-[1,2,3]triazolo[4,5-d]pyrimidin-3-yl)-5-(2hydroxyethoxy)-cyclopentane-1,2-diol (7l)

To a solution of $16 \mathbf{e}(0.15 \mathrm{~g}, 0.28 \mathrm{mmol})$ in $\mathrm{MeOH}(3 \mathrm{~mL})$, was added $\mathrm{HCl} 12 \mathrm{~N}(1 \mathrm{~mL})$. After $30 \mathrm{~min}$ at room temperature, methanol was evaporated under reduced pressure and the resulting residue was suspended in water $(20 \mathrm{~mL})$, neutralized with $\mathrm{NaOH}$ $10 \%$ and extracted with dichloromethane $(3 \times 20 \mathrm{~mL})$. The combined organic layers were dried over $\mathrm{MgSO}_{4}$, concentrated under reduced pressure and purified by column chromatography on silica gel using ethyl acetate $100 \%$ to give 71 as a white solid $(0.11 \mathrm{~g}, 79 \%$ yield, m.p.: $\left.174-179{ }^{\circ} \mathrm{C}\right) .{ }^{1} \mathrm{H}$ NMR (DMSO- $\left.d_{6}\right) \delta 0.80(\mathrm{t}, J=7.4 \mathrm{~Hz}$, $2.4 \mathrm{H}, \mathrm{SCH}_{2} \mathrm{CH}_{2} \mathrm{CH}_{3}$ major), 0.99 (t, $J=7.3 \mathrm{~Hz}, 0.6 \mathrm{H}, \mathrm{SCH}_{2} \mathrm{CH}_{2} \mathrm{CH}_{3}$ minor), $1.32\left(\mathrm{~m}, 1 \mathrm{H}, 3^{\prime}-\mathrm{Ha}\right), 1.40\left(\mathrm{~m}, 0.2 \mathrm{H}, 3^{\prime}-\mathrm{Hb}\right.$ minor), $1.50(\mathrm{~m}$, $2.4 \mathrm{H}, 3^{\prime}-\mathrm{Hb}$ major $/ \mathrm{SCH}_{2} \mathrm{CH}_{2} \mathrm{CH}_{3}$ major), 1.69 (h, $J=7.1 \mathrm{~Hz}, 0.4 \mathrm{H}$, $\mathrm{SCH}_{2} \mathrm{CH}_{2} \mathrm{CH}_{3}$ minor), 2.04 (ddd, $J=14.1 \mathrm{~Hz} / 9.7 \mathrm{~Hz} / 5.0 \mathrm{~Hz}, 1 \mathrm{H}, 4^{\prime \prime \prime}-$ $\mathrm{Ha}$ ), 2.12 (ddd, $J=9.5 \mathrm{~Hz} / 6.3 \mathrm{~Hz} / 3.3 \mathrm{~Hz}, 0.8 \mathrm{H}, 2^{\prime}-H$ major), 2.26 (m, $0.2 \mathrm{H}, 2^{\prime}-\mathrm{H}$ minor), $2.63\left(\mathrm{~m}, 1 \mathrm{H}, 4^{\prime \prime \prime}-\mathrm{Hb}\right), 2.88\left(\mathrm{~m}, 1.6 \mathrm{H}, \mathrm{SCH}_{2} \mathrm{CH}_{2} \mathrm{CH}_{3}\right.$ major), $3.09\left(\mathrm{~m}, 0.4 \mathrm{H}, \mathrm{SCH}_{2} \mathrm{CH}_{2} \mathrm{CH}_{3}\right.$ minor), $3.20(\mathrm{dq}, J=7.9 \mathrm{~Hz}$ ) $4.4 \mathrm{~Hz}, 0.8 \mathrm{H}, 1^{\prime}-\mathrm{H}$ major), $3.45-3.53\left(\mathrm{~m}, 4 \mathrm{H}, \mathrm{OCH}_{2} \mathrm{CH}_{2} \mathrm{OH}\right), 3.75(\mathrm{~m}$, $\left.1 \mathrm{H}, 5^{\prime \prime \prime}-H\right), 3.85\left(\mathrm{~m}, 0.2 \mathrm{H}, 1^{\prime}-H\right.$ minor), $3.93\left(\mathrm{~m}, 1 \mathrm{H}, 1^{\prime \prime \prime}-H\right), 4.54(\mathrm{~m}$, $\left.1 \mathrm{H}, 2^{\prime \prime \prime}-H\right), 4.61\left(\mathrm{t}, J=5.0 \mathrm{~Hz}, 1 \mathrm{H}, \mathrm{OCH}_{2} \mathrm{CH}_{2} \mathrm{OH}\right), 4.96(\mathrm{q}, J=9.1 \mathrm{~Hz}$, 
$\left.1 \mathrm{H}, 3^{\prime \prime \prime}-H\right), 5.05\left(\mathrm{~d}, J=4.1 \mathrm{~Hz}, 1 \mathrm{H}, 1^{\prime \prime \prime}-\mathrm{OH}\right), 5.12\left(\mathrm{~d}, J=6.4 \mathrm{~Hz}, 1 \mathrm{H}, 2^{\prime \prime \prime}-\right.$ $\mathrm{OH}$ ), 7.18 (m, 3H, 2" $\left.-H / 4^{\prime \prime}-H / 6^{\prime \prime}-H\right), 7.29$ (t, $J=7.6 \mathrm{~Hz}, 2 \mathrm{H}, 3^{\prime \prime}-H / 5^{\prime \prime}-$ $H$ ), 8.96 (d, $J=4.9 \mathrm{~Hz}, 0.2 \mathrm{H}, \mathrm{NH}$ minor), 9.35 (d, $J=4.3 \mathrm{~Hz}, 0.8 \mathrm{H}, \mathrm{NH}$ major). ${ }^{13} \mathrm{C}$ NMR (DMSO- $\left.d_{6}\right) \delta 13.0,15.0,22.3,24.5,32.4,33.2,33.9$, $60.3,60.4,70.8,73.6,74.4,81.7,123.1,125.6,125.9,128.2,141.2$, 149.4, 153.8, 169.1. Anal. $\left(\mathrm{C}_{23} \mathrm{H}_{30} \mathrm{~N}_{6} \mathrm{O}_{4} \mathrm{~S}\right)$ theoretical: $\mathrm{C}, 56.77 ; \mathrm{H}$ 6.21 ; N, 17.27; S, 6.59. Found: C, 56.64; H, 6.20; N, 17.08, S, 6.26.

4.68. (1S,2S,3R,5S)-3-(7-((3,4-difluorophenethyl)amino)-5(propylthio)-3H-[1,2,3]triazolo[4,5-d]pyrimidin-3-yl)-5-(2hydroxyethoxy)cyclopentane-1,2-diol (7m)

To a solution of $\mathbf{1 6 f}(0.15 \mathrm{~g}, 0.27 \mathrm{mmol})$ in $\mathrm{MeOH}(3 \mathrm{~mL})$, was added $\mathrm{HCl} 12 \mathrm{~N}(1 \mathrm{~mL})$. After $30 \mathrm{~min}$ at room temperature, methanol was evaporated under reduced pressure and the resulting residue was suspended in water $(20 \mathrm{~mL})$, neutralized with $\mathrm{NaOH}$ $10 \%$ and extracted with dichloromethane $(3 \times 20 \mathrm{~mL})$. The combined organic layers were dried over $\mathrm{MgSO}_{4}$, concentrated under reduced pressure and purified by column chromatography on silica gel using ethyl acetate $100 \%$ to give $\mathbf{7 m}$ as a white solid $(0.10 \mathrm{~g}, 72 \%$ yield, m.p.: $137-142{ }^{\circ} \mathrm{C}$ ). ${ }^{1} \mathrm{H}$ NMR (DMSO-d $\left.d_{6}\right) \delta 0.97(\mathrm{t}, J=7.3 \mathrm{~Hz}, 3 \mathrm{H}$, $\mathrm{SCH}_{2} \mathrm{CH}_{2} \mathrm{CH}_{3}$ ), 1.69 (h, $J=6.9 \mathrm{~Hz}, 2 \mathrm{H}, \mathrm{SCH}_{2} \mathrm{CH}_{2} \mathrm{CH}_{3}$ ), 2.03 (ddd, $\left.J=14.0 \mathrm{~Hz} / 9.7 \mathrm{~Hz} / 4.9 \mathrm{~Hz}, 1 \mathrm{H}, 4^{\prime \prime \prime}-\mathrm{Ha}\right), 2.63\left(\mathrm{~m}, 1 \mathrm{H}, 4^{\prime \prime \prime}-\mathrm{Hb}\right), 2.94(\mathrm{t}$, $\left.J=7.1 \mathrm{~Hz}, 2 \mathrm{H}, 2^{\prime}-\mathrm{H}_{2}\right), 3.08\left(\mathrm{~m}, 2 \mathrm{H}, \mathrm{SCH}_{2} \mathrm{CH}_{2} \mathrm{CH}_{3}\right), 3.45-3.52(\mathrm{~m}, 4 \mathrm{H}$ $\left.\mathrm{OCH}_{2} \mathrm{CH}_{2} \mathrm{OH}\right), 3.74\left(\mathrm{~m}, 2.6 \mathrm{H}, 1^{\prime}-\mathrm{H}_{2}\right.$ major $\left./ 5^{\prime \prime \prime}-\mathrm{H}\right), 3.94\left(\mathrm{~m}, 1 \mathrm{H}, 1^{\prime \prime \prime}-\mathrm{H}\right)$, $4.10\left(\mathrm{~m}, 0.4 \mathrm{H}, 1^{\prime}-\mathrm{H}_{2}\right.$ minor), $4.55\left(\mathrm{~m}, 1 \mathrm{H}, 2^{\prime \prime \prime}-\mathrm{H}\right), 4.61(\mathrm{t}, J=5.0 \mathrm{~Hz}$, $\left.1 \mathrm{H}, \mathrm{OCH}_{2} \mathrm{CH}_{2} \mathrm{OH}\right), 4.96\left(\mathrm{q}, J=9.2 \mathrm{~Hz}, 1 \mathrm{H}, 3^{\prime \prime \prime}-H\right), 5.06(\mathrm{~d}, J=4.1 \mathrm{~Hz}$, $\left.1 \mathrm{H}, 1^{\prime \prime \prime}-\mathrm{OH}\right), 5.12\left(\mathrm{~d}, J=6.4 \mathrm{~Hz}, 1 \mathrm{H}, 2^{\prime \prime \prime}-\mathrm{OH}\right), 7.08\left(\mathrm{~m}, 0.8 \mathrm{H}, 6^{\prime \prime}-\mathrm{H}\right.$ major), 7.17 (m, 0.2H, 6" $-\mathrm{H}$ minor), 7.30-7.42 (m, 2H, $\left.2^{\prime \prime}-\mathrm{H} / 5^{\prime \prime}-\mathrm{H}\right)$, $8.70(\mathrm{t}, J=6.4 \mathrm{~Hz}, 0.2 \mathrm{H}, \mathrm{NH}$ minor $), 9.06(\mathrm{~d}, J=5.6 \mathrm{~Hz}, 0.8 \mathrm{H}, \mathrm{NH}$ major). ${ }^{13} \mathrm{C}$ NMR (DMSO-d $\left.d_{6}\right) \delta 13.3,22.6,32.4,33.2,33.5,41.1,60.2$, $60.5,70.8,73.6,74.3,81.7,117.1,117.5,123.0,125.4,137.1,147.1,148.2$, $148.9,149.3,150.1,150.6,153.0,169.0$. Anal. $\left(\mathrm{C}_{22} \mathrm{H}_{28} \mathrm{~F}_{2} \mathrm{~N}_{6} \mathrm{O}_{4} \mathrm{~S}\right)$ theoretical: C, 51.75; H, 5.53; N, 16.46; S, 6.28. Found: C, 51.35; H, 5.81; $\mathrm{N}, 16.86, \mathrm{~S}, 6.34$.

4.69. (1S,2S,3R,5S)-3-(7-(((1R,2S)-2-(3,4-difluorophenyl) cyclopropyl)amino)-5-(propylthio)-3H-[1,2,3]triazolo[4,5-d] pyrimidin-3-yl)-5-(2-hydroxyethoxy)cyclopentane-1,2-diol (1, ticagrelor)

To a solution of $\mathbf{1 6 g}(0.15 \mathrm{~g}, 0.27 \mathrm{mmol})$ in $\mathrm{MeOH}(3 \mathrm{~mL})$, was added $\mathrm{HCl} 12 \mathrm{~N}(1 \mathrm{~mL})$. After $30 \mathrm{~min}$ at room temperature, methanol was evaporated under reduced pressure and the resulting residue was suspended in water $(20 \mathrm{~mL})$, neutralized with $\mathrm{NaOH}$ $10 \%$ and extracted with dichloromethane $(3 \times 20 \mathrm{~mL})$. The combined organic layers were dried over $\mathrm{MgSO}_{4}$, concentrated under reduced pressure and purified by column chromatography on silica gel using ethyl acetate $100 \%$ to give 1 as a white solid $(0.115 \mathrm{~g}, 83 \%$ yield, m.p.: $135-138{ }^{\circ} \mathrm{C}$ ). ${ }^{1} \mathrm{H}$ NMR (DMSO- $\left.d_{6}\right) \delta 0.82(\mathrm{t}, J=7.3 \mathrm{~Hz}$, $2.4 \mathrm{H}, \mathrm{SCH}_{2} \mathrm{CH}_{2} \mathrm{CH}_{3}$ major), 0.99 (t, $J=7.3 \mathrm{~Hz}, 0.6 \mathrm{H}, \mathrm{SCH}_{2} \mathrm{CH}_{2} \mathrm{CH}_{3}$ minor), $1.37\left(\mathrm{~m}, 1 \mathrm{H}, 3^{\prime}-\mathrm{Ha}\right), 1.46\left(\mathrm{~m}, 0.2 \mathrm{H}, 3^{\prime}-\mathrm{Hb}\right.$ minor), 1.50 (ddt, $J=11.5 \mathrm{~Hz} / 7.1 \mathrm{~Hz} / 4.4 \mathrm{~Hz}, 1.6 \mathrm{H}, \mathrm{SCH}_{2} \mathrm{CH}_{2} \mathrm{CH}_{3}$ major), 1.55 (dt, $J=10.3 \mathrm{~Hz} / 5.4 \mathrm{~Hz}, 0.8 \mathrm{H}, 3^{\prime}-H b$ major $), 1.69(\mathrm{~h}, J=6.9 \mathrm{~Hz}, 0.4 \mathrm{H}$, $\mathrm{SCH}_{2} \mathrm{CH}_{2} \mathrm{CH}_{3}$ minor), $2.03\left(\mathrm{~m}, 1 \mathrm{H}, 4^{\prime \prime \prime}-\mathrm{Ha}\right), 2.12\left(\mathrm{~m}, 0.8 \mathrm{H}, 2^{\prime}-\mathrm{H}\right.$ major), $2.25\left(\mathrm{~m}, 0.2 \mathrm{H}, 2^{\prime}-\mathrm{H}\right.$ minor), $2.63\left(\mathrm{~m}, 1 \mathrm{H}, 4^{\prime \prime \prime}-\mathrm{Hb}\right), 2.85(\mathrm{dt}$, $J=13.6 \mathrm{~Hz} / 7.2 \mathrm{~Hz}, 0.8 \mathrm{H}, \mathrm{SCHa}$ major), $2.94(\mathrm{dt}, J=13.9 \mathrm{~Hz} / 7.1 \mathrm{~Hz}$, $0.8 \mathrm{H}, \mathrm{SCH} b$ major), 3.08 (m, 0.4H, SCH 2 minor), $3.16\left(\mathrm{~m}, 0.8 \mathrm{H}, 1^{\prime}-\mathrm{H}\right.$ major), 3.44-3.52 (m, $\left.4 \mathrm{H}, \mathrm{OCH}_{2} \mathrm{CH}_{2} \mathrm{OH}\right), 3.76\left(\mathrm{t}, J=5.2 \mathrm{~Hz}, 1 \mathrm{H}, 5^{\prime \prime \prime}-\right.$ $H), 3.79\left(\mathrm{~m}, 0.2 \mathrm{H}, 1^{\prime}-\mathrm{H}\right.$ minor $), 3.94\left(\mathrm{~m}, 1 \mathrm{H}, 1^{\prime \prime \prime}-\mathrm{H}\right), 4.56(\mathrm{dt}$, $\left.J=8.7 \mathrm{~Hz} / 6.0 \mathrm{~Hz}, 1 \mathrm{H}, 2^{\prime \prime \prime}-H\right), 4.60\left(\mathrm{t}, J=4.9 \mathrm{~Hz}, 1 \mathrm{H}, \mathrm{OCH}_{2} \mathrm{CH}_{2} \mathrm{OH}\right)$, $4.96\left(\mathrm{q}, 9.1 \mathrm{~Hz}, 1 \mathrm{H}, 3^{\prime \prime \prime}-\mathrm{H}\right), 5.05\left(\mathrm{~d}, J=4.0 \mathrm{~Hz}, 1 \mathrm{H}, 1^{\prime \prime \prime}-\mathrm{OH}\right), 5.12(\mathrm{~m}$, $1 \mathrm{H}, 2^{\prime \prime \prime}-\mathrm{OH}$ ), 7.04 (bs, $0.2 \mathrm{H}, 6^{\prime \prime}-\mathrm{H}$ minor), 7.07 (bs, $0.8 \mathrm{H}, 6^{\prime \prime}-\mathrm{H}$ major), 7.23-7.37 (m, 2H, $\left.2^{\prime \prime}-H / 5^{\prime \prime}-H\right), 8.94$ (d, $J=4.6 \mathrm{~Hz}, 0.2 \mathrm{H}, \mathrm{NH}$ minor), $9.36\left(\mathrm{~d}, J=3.6 \mathrm{~Hz}, 0.8 \mathrm{H}, \mathrm{NH}\right.$ major). ${ }^{13} \mathrm{C}$ NMR (DMSO- $\left.d_{6}\right) \delta 13.0,15.0$,
$22.3,24.0,32.3,33.2,34.1,60.3,60.5,70.8,73.7,74.3,81.8,114.8$, $117.0,122.8,123.2,139.3,146.8,148.4,148.7,149.4,150.3,150.8$, 154.0, 169.2. Anal. $\left(\mathrm{C}_{23} \mathrm{H}_{28} \mathrm{~F}_{2} \mathrm{~N}_{6} \mathrm{O}_{4} \mathrm{~S}\right)$ theoretical: $\mathrm{C}, 52.86 ; \mathrm{H}, 5.40 ; \mathrm{N}$, 16.08; S, 6.13. Found: C, 52.75; H, 5.75; N, 16.06, S, 6.26.

\subsection{0. (1S,2R,3S,4R)-4-(7-amino-5-((2-hydroxypropyl)thio)-3H- [1,2,3]triazolo[4,5-d]pyrimidin-3-yl)cyclopentane-1,2,3-triol (7n)}

To a solution of $\mathbf{1 6 h}(0.15 \mathrm{~g}, 0.39 \mathrm{mmol})$ in $\mathrm{MeOH}(3 \mathrm{~mL})$, was added $\mathrm{HCl} 12 \mathrm{~N}(1 \mathrm{~mL})$. After $30 \mathrm{~min}$ at room temperature, methanol was evaporated under reduced pressure and the resulting residue was suspended in water $(20 \mathrm{~mL})$, neutralized with $\mathrm{NaOH}$ $10 \%$ and extracted with dichloromethane $(3 \times 20 \mathrm{~mL})$. The combined organic layers were dried over $\mathrm{MgSO}_{4}$, concentrated under reduced pressure and purified by column chromatography on silica gel using ethyl acetate/methanol gradient to give $\mathbf{7 n}$ as a white solid (0.07 g, 52\% yield, m.p.: $177-180{ }^{\circ} \mathrm{C}$ ). ${ }^{1} \mathrm{H}$ NMR (DMSO- $\left.d_{6}\right) \delta 1.16$ (d, $\left.J=5.7 \mathrm{~Hz}, 3 \mathrm{H}, \mathrm{CH}_{3}\right), 1.94\left(\mathrm{ddt}, J=13.2 \mathrm{~Hz} / 8.5 \mathrm{~Hz} / 4.1 \mathrm{~Hz}, 1 \mathrm{H}, 5^{\prime}-\mathrm{Ha}\right)$, $2.57\left(\mathrm{~m}, 1 \mathrm{H}, 5^{\prime}-\mathrm{Hb}\right), 3.17(\mathrm{~m}, 2 \mathrm{H}, \mathrm{SCH} 2), 3.78\left(\mathrm{bs}, 1 \mathrm{H}, 2^{\prime}-\mathrm{H}\right), 3.87(\mathrm{~m}$, $\left.1 \mathrm{H}, \mathrm{SCH}_{2} \mathrm{CHOH}\right), 3.93\left(\mathrm{~m}, 1 \mathrm{H}, 1^{\prime}-\mathrm{H}\right), 4.67\left(\mathrm{~m}, 1 \mathrm{H}, 3^{\prime}-\mathrm{H}\right), 4.85(\mathrm{~d}$, $\left.J=4.6 \mathrm{~Hz}, 0.5 \mathrm{H}, S-\mathrm{SCH}_{2} \mathrm{CHOH}\right), 4.87(\mathrm{~d}, J=4.8 \mathrm{~Hz}, 0.5 \mathrm{H}$, $\left.R-\mathrm{SCH}_{2} \mathrm{CHOH}\right), 4.95-4.98\left(\mathrm{~m}, 2 \mathrm{H}, 2^{\prime}-\mathrm{OH} / 4^{\prime}-\mathrm{H}\right), 5.04$ (bs, $\left.1 \mathrm{H}, 3^{\prime}-\mathrm{OH}\right)$, $5.17\left(\mathrm{~d}, J=3.3 \mathrm{~Hz}, 1 \mathrm{H}, 1^{\prime}-\mathrm{OH}\right), 8.07(\mathrm{~s}, 1 \mathrm{H}, \mathrm{NHa}), 8.42(\mathrm{~s}, 1 \mathrm{H}, \mathrm{NHb}) .{ }^{13} \mathrm{C}$ NMR (DMSO-d $\left.d_{6}\right) \delta 22.6,35.7,38.8,61.1,65.4,73.1,74.3,76.7,122.8$, 149.5, 155.1, 169.1. Anal. $\left(\mathrm{C}_{12} \mathrm{H}_{18} \mathrm{~N}_{6} \mathrm{O}_{4} \mathrm{~S}\right)$ theoretical: C, 42.10; $\mathrm{H}, 5.30$; N, 24.55; S, 9.37. Found: C, 42.17; H, 5.56; N, 24.47, S, 9.39.

\subsection{1. (1S,2R,3S,4R)-4-(7-amino-5-((3-hydroxypropyl)thio)-3H- [1,2,3]triazolo[4,5-d]pyrimidin-3-yl)cyclopentane-1,2,3-triol (7o)}

To a solution of $\mathbf{1 6} \mathbf{i}(0.15 \mathrm{~g}, 0.33 \mathrm{mmol})$ in $\mathrm{MeOH}(3 \mathrm{~mL})$, was added $\mathrm{HCl} 12 \mathrm{~N}(1 \mathrm{~mL})$. After $30 \mathrm{~min}$ at room temperature, methanol was evaporated under reduced pressure and the resulting residue was suspended in water $(20 \mathrm{~mL})$, neutralized with $\mathrm{NaOH}$ $10 \%$ and extracted with dichloromethane $(3 \times 20 \mathrm{~mL})$. The combined organic layers were dried over $\mathrm{MgSO}_{4}$, concentrated under reduced pressure and purified by column chromatography on silica gel using ethyl acetate/methanol gradient to give $\mathbf{7 0}$ as a white solid (0.08 g, 59\% yield, m.p.: $\left.185-187^{\circ} \mathrm{C}\right) .{ }^{1} \mathrm{H}$ NMR (DMSO- $\left.d_{6}\right) \delta 1.82(\mathrm{~m}$, $\left.2 \mathrm{H}, \mathrm{SCH}_{2} \mathrm{CH}_{2} \mathrm{CH}_{2} \mathrm{OH}\right), 1.94\left(\mathrm{~m}, 1 \mathrm{H}, 5^{\prime}-\mathrm{Ha}\right), 2.59\left(\mathrm{~m}, 1 \mathrm{H}, 5^{\prime}-\mathrm{Hb}\right), 3.14$ (m, $\left.2 \mathrm{H}, \mathrm{SCH}_{2} \mathrm{CH}_{2} \mathrm{CH}_{2} \mathrm{OH}\right), 3.52\left(\mathrm{SCH}_{2} \mathrm{CH}_{2} \mathrm{CH}_{2} \mathrm{OH}\right), 3.79\left(\mathrm{~m}, 1 \mathrm{H}, 2^{\prime}-\mathrm{H}\right)$, $3.94\left(\mathrm{~m}, 1 \mathrm{H}, 1^{\prime}-\mathrm{H}\right), 4.52\left(\mathrm{~m}, 1 \mathrm{H}, \mathrm{SCH}_{2} \mathrm{CH}_{2} \mathrm{CH}_{2} \mathrm{OH}\right), 4.67\left(\mathrm{~m}, 1 \mathrm{H}, 3^{\prime}-\mathrm{H}\right)$, $4.91\left(\mathrm{~m}, 1 \mathrm{H}, 2^{\prime}-\mathrm{OH}\right), 4.96\left(\mathrm{~d}, J=8.4 \mathrm{~Hz}, 1 \mathrm{H}, 4^{\prime}-\mathrm{H}\right), 5.02\left(\mathrm{~m}, 1 \mathrm{H}, 3^{\prime}-\right.$ $\mathrm{OH}), 5.10\left(\mathrm{~m}, 1 \mathrm{H}, 1^{\prime}-\mathrm{OH}\right), 8.04(\mathrm{~s}, 1 \mathrm{H}, \mathrm{NHa}), 8.40(\mathrm{~s}, 1 \mathrm{H}, \mathrm{NHb}) .{ }^{13} \mathrm{C}$ NMR (DMSO-d $\left.d_{6}\right) \delta 27.1,32.2,35.9,59.4,61.1,73.1,74.4,76.7,122.8$, 149.6, 155.1, 169.0. Anal. $\left(\mathrm{C}_{12} \mathrm{H}_{18} \mathrm{~N}_{6} \mathrm{O}_{4} \mathrm{~S}\right)$ theoretical: $\mathrm{C}, 42.10 ; \mathrm{H}$, 5.30; N, 24.55; S, 9.37. Found: C, 42.46; H, 5.61; N, 24.36, S, 9.60.

\subsection{2. (1S,2R,3S,4R)-4-(7-(((1R,2S)-2-(3,4-difluorophenyl)} cyclopropyl)amino $)-5-((3-h y d r o x y p r o p y l)$ thio $)-3 H-[1,2,3]$ triazolo [4,5-d]pyrimidin-3-yl)cyclopentane-1,2,3-triol (7p)

To a solution of $\mathbf{1 6 j}(0.15 \mathrm{~g}, 0.28 \mathrm{mmol})$ in $\mathrm{MeOH}(3 \mathrm{~mL})$, was added $\mathrm{HCl} 12 \mathrm{~N}(1 \mathrm{~mL})$. After $30 \mathrm{~min}$ at room temperature, methanol was evaporated under reduced pressure and the resulting residue was suspended in water $(20 \mathrm{~mL})$, neutralized with $\mathrm{NaOH}$ $10 \%$ and extracted with dichloromethane $(3 \times 20 \mathrm{~mL})$. The combined organic layers were dried over $\mathrm{MgSO}_{4}$, concentrated under reduced pressure and purified by column chromatography on silica gel using ethyl acetate/methanol gradient to give $\mathbf{7 p}$ as a white solid (0.11 g, 79\% yield, m.p.: $147-149{ }^{\circ} \mathrm{C}$ ). ${ }^{1} \mathrm{H}$ NMR (DMSO- $\left.d_{6}\right) \delta 1.37$ (q, $J=6.4 \mathrm{~Hz}, 0.8 \mathrm{H}, 3^{\prime}-\mathrm{Ha}$ major), 1.40 (q, $J=6.7 \mathrm{~Hz}, 0.2 \mathrm{H}, 3^{\prime}-\mathrm{Ha}$ minor $)$, $1.46\left(\mathrm{~m}, 0.2 \mathrm{H}, 3^{\prime}-\mathrm{Hb}\right.$ minor), 1.52 (dt, $J=10.0 \mathrm{~Hz} / 5.3 \mathrm{~Hz}, 0.8 \mathrm{H}, 3^{\prime}-\mathrm{Hb}$ major), 1.66 (p, $J=6.6 \mathrm{~Hz}, 1.6 \mathrm{H}, \mathrm{SCH}_{2} \mathrm{CH}_{2} \mathrm{CH}_{2} \mathrm{OH}$ major), 1.81 (p, $J=6.3 \mathrm{~Hz}, 0.4 \mathrm{H}, \mathrm{SCH}_{2} \mathrm{CH}_{2} \mathrm{CH}_{2} \mathrm{OH}$ minor), 1.91 (ddd, $J=13.3 \mathrm{~Hz}$ ) 
$\left.9.0 \mathrm{~Hz} / 4.2 \mathrm{~Hz}, 1 \mathrm{H}, 5^{\prime \prime \prime}-\mathrm{Ha}\right), 2.14$ (m, 0.8H, 2'-H major), 2.25 (m, 0.2H, 2'-H minor), 2.59 (m, 1H, 5'"'-Hb), 2.95 (dt, $J=13.7 \mathrm{~Hz} / 7.1 \mathrm{~Hz}, 0.8 \mathrm{H}$, $\mathrm{SCHa}$ major), 3.02 (dt, $J=13.8 \mathrm{~Hz} / 7.0 \mathrm{~Hz}, 0.8 \mathrm{H}, \mathrm{SCH} b$ major), $3.16(\mathrm{~m}$, $1.2 \mathrm{H}$, SCH 2 minor $/ 1^{\prime}-H$ major), 3.38 (q, $J=5.9 \mathrm{~Hz}, 1.6 \mathrm{H}$, $\mathrm{SCH}_{2} \mathrm{CH}_{2} \mathrm{CH}_{2} \mathrm{OH}$ major), 3.52 (q, $J=5.5 \mathrm{~Hz}, 0.4 \mathrm{H}, \mathrm{SCH}_{2} \mathrm{CH}_{2} \mathrm{CH}_{2} \mathrm{OH}$ minor), $3.78\left(\mathrm{~m}, 1.2 \mathrm{H}, 1^{\prime}-H\right.$ minor $\left./ 2^{\prime \prime \prime}-H\right), 3.93\left(\mathrm{~m}, 1 \mathrm{H}, 1^{\prime \prime \prime}-H\right), 4.47(\mathrm{t}$, $J=5.1 \mathrm{~Hz}, 0.8 \mathrm{H}, \mathrm{SCH}_{2} \mathrm{CH}_{2} \mathrm{CH}_{2} \mathrm{OH}$ major), $4.53(\mathrm{t}, J=4.8 \mathrm{~Hz}, 0.2 \mathrm{H}$, $\mathrm{SCH}_{2} \mathrm{CH}_{2} \mathrm{CH}_{2} \mathrm{OH}$ minor), $4.65\left(\mathrm{~m}, 1 \mathrm{H}, 3^{\prime \prime \prime}-H\right), 4.92(\mathrm{~d}, J=3.8 \mathrm{~Hz}, 1 \mathrm{H}$, $\left.2^{\prime \prime \prime}-\mathrm{OH}\right), 4.95\left(\mathrm{q}, J=9.0 \mathrm{~Hz}, 1 \mathrm{H}, 4^{\prime \prime \prime}-H\right), 5.00\left(\mathrm{~d}, J=6.5 \mathrm{~Hz}, 0.2 \mathrm{H}, 3^{\prime \prime \prime}-\right.$ $\mathrm{OH}$ minor), 5.02 (d, $J=6.4 \mathrm{~Hz}, 0.8 \mathrm{H}, 3^{\prime \prime \prime}-\mathrm{OH}$ major), 5.09 (d, $J=3.8 \mathrm{~Hz}, 0.2 \mathrm{H}, 1^{\prime \prime \prime}-\mathrm{OH}$ minor), 5.11 (d, $J=4.0 \mathrm{~Hz}, 0.8 \mathrm{H}, 1^{\prime \prime \prime}-\mathrm{OH}$ major), 7.03 (bs, $0.2 \mathrm{H}, 6^{\prime \prime}-\mathrm{H}$ minor), 7.07 (bs, 0.8H, 6"- $\mathrm{H}$ major), 7.24-7.35 (m, 2H, 2" $\left.-H / 5^{\prime \prime}-H\right), 8.94$ (d, $J=4.5 \mathrm{~Hz}, 0.2 \mathrm{H}, \mathrm{NH}$ minor), $9.34\left(\mathrm{~d}, J=3.7 \mathrm{~Hz}, 0.8 \mathrm{H}, \mathrm{NH}\right.$ major). ${ }^{13} \mathrm{C}$ NMR (DMSO- $\left.d_{6}\right) \delta 15.1,23.9$, 27.2, 32.2, 33.9, 36.0, 59.2, 61.0, 73.2, 74.5, 76.7, 114.8, 117.0, 122.8, $123.1,139.2,147.0,148.4,149.3,150.0,153.9,169.1$. Anal. $\left(\mathrm{C}_{21} \mathrm{H}_{24} \mathrm{~F}_{2} \mathrm{~N}_{6} \mathrm{O}_{4} \mathrm{~S}\right)$ theoretical: $\mathrm{C}, 51.00 ; \mathrm{H}, 4.89 ; \mathrm{N}, 16.99 ; \mathrm{S}, 6.48$. Found: C, 50.68; H, 4.93; N, 16.56, S, 6.34 .

4.73. (1S,2R,3S,4R)-4-(7-(((1R,2S)-2-(3,4-difluorophenyl) cyclopropyl)amino)-5-(((R)-2-hydroxypropyl)thio)-3H-[1,2,3] triazolo[4,5-d]pyrimidin-3-yl)cyclopentane-1,2,3-triol (7q)

To a solution of 16k $(0.15 \mathrm{~g}, 0.28 \mathrm{mmol})$ in $\mathrm{MeOH}(3 \mathrm{~mL})$, was added $\mathrm{HCl} 12 \mathrm{~N}(1 \mathrm{~mL})$. After $30 \mathrm{~min}$ at room temperature, methanol was evaporated under reduced pressure and the resulting residue was suspended in water $(20 \mathrm{~mL})$, neutralized with $\mathrm{NaOH}$ $10 \%$ and extracted with dichloromethane $(3 \times 20 \mathrm{~mL})$. The combined organic layers were dried over $\mathrm{MgSO}_{4}$, concentrated under reduced pressure and purified by column chromatography on silica gel using ethyl acetate/methanol gradient to give $\mathbf{7 q}$ as a white solid (0.09 g, 65\% yield, m.p.: $90{ }^{\circ} \mathrm{C}$ dec.). ${ }^{1} \mathrm{H}$ NMR (DMSO- $\left.d_{6}\right) \delta 1.00$ (d, $J=6.0 \mathrm{~Hz}, 2.4 \mathrm{H}, \mathrm{CH}_{3}$ major), $1.17\left(\mathrm{~d}, J=6.9 \mathrm{~Hz}, 0.6 \mathrm{H}, \mathrm{CH}_{3}\right.$ minor $)$, $1.38\left(\mathrm{~m}, 1 \mathrm{H}, 3^{\prime}-\mathrm{Ha}\right), 1.46\left(\mathrm{~m}, 0.2 \mathrm{H}, 3^{\prime}-\mathrm{Hb}\right.$ minor), $1.53\left(\mathrm{~m}, 0.8 \mathrm{H}, 3^{\prime}-\mathrm{Hb}\right.$ major), 1.92 (ddd, $J=13.3 \mathrm{~Hz} / 8.9 \mathrm{~Hz} / 4.1 \mathrm{~Hz}, 1 \mathrm{H}, 5^{\prime \prime \prime}-\mathrm{Ha}$ ), 2.13 (m, $0.8 \mathrm{H}, 2^{\prime}-H$ major), 2.25 ( $\mathrm{m}, 0.2 \mathrm{H}, 2^{\prime}-H$ minor), $2.60\left(\mathrm{~m}, 1 \mathrm{H}, 5^{\prime \prime \prime}-H b\right)$, 2.89 (dd, $J=13.4 \mathrm{~Hz} / 6.8 \mathrm{~Hz}, 0.8 \mathrm{H}, \mathrm{SCHa}$ major), 3.14 (m, 1.8H, SCHa minor/SCHb major/1'- $H$ major), 3.21 (dd, $J=13.5 \mathrm{~Hz} / 5.2 \mathrm{~Hz}, 0.2 \mathrm{H}$, $\mathrm{SCHb}$ minor), $3.72-3.78$ ( $\mathrm{m}, 2 \mathrm{H}, \mathrm{SCH}_{2} \mathrm{CHOH}$ major $/ 1^{\prime}-\mathrm{H}$ minor $/ 2^{\prime \prime \prime}-$ $H), 3.87\left(\mathrm{~m}, 0.2 \mathrm{H}, \mathrm{SCH}_{2} \mathrm{CHOH}\right.$ minor), $3.93\left(\mathrm{~m}, 1 \mathrm{H}, 1^{\prime \prime \prime}-\mathrm{H}\right), 4.66(\mathrm{q}$, $\left.J=6.3 \mathrm{~Hz}, 1 \mathrm{H}, 3^{\prime \prime \prime}-H\right), 4.80$ (d, $J=4.8 \mathrm{~Hz}, 0.8 \mathrm{H}, \mathrm{SCH}_{2} \mathrm{CHOH}$ major), $4.87\left(\mathrm{~d}, J=4.5 \mathrm{~Hz}, 0.2 \mathrm{H}, \mathrm{SCH}_{2} \mathrm{CHOH}\right.$ minor), $4.95(\mathrm{~d}, J=4.3 \mathrm{~Hz}, 1 \mathrm{H}$, $\left.2^{\prime \prime \prime}-\mathrm{OH}\right), 4.98\left(\mathrm{~m}, 1.2 \mathrm{H}, 4^{\prime \prime \prime}-\mathrm{H} / 3^{\prime \prime \prime}-\mathrm{OH}\right.$ minor), $5.04(\mathrm{~d}, J=6.4 \mathrm{~Hz}, 0.8 \mathrm{H}$, $3^{\prime \prime \prime}-\mathrm{OH}$ major), 5.17 (d, $J=3.7 \mathrm{~Hz}, 1 \mathrm{H}, 1^{\prime \prime \prime}-\mathrm{OH}$ ), 7.04 (bs, $0.2 \mathrm{H}, 6^{\prime \prime}-\mathrm{H}$ minor), 7.09 (bs, 0.8H, 6"-H major), 7.24-7.37 (m, 2H, 2" $-H / 5^{\prime \prime}-H$ ), $8.96(\mathrm{~d}, J=4.4 \mathrm{~Hz}, 0.2 \mathrm{H}, \mathrm{NH}$ minor), $9.36(\mathrm{~d}, J=3.9 \mathrm{~Hz}, 0.8 \mathrm{H}, \mathrm{NH}$ major). ${ }^{13} \mathrm{C}$ NMR (DMSO- $\left.d_{6}\right) \delta 15.0,22.4,24.0,33.9,35.9,39.6,61.1$, 65.2, 73.2, 74.5, 76.7, 114.9, 117.0, 122.9, 123.1, 139.2, 147.1, 148.4, 148.7, 149.2, 150.1, 153.9, 169.2. Anal. $\left(\mathrm{C}_{21} \mathrm{H}_{24} \mathrm{~F}_{2} \mathrm{~N}_{6} \mathrm{O}_{4} \mathrm{~S}\right)$ theoretical: C, 51.00; H, 4.89; N, 16.99; S, 6.48. Found: C, 50.77; H, 5.15; N, 16.64, S, 6.99 .

4.74. (1S,2R,3S,4R)-4-(7-(((1R,2S)-2-(3,4-difluorophenyl) cyclopropyl)amino)-5-(((S)-2-hydroxypropyl)thio)-3H-[1,2,3] triazolo[4,5-d]pyrimidin-3-yl)cyclopentane-1,2,3-triol (7r)

To a solution of $\mathbf{1 6 1}(0.15 \mathrm{~g}, 0.28 \mathrm{mmol})$ in $\mathrm{MeOH}(3 \mathrm{~mL})$, was added $\mathrm{HCl} 12 \mathrm{~N}(1 \mathrm{~mL})$. After $30 \mathrm{~min}$ at room temperature, methanol was evaporated under reduced pressure and the resulting residue was suspended in water $(20 \mathrm{~mL})$, neutralized with $\mathrm{NaOH}$ $10 \%$ and extracted with dichloromethane $(3 \times 20 \mathrm{~mL})$. The combined organic layers were dried over $\mathrm{MgSO}_{4}$, concentrated under reduced pressure and purified by column chromatography on silica gel using ethyl acetate/methanol gradient to give $7 \mathbf{r}$ as a white solid (0.10 g, 72\% yield, m.p.: $90{ }^{\circ} \mathrm{C}$ dec.). ${ }^{1} \mathrm{H}$ NMR (DMSO-d 6 ) $\delta 1.02$ (d,
$J=6.1 \mathrm{~Hz}, 2.4 \mathrm{H}, \mathrm{CH}_{3}$ major), 1.17 (d, $J=6.0 \mathrm{~Hz}, 0.6 \mathrm{H}, \mathrm{CH}_{3}$ minor), 1.38 ( $\left.\mathrm{m}, 1 \mathrm{H}, 3^{\prime}-\mathrm{Ha}\right), 1.46\left(\mathrm{~m}, 0.2 \mathrm{H}, 3^{\prime}-\mathrm{Hb}\right.$ minor), $1.52\left(\mathrm{~m}, 0.8 \mathrm{H}, 3^{\prime}-\mathrm{Hb}\right.$ major), $1.92\left(\mathrm{~m}, 1 \mathrm{H}, 5^{\prime \prime \prime}-\mathrm{Ha}\right), 2.15\left(\mathrm{~m}, 0.8 \mathrm{H}, 2^{\prime}-\mathrm{H}\right.$ major), $2.25(\mathrm{~m}$, $0.2 \mathrm{H}, 2^{\prime}-\mathrm{H}$ minor), $2.60\left(\mathrm{~m}, 1 \mathrm{H}, 5^{\prime \prime \prime}-\mathrm{Hb}\right), 3.06$ ( $\mathrm{m}, 1.6 \mathrm{H}, \mathrm{SCH} 2$ major), $3.18\left(\mathrm{~m}, 1.2 \mathrm{H}, 1^{\prime}-\mathrm{H}\right.$ major/ $\mathrm{SCH} 2$ minor $), 3.73-3.78(\mathrm{~m}, 2 \mathrm{H}$, $\mathrm{SCH}_{2} \mathrm{CHOH}$ major $/ 1^{\prime}-\mathrm{H}$ minor $\left./ 2^{\prime \prime \prime}-\mathrm{H}\right), 3.88\left(\mathrm{~m}, 0.2 \mathrm{H}, \mathrm{SCH}_{2} \mathrm{CHOH}\right.$ minor), $3.93\left(\mathrm{~m}, 1 \mathrm{H}, 1^{\prime \prime \prime}-\mathrm{H}\right), 4.66\left(\mathrm{~m}, 1 \mathrm{H}, 3^{\prime \prime \prime}-\mathrm{H}\right), 4.76(\mathrm{~d}, J=4.7 \mathrm{~Hz}$, $0.8 \mathrm{H}, \mathrm{SCH}_{2} \mathrm{CHOH}$ major), $4.84\left(\mathrm{~d}, 4.5 \mathrm{~Hz}, 0.2 \mathrm{H}, \mathrm{SCH}_{2} \mathrm{CHOH}\right.$ minor), $4.93\left(\mathrm{~d}, J=3.8 \mathrm{~Hz}, 1 \mathrm{H}, 2^{\prime \prime \prime}-\mathrm{OH}\right), 4.96\left(\mathrm{q}, J=9.0 \mathrm{~Hz}, 1 \mathrm{H}, 4^{\prime \prime \prime}-\mathrm{H}\right), 4.99(\mathrm{~d}$, $J=6.5 \mathrm{~Hz}, 0.2 \mathrm{H}, 3^{\prime \prime \prime}-\mathrm{OH}$ minor), $5.02\left(\mathrm{~d}, J=6.4 \mathrm{~Hz}, 0.8 \mathrm{H}, 3^{\prime \prime \prime}-\mathrm{OH}\right.$ major), $5.16\left(\mathrm{~d}, J=4.0 \mathrm{~Hz}, 1 \mathrm{H}, 1^{\prime \prime \prime}-\mathrm{OH}\right), 7.04$ (bs, $0.2 \mathrm{H}, 6^{\prime \prime}-\mathrm{H}$ minor), 7.09 (bs, 0.8H, 6" $-H$ major), 7.24-7.35 (m, 2H, 2" $-H / 5^{\prime \prime}-H$ ), 8.94 (d, $J=4.4 \mathrm{~Hz}, 0.2 \mathrm{H}, \mathrm{NH}$ minor), 9.33 (d, $J=3.9 \mathrm{~Hz}, 0.8 \mathrm{H}, \mathrm{NH}$ major) ${ }^{13} \mathrm{C}$ NMR (DMSO- $\left.d_{6}\right) \delta 15.1,22.4,23.9,33.8,36.0,39.1,61.0,65.2,73.2$, 74.5, 76.8, 114.9, 117.0, 122.9, 123.1, 139.2, 147.1, 148.7, 149.2, 153.8, 169.3. Anal. $\left(\mathrm{C}_{21} \mathrm{H}_{24} \mathrm{~F}_{2} \mathrm{~N}_{6} \mathrm{O}_{4} \mathrm{~S} . \mathrm{H}_{2} \mathrm{O}\right)$ theoretical: $\mathrm{C}, 49.21 ; \mathrm{H}, 5.11 ; \mathrm{N}$, 16.40; S, 6.26. Found: C, 49.56; H, 5.05; N, 16.18, S, 5.99.

4.75. (1S,2S,3R,5S)-3-(7-(((1R,2S)-2-(3,4-difluorophenyl) cyclopropyl)amino)-5-((2-hydroxypropyl)thio)-3H-[1,2,3]triazolo [4,5-d]pyrimidin-3-yl)-5-(2-hydroxyethoxy)cyclopentane-1,2-diol (7s)

To a solution of $16 \mathrm{~m}(0.15 \mathrm{~g}, 0.26 \mathrm{mmol})$ in $\mathrm{MeOH}(3 \mathrm{~mL})$, was added $\mathrm{HCl} 12 \mathrm{~N}(1 \mathrm{~mL})$. After $30 \mathrm{~min}$ at room temperature, methanol was evaporated under reduced pressure and the resulting residue was suspended in water $(20 \mathrm{~mL})$, neutralized with $\mathrm{NaOH}$ $10 \%$ and extracted with dichloromethane $(3 \times 20 \mathrm{~mL})$. The combined organic layers were dried over $\mathrm{MgSO}_{4}$, concentrated under reduced pressure and purified by column chromatography on silica gel using ethyl acetate/methanol gradient to give $7 \mathbf{s}$ as a white solid (0.12 g, 86\% yield, m.p.: $\left.148-153^{\circ} \mathrm{C}\right) .{ }^{1} \mathrm{H}$ NMR (DMSO- $\left.d_{6}\right) \delta 1.00$ (d, $J=6.2 \mathrm{~Hz}, 1.2 \mathrm{H}, \mathrm{CH}_{3}$ major $R$ or $\left.S\right), 1.02\left(\mathrm{~d}, J=6.2 \mathrm{~Hz}, 1.2 \mathrm{H}, \mathrm{CH}_{3}\right.$ major $R$ or $S), 1.17\left(\mathrm{~d}, J=7.1 \mathrm{~Hz}, 0.6 \mathrm{H}, \mathrm{CH}_{3}\right.$ minor), $1.38\left(\mathrm{~m}, 1 \mathrm{H}, 3^{\prime}-\mathrm{Ha}\right), 1.47$ ( $\mathrm{m}, 0.2 \mathrm{H}, 3^{\prime}-\mathrm{Hb}$ minor), 1.53 ( $\mathrm{m}, 0.8 \mathrm{H}, 3^{\prime}-\mathrm{Hb}$ major), 2.02 (ddt, $\left.J=14.0 \mathrm{~Hz} / 9.3 \mathrm{~Hz} / 4.4 \mathrm{~Hz}, 1 \mathrm{H}, 4^{\prime \prime \prime}-\mathrm{Ha}\right), 2.14$ (m, 0.8H, 2'-H major), $2.25\left(\mathrm{~m}, 0.2 \mathrm{H}, 2^{\prime}-H\right.$ minor), $2.64\left(\mathrm{~m}, 1 \mathrm{H}, 4^{\prime \prime \prime}-\mathrm{Hb}\right), 2.86$ (dd, $J=13.4 \mathrm{~Hz} / 7.0 \mathrm{~Hz}, 0.4 \mathrm{H}, \mathrm{SCHa}$ major $R$ or $S), 3.05(\mathrm{~d}, J=5.8 \mathrm{~Hz}, 1 \mathrm{H}$, $\mathrm{SCHb}$ major), 3.13-3.19 (m, 1.6H, SCHa major $R$ or $S$, SCHa minor, $\mathrm{SCH} b$ minor, $\left.1^{\prime}-\mathrm{H}\right), 3.46-3.52\left(\mathrm{~m}, 4 \mathrm{H}, \mathrm{OCH}_{2} \mathrm{CH}_{2} \mathrm{OH}\right), 3.74(\mathrm{~m}, 1.8 \mathrm{H}$, $\mathrm{SCH}_{2} \mathrm{CHOH}$ major $\left./ 5^{\prime \prime \prime}-\mathrm{H}\right), 3.88\left(\mathrm{~m}, 0.2 \mathrm{H}, \mathrm{SCH}_{2} \mathrm{CHOH}\right.$ minor), 3.95 (m, $\left.1 \mathrm{H}, 1^{\prime \prime \prime}-\mathrm{H}\right), 4.56\left(\mathrm{~m}, 1 \mathrm{H}, 2^{\prime \prime \prime}-H\right), 4.61\left(\mathrm{t}, J=4.9 \mathrm{~Hz}, \mathrm{OCH}_{2} \mathrm{CH}_{2} \mathrm{OH}\right), 4.78$ (m, $0.8 \mathrm{H}, \mathrm{SCH}_{2} \mathrm{CHOH}$ major), $4.86\left(\mathrm{~m}, 0.8 \mathrm{H}, \mathrm{SCH}_{2} \mathrm{CHOH}\right.$ minor), 4.96 (q, $\left.J=8.7 \mathrm{~Hz}, 1 \mathrm{H}, 3^{\prime \prime \prime}-H\right), 5.06$ (d, $\left.J=3.8 \mathrm{~Hz}, 1 \mathrm{H}, 1^{\prime \prime \prime}-\mathrm{OH}\right), 5.09-5.12$ (m, 1H, 2"'-OH), 7.03 (m, 0.2H, 6"-H minor), $7.09\left(\mathrm{~m}, 0.8 \mathrm{H}, 6^{\prime \prime}-H\right)$, $7.24-7.36\left(\mathrm{~m}, 2 \mathrm{H}, 2^{\prime \prime}-\mathrm{H} / 5^{\prime \prime}-H\right), 8.96$ (d, $J=4.5 \mathrm{~Hz}, 0.2 \mathrm{H}, \mathrm{NH}$ minor), 9.35 ( $\mathrm{m}, 0.8 \mathrm{H}, \mathrm{NH}$ major). ${ }^{13} \mathrm{C}$ NMR (DMSO- $\left.d_{6}\right) \delta 15.1,22.5,24.0$, 33.3, 33.9, 35.7, 39.4, 60.3, 60.5, 65.2, 70.9, 73.7, 74.4, 81.8, 114.9, 117.0, 122.9, 123.1, 139.2, 147.1, 148.5, 148.7, 149.4, 150.1, 153.9, 169.4. Anal. $\left(\mathrm{C}_{23} \mathrm{H}_{28} \mathrm{~F}_{2} \mathrm{~N}_{6} \mathrm{O}_{5} \mathrm{~S}\right)$ theoretical: $\mathrm{C}, 51.29 ; \mathrm{H}, 5.24 ; \mathrm{N}, 15.60 ; \mathrm{S}$, 5.95. Found: C, 51.29; H, 5.49; N, 15.51, S, 5.85.

4.76. (1S,2S,3R,5S)-3-(7-(((1R,2S)-2-(3,4-difluorophenyl) cyclopropyl)amino)-5-((3-hydroxypropyl)thio)-3H-[1,2,3]triazolo [4,5-d]pyrimidin-3-yl)-5-(2-hydroxyethoxy)cyclopentane-1,2-diol (7t)

To a solution of $16 \mathrm{n}(0.15 \mathrm{~g}, 0.26 \mathrm{mmol})$ in $\mathrm{MeOH}(3 \mathrm{~mL})$, was added $\mathrm{HCl} 12 \mathrm{~N}$ (1 mL). After $30 \mathrm{~min}$ at room temperature, methanol was evaporated under reduced pressure and the resulting residue was suspended in water $(20 \mathrm{~mL})$, neutralized with $\mathrm{NaOH}$ $10 \%$ and extracted with dichloromethane $(3 \times 20 \mathrm{~mL})$. The combined organic layers were dried over $\mathrm{MgSO}_{4}$, concentrated under reduced pressure and purified by column chromatography on silica gel using ethyl acetate/methanol gradient to give $\mathbf{7 t}$ as a white solid 
(0.10 g, 72\% yield, m.p.: $135-137.5^{\circ} \mathrm{C}$ ). ${ }^{1} \mathrm{H}$ NMR (DMSO- $\left.d_{6}\right) \delta 1.38$ (q, $J=6.1 \mathrm{~Hz}, 0.8 \mathrm{H}, 3^{\prime}-\mathrm{Ha}$ major $), 1.40$ ( $\mathrm{m}, 0.2 \mathrm{H}, 3^{\prime}-\mathrm{Ha}$ minor), 1.46 (dt, $J=9.9 \mathrm{~Hz} / 5.3 \mathrm{~Hz}, 0.2 \mathrm{H}, 3^{\prime}-\mathrm{Hb}$ minor), 1.53 (dt, $J=11.0 \mathrm{~Hz} / 5.5 \mathrm{~Hz}$ $0.8 \mathrm{H}, 3^{\prime}-\mathrm{Hb}$ major), 1.66 ( $\mathrm{p}, J=6.7 \mathrm{~Hz}, 1.6 \mathrm{H}, \mathrm{SCH}_{2} \mathrm{CH}_{2} \mathrm{CH}_{2} \mathrm{OH}$ major), 1.81 (p, $J=6.6 \mathrm{~Hz}, 0.4 \mathrm{H}, \mathrm{SCH}_{2} \mathrm{CH}_{2} \mathrm{CH}_{2} \mathrm{OH}$ minor), 2.02 (ddd, $\left.J=14.0 \mathrm{~Hz} / 9.7 \mathrm{~Hz} / 5.0 \mathrm{~Hz}, 1 \mathrm{H}, 4^{\prime \prime \prime}-\mathrm{Ha}\right), 2.14$ (ddd, $J=9.5 \mathrm{~Hz} / 6.4 \mathrm{~Hz} /$ $3.3 \mathrm{~Hz}, 0.8 \mathrm{H}, 2^{\prime}-H$ major $), 2.25\left(\mathrm{~m}, 0.2 \mathrm{H}, 2^{\prime}-H\right.$ minor), $2.64(\mathrm{~m}, 1 \mathrm{H}$, $4^{\prime \prime \prime}-\mathrm{Hb}$ ), 2.93-3.03 (m, $1.6 \mathrm{H}, \mathrm{SCH}_{2} \mathrm{CH}_{2} \mathrm{CH}_{2} \mathrm{OH}$ major), 3.15-3.18 (m, $1.2 \mathrm{H}, \mathrm{SCH}_{2} \mathrm{CH}_{2} \mathrm{CH}_{2} \mathrm{OH}$ minor/1'- $\mathrm{H}$ major), $3.38(\mathrm{q}, J=6.1 \mathrm{~Hz}, 1.6 \mathrm{H}$, $\mathrm{SCH}_{2} \mathrm{CH}_{2} \mathrm{CH}_{2} \mathrm{OH}$ major), 3.46-3.53 (m, $4.4 \mathrm{H}, \mathrm{SCH}_{2} \mathrm{CH}_{2} \mathrm{CH}_{2} \mathrm{OH}$ minor/OCH $\left.\mathrm{OCH}_{2} \mathrm{OH}\right), 3.76\left(\mathrm{~m}, 1 \mathrm{H}, 5^{\prime \prime \prime}-\mathrm{H}\right), 3.79\left(\mathrm{~m}, 0.2 \mathrm{H}, 1^{\prime}-\mathrm{H}\right.$ minor $)$, $3.94\left(\mathrm{~m}, 1 \mathrm{H}, 1^{\prime \prime \prime}-\mathrm{H}\right), 4.47\left(\mathrm{t}, J=5.2 \mathrm{~Hz}, 0.8 \mathrm{H}, \mathrm{SCH}_{2} \mathrm{CH}_{2} \mathrm{CH}_{2} \mathrm{OH}\right.$ major), $4.55\left(\mathrm{~m}, 1.2 \mathrm{H}, \mathrm{SCH}_{2} \mathrm{CH}_{2} \mathrm{CH}_{2} \mathrm{OH}\right.$ minor $\left./ 2^{\prime \prime \prime}-\mathrm{H}\right), 4.61(\mathrm{t}, J=5.2 \mathrm{~Hz}, 1 \mathrm{H}$, $\left.\mathrm{OCH}_{2} \mathrm{CH}_{2} \mathrm{OH}\right), 4.96\left(\mathrm{q}, J=9.1 \mathrm{~Hz}, 1 \mathrm{H}, 3^{\prime \prime \prime}-\mathrm{H}\right), 5.05(\mathrm{~d}, J=4.1 \mathrm{~Hz}, 1 \mathrm{H}$, $\left.1^{\prime \prime \prime}-\mathrm{OH}\right), 5.09\left(\mathrm{~d}, J=6.5 \mathrm{~Hz}, 0.8 \mathrm{H}, 2^{\prime \prime \prime}-\mathrm{OH}\right.$ major), $5.12(\mathrm{~d}, J=6.4 \mathrm{~Hz}$ $0.2 \mathrm{H}, 2^{\prime \prime \prime}-\mathrm{OH}$ minor), $7.03\left(\mathrm{~m}, 0.2 \mathrm{H}, 6^{\prime \prime}-\mathrm{H}\right.$ minor), 7.07 ( $\mathrm{m}, 0.8 \mathrm{H}, 6^{\prime \prime}-\mathrm{H}$ major), 7.24-7.35 (m, 2H, 2" $\left.-H / 5^{\prime \prime}-H\right), 8.95$ (d, $J=4.8 \mathrm{~Hz}, 0.2 \mathrm{H}, \mathrm{NH}$ minor), 9.35 (d, $J=4.0 \mathrm{~Hz}, 0.8 \mathrm{H}, \mathrm{NH}$ major). ${ }^{13} \mathrm{C}$ NMR (DMSO-d $d_{6}$ ) $\delta$ 15.1, 23.9, 27.3, 32.1, 33.3, 33.9, 59.2, 60.3, 60.5, 70.9, 73.7, 74.4, 81.8, 115.0, 117.1, 122.8, 123.1, 139.2, 147.1, 148.4, 148.7, 149.4, 150.1, 153.9, 169.2. Anal. $\left(\mathrm{C}_{23} \mathrm{H}_{28} \mathrm{~F}_{2} \mathrm{~N}_{6} \mathrm{O}_{5} \mathrm{~S}\right)$ theoretical: $\mathrm{C}, 51.29 ; \mathrm{H}, 5.24 ; \mathrm{N}$, 15.60; S, 5.95. Found: C, 50.89; H, 5.29; N, 15.51, S, 5.99.

\subsection{Biological evaluation}

\subsubsection{Antiplatelet activity}

4.77.1.1. Platelet rich plasma (PRP) preparation. Venous blood was obtained from healthy volunteers (between 20 and 40 years old) who had not taken any drugs or medications for at least 10 days. Procedures were approved by the institutional review committee of the Universtisty of Liege (Comité d'éthique hospital-facultaire universitaire de liege). Blood samples were collected into citrate tubes (3.2\% citrate, Greiner-BioOne) and processed within $30 \mathrm{~min}$ after collection. Platelet-rich-plasma (PRP) was separated by centrifugation of anticoagulated whole blood at $100 \times \mathrm{g}$ for $15 \mathrm{~min}$ at room temperature. Platelet count was measured using a hematology analyser (Cell-dyn 3700, Abbott). Platelet poor plasma (PPP) was obtained by centrifugation of whole blood at $800 \times \mathrm{g}$ for $15 \mathrm{~min}$. The platelet count in PRP was then adjusted to $250000 / \mu \mathrm{l}$ by diluting PRP with autologous PPP.

4.77.1.2. Platelet aggregation. Platelet aggregation assays were performed with PRP under stirring (1200 rpm) at $37{ }^{\circ} \mathrm{C}$ on a Chrono-Log Lumi-aggregometer (Kordia). PRP was pre-incubated with ticagrelor or its analogues $(1.8 \mu \mathrm{M})$ for 10 min prior to stirring and addition of ADP $(10 \mu \mathrm{M})$. Platelet aggregation was monitored for $10 \mathrm{~min}$. The area under the curve (AUC) was recorded. Results were expressed as fold-inhibition vs. vehicle (1\% DMSO). Data are presented as mean \pm S.D. obtained from 4 to 21 healthy volunteers (age: 29 (3), median (IQR); 67\% males). AUC values recorded in the presence of test molecules were compared to the values obtained with the vehicle control (1\% DMSO) or with the reference molecule 1 (ticagrelor) by using the non-parametric Wilcoxon signed rank test for paired samples (Prism version 8.1.2). $P<0.05$ was considered significant.

\subsubsection{Antibacterial activity}

A single colony of S.aureus (MRSA, BAA-1556) grown on a Brain Heart Infusion Agar plate was resupended and cultured in Brain Heart infusion broth medium $(\mathrm{BHI})$ overnight $(\mathrm{O} / \mathrm{N})$ under aerobic conditions ( $37^{\circ} \mathrm{C}, 190 \mathrm{rpm}$ shaking). On the next day, the resulting inoculum was diluted 100 times in Mueller-Hinton broth (MHB) and incubated in aerobic conditions to reach a growth exponential phase. The resulting inoculum corresponding to about $5.10^{5}$ colony forming units ( $\mathrm{CFU}) / \mathrm{ml}$ was further incubated in the presence or in the absence of different concentrations of the tested molecules. The vehicle was $1 \%$ DMSO. The bacterial growth in culture media was evaluated by measuring the optical density (OD) at $600 \mathrm{~nm}\left(\mathrm{OD}_{600}\right)$ by use of a spectrophotometer. The Minimal Inhibitory Concentration (MIC) of each molecule tested was determined according to the EUCAST guidelines. The MIC represents the concentration at which there is no visible growth, i.e $\Delta \mathrm{OD}$ at $600 \mathrm{~nm}$ is equal to zero wherein $\triangle O D$ is the difference between the resulting OD in the presence of the molecule and the OD of the blank (blank is the medium with $1 \%$ DMSO).

\section{Author contributions}

The manuscript was written through contributions of all authors. All authors have given approval to the final version of the manuscript.

\section{Declaration of competing interest}

The authors declare that they have no known competing financial interests or personal relationships that could have appeared to influence the work reported in this paper.

\section{Acknowledgement}

C. Oury is Research Director at the National Funds for Scientific Research, Belgium (F.R.S.-FNRS). The technical assistance of S. Counerotte is gratefully acknowledged. This work was supported by a European Research Council (ERC)-Consolidator grant (PV- COAT 647197).

\section{Appendix A. Supplementary data}

Supplementary data to this article can be found online at https://doi.org/10.1016/j.ejmech.2020.112767.

\section{Abbreviations}

ADP adenosine diphosphate

DMSO dimethylsulfoxide

LSD1 lysine specific demethylase 1

MRSA methicillin-resistant Staphylococcus aureus

NMR nuclear magnetic resonance

TLC thin layer chromatography

TMS tetramethylsilane

\section{References}

[1] B. Springthorpe, A. Bailey, P. Barton, T.N. Birkinshaw, R.V. Bonnert, R.C. Brown, D. Chapman, J. Dixon, S.D. Guile, R.G. Humphries, S.F. Hunt, F. Ince, A.H. Ingall, I.P. Kirk, P.D. Leeson, P. Leff, R.J. Lewis, B.P. Martin, D.F. McGinnity, M.P. Mortimore, S.W. Paine, G. Pairaudeau, A. Patel, A.J. Rigby, R.J. Riley, B.J. Teobald, W. Tomlinson, P.J. Webborn, P.A. Willis, From ATP to AZD6140: the discovery of an orally active reversible P2Y12 receptor antagonist for the prevention of thrombosis, Bioorg. Med. Chem. Lett 17 (2007) 6013-6018.

[2] D.A. Lutz, K. Hoffmann, J. Straßburger, Y. Baqi, C.E. Müller, I. von Kügelgen, Competitive mode and site of interaction of ticagrelor at the human platelet P2Y12-receptor, J. Thromb. Haemostasis 12 (2014) 1898-1905.

[3] M. Cattaneo, New P2Y12 blockers, J. Thromb. Haemostasis 7 (Suppl 1) (2009) 262-265.

[4] P.A. Gurbel, K.P. Bliden, K. Butler, M.J. Antonino, C. Wei, R. Teng, L. Rasmussen, R.F. Storey, T. Nielsen, J.W. Eikelboom, G. Sabe-Affaki, S. Husted, D.J. Kereiakes, D. Henderson, D.V. Patel, U.S. Tantry, Response to ticagrelor in clopidogrel nonresponders and responders and effect of switching therapies: the RESPOND study, Circulation 121 (2010) 1188-1199.

[5] R. Teng, S. Oliver, M.A. Hayes, K. Butler, Absorption, distribution, metabolism, and excretion of ticagrelor in healthy subjects, Drug Metab. Dispos. 38 (2010) $1514-1521$.

[6] P. Lancellotti, L. Musumeci, N. Jacques, L. Servais, E. Goffin, B. Pirotte, C. Oury, Antibacterial activity of ticagrelor in conventional antiplatelet dosages against 
antibiotic-resistant Gram-positive bacteria, JAMA Cardiol 4 (2019) 596-599.

[7] H.S. Jeong, S.J. Hong, S.A. Cho, J.H. Kim, J.Y. Cho, S.H. Lee, H.J. Joo, J.H. Park, C.W. Yu, D.S. Lim, Comparison of ticagrelor versus prasugrel for inflammation, vascular Function, and circulating endothelial progenitor cells in diabetic patients with non-ST-segment elevation acute coronary syndrome requiring coronary stenting: a prospective, randomized, crossover trial, JACC Cardiovasc, Interv 10 (2017) 1646-1658.

[8] C.Z. Gao, Q.Q. Ma, J. Wu, R. Liu, F. Wang, J. Bai, X.J. Yang, Q. Fu, P. Wei, Comparison of the effects of ticagrelor and clopidogrel on inflammatory factors, vascular endothelium functions and short-term prognosis in patients with acute ST-segment elevation myocardial infarction undergoing emergency percutaneous coronary intervention: a pilot study, Cell. Physiol. Biochem. 48 (2018) 385-396.

[9] M.J. Kubisa, M.P. Jezewski, A. Gasecka, J.M. Siller-Matula, M. Postuła, Ticagrelor - toward more efficient platelet inhibition and beyond, Therapeut. Clin. Risk Manag. 14 (2018) 129-140.

[10] T.R Sexton, G. Zhang TE Macaulay, L A Callahan, R. Charnigo, O.A. Vsevolozhskaya, Z. Li, S. Smyth, Ticagrelor reduces thromboinflammatory markers in patients with pneumonia, JACC Basic Transl. Sci. 3 (2018) 435-449.

[11] M.F. Reiner, A. Akhmedov, S. Stivala, S. Keller, D.S. Gaul, N.R. Bonetti, G. Savarese, M. Glanzmann, C. Zhu, W. Ruf, Z Yang CM. Matter T.F. Lüscher, G.G. Camici, J.H. Beer, Ticagrelor, but not clopidogrel, reduces arterial thrombosis via endothelial tissue factor suppression, Cardiovasc. Res. 113 (2017) 61-69.

[12] H. Zhang J. Liu, L. Zhang, L. Kong, H. Yao, H. Sun, Synthesis and biological evaluation of ticagrelor derivatives as novel antiplatelet agents, Bioorg. Med. Chem. Lett 22 (2012) 3598-3602.

[13] Y. Wang, H. Yan, C. Ma, D. Lu, Synthesis and anticancer activities of novel 8azapurine carbocyclic nucleoside hydrazones, Bioorg. Med. Chem. Lett 25 (2015) 4461-4463.

[14] Z.H. Li, D.X. Yang, P.F. Geng, J. Zhang, H.M. Wei, B. Hu, Q. Guo, X.H. Zhang, W.G. Guo, B. Zhao, B.B. Yu, L.Y. Ma, H.M. Liu, Design, synthesis and biological evaluation of $[1,2,3]$ triazolo[4,5-d]pyrimidine derivatives possessing a hydrazone moiety as antiproliferative agents, Eur. J. Med. Chem. 124 (2016) 967-980.

[15] Z.H. Li, X.Q. Liu, T.Q. Zhao, P.F. Geng, W.G. Guo, B. Yu, H.M. Liu, Design, synthesis and preliminary biological evaluation of new $[1,2,3]$ triazolo[4,5-d]pyrimidine/thiourea hybrids as antiproliferative agents, Eur. J. Med. Chem. 139 (2017) 741-749.

[16] P.F. Geng, X.O. Liu, T.O. Zhao, C.C. Wang Z.H. Li, J. Zhang, H.M. Wei, B. Hu, L.Y. Ma, H.M. Liu, Design, synthesis and in vitro biological evaluation of novel $[1,2,3]$ triazolo[4,5-d]pyrimidine derivatives containing a thiosemicarbazide moiety, Eur. J. Med. Chem. 146 (2018) 147-156.

[17] Z. Li, XO Liu, P.F. Geng FZ Suo, J.L Ma, B. Yu, T. Z Zhao, Z O Zhou, C.X. Huang, Y.C. Zheng, H.M. Liu, Discovery of $[1,2,3]$ triazolo[4,5-d]pyrimidine derivatives as novel LSD1 inhibitors, ACS Med. Chem. Lett. 8 (2017) 384-389.

[18] Q. Fan, Y. Wang, H. Yan, An NMR and DFT investigation on the interconversion of 9 -substituented- $\mathrm{N}^{6}$-hydrazone-8-azaadenine derivatives: proton migration or conformational isomerization? Struct. Chem. 29 (2018) 871-879.

[19] A. Buchanan, P. Newton, S. Pehrsson, T. Inghardt, T. Antonsson, P. Svensson, T. Sjögren, L. Öster, A. Janefeldt, A.S. Sandinge, F. Keyes, M. Austin, J. Spooner, P. Gennemark, M. Penney, G. Howells, T. Vaughan, S. Nylander, Structural and functional characterization of a specific antidote for ticagrelor, Blood 125 (2015) 3484-3490.

[20] J. Bojarska, M. Remko, A. Fruzinski, W. Maniukiewicz, The experimental and theoretical landscape of a new antiplatelet drug ticagrelor: insight into supramolecular architecture directed by $\mathrm{C}-\mathrm{H} \cdots \mathrm{F}, \pi \cdots \pi$ and $\mathrm{C}-\mathrm{H} \cdots \pi$ interactions, J. Mol. Struct. 1154 (2017) 290-300.

[21] M. Inam, J. Wu, J. Shen, C.U. Phan, G. Tang, X. Hu, Preparation and characterization of novel pharmaceutical co-crystals: ticagrelor with nicotinamide, Crystals 8 (2018) 336.

[22] Y. Li, C. Landqvist, S.W. Grimm, Disposition and metabolism of ticagrelor, a novel $\mathrm{P}_{2} \mathrm{Y}_{12}$ receptor antagonist, in mice, rats, and marmosets, Drug Metab. Dispos. 39 (2011) 1555-1567. 